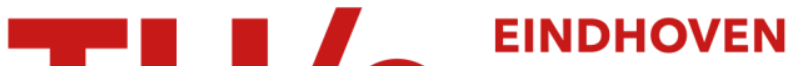

\section{Convergence of critical oriented percolation to super-Brownian motion above 4+1 dimensions}

\section{Citation for published version (APA):}

Hofstad, van der, R. W., \& Slade, G. (2003). Convergence of critical oriented percolation to super-Brownian motion above 4+1 dimensions. Annales de l'institut Henri Poincare (B): Probability and Statistics, 39(3), 413-485. https://doi.org/10.1016/S0246-0203(03)00008-6

DOI:

10.1016/S0246-0203(03)00008-6

Document status and date:

Published: 01/01/2003

\section{Document Version:}

Publisher's PDF, also known as Version of Record (includes final page, issue and volume numbers)

\section{Please check the document version of this publication:}

- A submitted manuscript is the version of the article upon submission and before peer-review. There can be important differences between the submitted version and the official published version of record. People interested in the research are advised to contact the author for the final version of the publication, or visit the $\mathrm{DOI}$ to the publisher's website.

- The final author version and the galley proof are versions of the publication after peer review.

- The final published version features the final layout of the paper including the volume, issue and page numbers.

Link to publication

\section{General rights}

Copyright and moral rights for the publications made accessible in the public portal are retained by the authors and/or other copyright owners and it is a condition of accessing publications that users recognise and abide by the legal requirements associated with these rights.

- Users may download and print one copy of any publication from the public portal for the purpose of private study or research.

- You may not further distribute the material or use it for any profit-making activity or commercial gain

- You may freely distribute the URL identifying the publication in the public portal.

If the publication is distributed under the terms of Article $25 \mathrm{fa}$ of the Dutch Copyright Act, indicated by the "Taverne" license above, please follow below link for the End User Agreement:

www.tue.nl/taverne

\section{Take down policy}

If you believe that this document breaches copyright please contact us at:

openaccess@tue.nl

providing details and we will investigate your claim. 


\title{
CONVERGENCE OF CRITICAL ORIENTED PERCOLATION TO SUPER-BROWNIAN MOTION ABOVE $4+1$ DIMENSIONS
}

\section{CONVERGENCE DE LA PERCOLATION ORIENTÉE CRITIQUE VERS LE SUPER MOUVEMENT BROWNIEN EN DIMENSION SUPÉRIEURE À $4+1$}

\author{
Remco VAN DER HOFSTAD ${ }^{\mathrm{a}, 1}$, Gordon SLADE $^{\mathrm{b}, *}$ \\ a Stieltjes Institute for Mathematics, Delft University, Mekelweg 4, 2628 CD Delft, The Netherlands \\ ${ }^{\mathrm{b}}$ Department of Mathematics, University of British Columbia, Vancouver, BC V6T 1Z2, Canada
}

Received 5 September 2001

ABSTRACT. - We consider oriented bond percolation on $\mathbb{Z}^{d} \times \mathbb{N}$, at the critical occupation density $p_{c}$, for $d>4$. The model is a "spread-out" model having long range parameterised by $L$. We consider configurations in which the cluster of the origin survives to time $n$, and scale space by $n^{1 / 2}$. We prove that for $L$ sufficiently large all the moment measures converge, as $n \rightarrow \infty$, to those of the canonical measure of super-Brownian motion. This extends a previous result of Nguyen and Yang, who proved Gaussian behaviour for the critical two-point function, to all $r$-point functions $(r \geqslant 2)$. We use lace expansion methods for the two-point function, and prove convergence of the expansion using a general inductive method that we developed in a previous paper. For the $r$-point functions with $r \geqslant 3$, we use a new expansion method.

(c) 2003 Éditions scientifiques et médicales Elsevier SAS

MSC: 60K35; 82B43

RÉSUMÉ. - On considére un modèle de percolation orientée sur les liens de $\mathbb{Z}^{d} \times \mathbb{N}$ à la densité d'occupation critique $p_{c}$, pour $d>4$. Le modèle comporte un effet de dispersion à longue portée paramétré par une longueur $L$. On considère les configurations dans lesquelles l'amas comprenant l'origine survit jusqu' au temps $n$, et on rééchelonne l'espace par un facteur $n^{1 / 2}$. On montre, pour $L$ assez grand, la convergence de tous les moments à valeurs mesures vers ceux du super mouvement brownien. On étend ainsi un résultat préalablement obtenu par Nguyen et Yang, qui ont montré le comportement gaussien de la fonction à deux points critique, au cas des fonctions à $r$ points. La convergence du développement de la fonction à deux points est établie à l'aide d'une méthode générale d'induction développée dans un article précédent. Une nouvelle méthode de développement est employée pour $r$ supérieur à trois.

(c) 2003 Éditions scientifiques et médicales Elsevier SAS

\footnotetext{
* Corresponding author.

E-mail addresses: rhofstad@ win.tue.nl (R.van der Hofstad), slade@ math.ubc.ca (G. Slade).

${ }^{1}$ Present address: Department of Mathematics and Computer Science, Eindhoven University of Technology, P.O. Box 513, 5600 MB Eindhoven, The Netherlands.
} 


\section{Introduction and results}

\subsection{Introduction}

The lace expansion has been used to prove mean-field behaviour in high dimensions for models of self-avoiding walks, lattice trees and lattice animals, and percolation. In particular, there has been recent progress in identifying the scaling limit of lattice trees above eight dimensions, and of the incipient infinite percolation cluster above six dimensions, as integrated super-Brownian excursion (ISE) [12,13,22,23,37]. In this paper we prove a related result for oriented percolation (also called directed percolation).

We consider "spread-out" oriented bond percolation on the lattice $\mathbb{Z}^{d} \times \mathbb{N}$, at the critical bond occupation density $p=p_{c}$, with $d>4$. As we will explain in more detail below, the spread-out model involves a parameter $L \geqslant 1$ that describes the extent to which connections in the model are spread-out in space. We study configurations in which the cluster of the origin survives to time $n$, and scale space by $n^{1 / 2}$. We prove that for $L$ sufficiently large, all oriented percolation moment measures converge to the moment measures of the canonical measure of super-Brownian motion, in the limit $n \rightarrow \infty$. This result goes part way to proving that the scaling limit of the incipient infinite cluster is the canonical measure of super-Brownian motion, for $d>4$. An additional tightness result, which we have not proved, would be required to conclude weak convergence in the sense of measure-valued processes.

The spread-out models are believed to lie in the same universality class as the nearestneighbour model, for all finite $L \geqslant 1$. Our results therefore support the conjecture that the scaling limit of critical oriented percolation is super-Brownian motion, for the nearestneighbour model with $d+1>5$. We believe it should be possible to extend our results to the nearest-neighbour model for sufficiently high dimensions $d$, but this work has not been carried out.

The limit $L \rightarrow \infty$ is a mean-field limit, and our method employs the lace expansion to perturb about a mean-field theory. However, we do keep $L$ finite, and our results include models with long but finite range. This should be contrasted with the recent work of Durrett and Perkins [14] (see also [10]), who prove that the critical contact process converges to super-Brownian motion in dimensions $d \geqslant 2$, for models in which the range of infection diverges at a particular rate as time goes to infinity. In their limit, mean-field behaviour is observed also below $d=4$. It would be of interest to extend our results for oriented percolation to the finite range contact process for $d>4$. The recent proof of the triangle condition for the contact process with $d>4$ and $L$ sufficiently large [35] would provide a starting point for such an extension.

The identification of $d+1=5$ as the upper critical dimension for oriented percolation originated in the physical analysis of [33]. Recently, hyperscaling inequalities for oriented percolation and the contact process have been derived and used to show that mean-field critical behaviour is incompatible with $d+1<5$ [36].

\subsection{Main results}

The spread-out oriented percolation models are defined as follows. Consider the graph with vertices $\mathbb{Z}^{d} \times \mathbb{N}$ and directed bonds $((x, n),(y, n+1))$, for $n \geqslant 0$ and $x, y \in \mathbb{Z}^{d}$. 
Let $D$ be a fixed function $D: \mathbb{Z}^{d} \rightarrow[0,1]$ which is symmetric under replacement of any component $x_{i}$ of $x \in \mathbb{Z}^{d}$ by $-x_{i}$, and under permutation of the components of $x$. Let $p \in\left[0,\|D\|_{\infty}^{-1}\right]$, where $\|\cdot\|_{\infty}$ denotes the supremum norm, so that $p D(y-x) \leqslant 1$. We associate to each directed bond $((x, n),(y, n+1))$ an independent random variable taking the value 1 with probability $p D(y-x)$ and 0 with probability $1-p D(y-x)$. We say a bond is occupied when the corresponding random variable takes the value 1 , and vacant when the random variable is 0 . The joint probability distribution of the bond variables will be denoted $\mathbb{P}_{p}$, with corresponding expectation denoted $\mathbb{E}_{p}$. Note that $p$ is not a probability.

We say that $(x, n)$ is connected to $(y, m)$, and write $(x, n) \rightarrow(y, m)$, if there is an oriented path from $(x, n)$ to $(y, m)$ consisting of occupied bonds. Note that this is only possible when $m \geqslant n$. We write $C(x, n)$ for the set of sites $(y, m)$ such that $(x, n) \rightarrow$ $(y, m)$, and denote the cardinality of $C(x, n)$ by $|C(x, n)|$. We adopt the convention that every site is connected to itself, so that $(x, n) \in C(x, n)$ for every site $(x, n)$. Define $p_{c}$ to be the supremum of the set of $p \in\left[0,\|D\|_{\infty}^{-1}\right]$ for which $\mathbb{E}_{p}|C(0,0)|<\infty$. The critical value $p_{c}$ can also be characterised as the infimum of the set of $p$ for which $|C(0,0)|$ is infinite with positive probability $[2,30]$.

The function $D$ will always be assumed to obey the properties of Assumption D of [27]. Assumption D involves a positive parameter $L$, which serves to spread out the connections, and which we will take to be large. The parameterisation has been chosen in such a way that $p_{c}$ will be asymptotically equal to 1 as $L \rightarrow \infty$.

For an absolutely summable function $f: \mathbb{Z}^{(r-1) d} \rightarrow \mathbb{C}(r=2,3, \ldots)$ and for $\vec{k}=$ $\left(k_{1}, \ldots, k_{r-1}\right)$ with each $k_{j} \in[-\pi, \pi]^{d}$, we define the Fourier transform

$$
\hat{f}(\vec{k})=\sum_{y_{1}, \ldots, y_{r-1} \in \mathbb{Z}^{d}} f(\vec{y}) \mathrm{e}^{i \vec{k} \cdot \vec{y}},
$$

where $\vec{k} \cdot \vec{y}=\sum_{j=1}^{r-1} k_{j} \cdot y_{j}$. When $r=2$, we write simply $k$ in place of $\vec{k}$.

The properties of Assumption D are as follows. We require that $\sum_{x \in \mathbb{Z}^{d}} D(x)=1$, that $D(x) \leqslant C L^{-d}$ uniformly in $x$, and that there is an $\varepsilon>0$ such that $\sum_{x}|x|^{2+2 \varepsilon} D(x)<\infty$. In this paper, we strengthen the latter to require that

$$
\sup _{x}|x|^{2} D(x) \leqslant C L^{2-d} \text { and } \quad \sum_{x}|x|^{2+2 \varepsilon} D(x) \leqslant C L^{2+2 \varepsilon} .
$$

Let

$$
\sigma^{2}=\sum_{x \in \mathbb{Z}^{d}}|x|^{2} D(x)
$$

where $|\cdot|$ denotes the Euclidean norm on $\mathbb{R}^{d}$. We also require that there are positive constants $\eta, c_{1}, c_{2}$ such that

$$
\begin{array}{rlrl}
c_{1} L^{2}|k|^{2} \leqslant 1 & -\widehat{D}(k) \leqslant c_{2} L^{2}|k|^{2} & & \left(\|k\|_{\infty} \leqslant L^{-1}\right), \\
1-\widehat{D}(k)>\eta & & \left(\|k\|_{\infty} \geqslant L^{-1}\right), \\
1-\widehat{D}(k)<2-\eta & & \left(k \in[-\pi, \pi]^{d}\right) .
\end{array}
$$


It follows from (1.4) that $\sigma$ is bounded above and below by multiples of $L$.

Examples of functions $D$ obeying the above assumptions are given in [27]. A simple example is

$$
D(x)= \begin{cases}(2 L+1)^{-d} & \|x\|_{\infty} \leqslant L, \\ 0 & \text { otherwise. }\end{cases}
$$

In this example, the bonds are given by $((x, n),(y, n+1))$ with $\|x-y\|_{\infty} \leqslant L$, and a bond is occupied with probability $p(2 L+1)^{-d}$.

We begin by stating our results for the two-point function.

\subsubsection{The two-point function}

Given $D$ obeying the above assumptions, $p \in\left[0,\|D\|_{\infty}^{-1}\right], n \geqslant 0$ and $x \in \mathbb{Z}^{d}$, we define the two-point function

$$
\tau_{n}(x)=\mathbb{P}_{p}((0,0) \rightarrow(x, n)) .
$$

Our main result for the two-point function is the following theorem. In its statement, $\varepsilon$ is the parameter in the tail estimate assumed for $D$, and $\sigma$ is given by (1.3).

THEOREM 1.1. - Let $d>4, p=p_{c}$, and $\delta \in\left(0,1 \wedge \varepsilon \wedge \frac{d-4}{2}\right)$. There is an $L_{0}=L_{0}(d)$ such that for $L \geqslant L_{0}$ there exist positive constants $v$ and $A$ (depending on $d$ and $L$ ), and $C_{1}, C_{2}$ (depending only on $d$ ), such that the following statements hold as $n \rightarrow \infty$ :

(a)

$$
\hat{\tau}_{n}\left(k / \sqrt{v \sigma^{2} n}\right)=A \mathrm{e}^{-\frac{|k|^{2}}{2 d}}\left[1+\mathcal{O}\left(|k|^{2} n^{-\delta}\right)+\mathcal{O}\left(n^{-(d-4) / 2}\right)\right],
$$

(b)

$$
\frac{1}{\hat{\tau}_{n}(0)} \sum_{x}|x|^{2} \tau_{n}(x)=v \sigma^{2} n\left[1+\mathcal{O}\left(n^{-\delta}\right)\right]
$$

(c)

$$
C_{1} L^{-d} n^{-d / 2} \leqslant \sup _{x \in \mathbb{Z}^{d}} \tau_{n}(x) \leqslant C_{2} L^{-d} n^{-d / 2},
$$

with the error estimate in (a) uniform in $k \in \mathbb{R}^{d}$ with $|k|^{2}(\log n)^{-1}$ sufficiently small.

Constants implied by the $\mathcal{O}$ notation in the above error terms may depend on $L$. Parts (a) and (b) of Theorem 1.1 were first proved by Nguyen and Yang [32] using generating function methods, with somewhat weaker error estimates. Our proof uses very different methods, based on induction in $n$ rather than generating functions. Part (c) is new, and will be essential in our analysis of the $r$-point functions for $r \geqslant 3$.

Our proof makes use of the general inductive method of [27]. The inductive method requires the verification of certain assumptions, which we will verify in this paper. Once these assumptions have been verified, a number of further consequences follow immediately from [27]. In particular, it follows that

$$
p_{c}=1+\mathcal{O}\left(L^{-d}\right), \quad A=1+\mathcal{O}\left(L^{-d}\right), \quad v=1+\mathcal{O}\left(L^{-d}\right)
$$


where the constants in the error terms here of course do not depend on $L$. Identities obeyed by $p_{c}, A$ and $V$ are given in (2.11)-(2.13) below. As is explained in [27], a version of a local central limit theorem for $\tau_{n}(x)$ also follows. (A new approach to the lace expansion, based on the Banach fixed point theorem, has been recently introduced [7]. An extension of this approach to oriented percolation might possibly lead to an improved local central limit theorem.)

In addition, an infrared bound follows from the induction hypotheses of [27], once we verify the necessary assumptions. To state the infrared bound, we define

$$
T_{z}(k)=\sum_{n=0}^{\infty} \hat{\tau}_{n}(k) z^{n} \quad(z \in[0,1)) .
$$

It is then possible to show, using the induction hypotheses of [27], that under the assumptions of Theorem 1.1 it follows that

$$
\left|T_{z}(k)\right| \leqslant \frac{C}{|k|^{2}+(1-z)}
$$

uniformly in $k \in[-\pi, \pi]^{d}, z \in[0,1), p \leqslant p_{c}$, for some constant $C$ (depending on $L$ ). The infrared bound (1.14) played a crucial role in the analysis of $[31,32]$. In particular, (1.14) implies the triangle condition of [3], which can also be derived directly from Theorem 1.1(a, c).

For $k=0$, the Fourier transform $\hat{\tau}_{n}(0)$ is given by

$$
\hat{\tau}_{n}(0)=\sum_{x \in \mathbb{Z}^{d}} \tau_{n}(x)=\mathbb{E}_{p}\left[\sum_{x \in \mathbb{Z}^{d}} I[(0,0) \rightarrow(x, n)]\right]=\mathbb{E}_{p}\left|C(0,0) \cap\left(\mathbb{Z}^{d} \times\{n\}\right)\right| .
$$

Theorem 1.1(a) shows that this expectation converges to a nonzero finite constant $A$ as $n \rightarrow \infty$, when $p=p_{c}$. For $p<p_{c}$ in general dimensions, the corresponding limit is zero, while for $p>p_{c}$ it is infinite. (See [2,3] or [30] for the relevant exponential decay when $p<p_{c}$, and $[6,19]$ for the relevant shape theorem when $p>p_{c}$.)

\subsubsection{The $r$-point functions, $r \geqslant 3$}

The $r$-point functions, for all $r \geqslant 2$, are defined by

$$
\tau_{n_{1}, \ldots, n_{r-1}}^{(r)}\left(x_{1}, \ldots, x_{r-1}\right)=\mathbb{P}_{p}\left((0,0) \rightarrow\left(x_{i}, n_{i}\right) \text { for each } i=1, \ldots, r-1\right) .
$$

Note that the event on the right side makes no statement about the occurrence of $\left(x_{i}, n_{i}\right) \rightarrow\left(x_{j}, n_{j}\right)$ for any $i \neq j$.

In order to state our result for the $r$-point functions, we require the notion of shape. Shapes are certain rooted binary trees. For $r \geqslant 2$, we give a recursive definition of the set $\Sigma_{r}$ of $r$-shapes, as follows. Each $r$-shape has $2 r-3$ edges, $r-2$ vertices of degree 3 (the branch points) and $r$ vertices of degree 1 (the leaves) labelled $0,1, \ldots, r-1$. There is a unique 2 -shape given by the tree consisting of vertex 0 joined by a single edge to vertex 1 . We think of this shape as corresponding to a particle travelling from vertex 0 to vertex 1 . There is a unique 3 -shape, consisting of three vertices $0,1,2$ each joined 

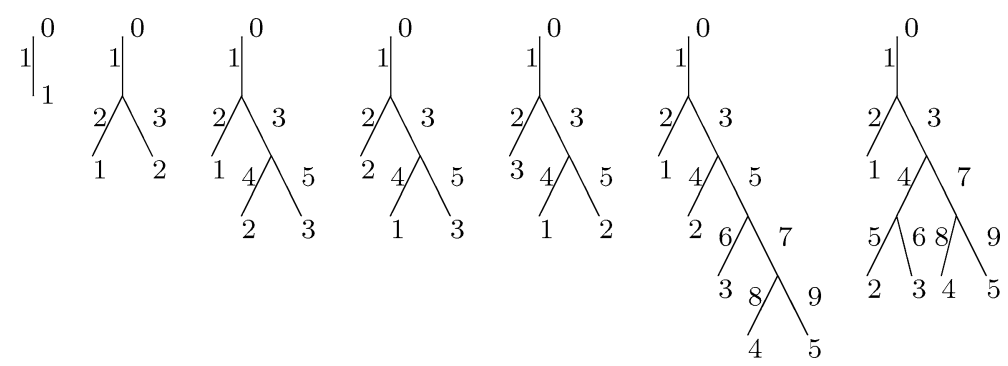

Fig. 1. The shapes for $r=2,3,4$, and examples of the $7 \cdot 5 \cdot 3=105$ shapes for $r=6$. The shapes' edge labellings are arbitrary but fixed.

by an edge to a fourth (unlabelled) vertex. We think of this shape as corresponding to a particle that originates at 0 , then splits after some time into two particles that travel to 1 and 2. In general, for $r \geqslant 3$, to each $(r-1)$-shape $\alpha$, we obtain $2 r-5 r$-shapes by chosing one of the $2 r-5$ edges of $\alpha$, adding a vertex on that edge together with a new edge that joins the added vertex to a new leaf $r-1$. The resulting $r$-shapes represent the different ways in which an additional $r$ th particle can be added to the family tree of $r-1$ particles represented by $\alpha$. Thus there is a unique shape for $r=2$ and $r=3$, and $\prod_{j=3}^{r}(2 j-5)$ distinct shapes for $r \geqslant 4$. When $r$ is clear from the context, we will refer to an $r$-shape simply as a shape. For notational convenience, we associate to each shape an arbitrary labelling of its $2 r-3$ edges, with labels $1, \ldots, 2 r-3$. This arbitrary choice of edge labelling is fixed once and for all; see Fig. 1.

We will often consider vectors with $r-1$ components, as in (1.16), as well as vectors indexed by the edges of a shape, with $2 r-3$ components. To distinguish $(r-1)$-component vectors from $(2 r-3)$-component vectors, we will write, e.g., $\vec{n}=\left(n_{1}, \ldots, n_{r-1}\right)$, whereas $\bar{m}=\left(m_{1}, \ldots, m_{2 r-3}\right)$. Given a shape $\alpha \in \Sigma_{r}$ and $\vec{k}=$ $\left(k_{1} \ldots, k_{r-1}\right) \in \mathbb{R}^{(r-1) d}$, we introduce $\bar{k}(\alpha) \in \mathbb{R}^{(2 r-3) d}$ as follows. First, for each vertex $j$ of degree 1 in $\alpha$, other than vertex 0 , let $\omega_{j}$ be the set of edges in $\alpha$ on the path from 0 to $j(j=1, \ldots, r-1)$. For $\ell=1, \ldots, 2 r-3$, we define the $\ell$ th component $\bar{k}_{\ell}(\alpha) \in \mathbb{R}^{d}$ of $\bar{k}(\alpha)$ by

$$
\bar{k}_{\ell}(\alpha)=\sum_{j=1}^{r-1} k_{j} I\left[\ell \in \omega_{j}\right],
$$

where, on the right side, $k_{j}$ denotes the $j$ th component of $\vec{k}$ and $I$ is an indicator function. Conversely, given $\bar{s} \in \mathbb{R}_{+}^{2 r-3}$, we define the $j$ th component $\vec{s}_{j}(\alpha)$ of $\vec{s}(\alpha) \in \mathbb{R}_{+}^{r-1}$ by

$$
\vec{s}_{j}(\alpha)=\sum_{\ell \in \omega_{j}} s_{\ell} .
$$

We also define an $(r-2)$-dimensional subset $R_{\vec{t}}(\alpha)$ of $\mathbb{R}_{+}^{2 r-3}$ by

$$
R_{\vec{t}}(\alpha)=\{\bar{s}: \vec{s}(\alpha)=\vec{t}\} .
$$

For example, for $r=3$, there is a unique shape $\alpha$ and we have simply

$$
R_{\vec{t}}(\alpha)=\left\{\left(s, t_{1}-s, t_{2}-s\right): s \in\left[0, t_{1} \wedge t_{2}\right]\right\} .
$$


We will abuse notation by writing $\int_{R_{\vec{t}}(\alpha)} \mathrm{d} \bar{s}$ for the $(r-2)$-dimensional integral over $R_{\vec{t}}(\alpha)$.

Our main result for the $r$-point functions is the following theorem. In its statement, the constants $A$ and $v$ are the same as those appearing in Theorem 1.1.

THEOREM 1.2. - Let $d>4, p=p_{c}, \delta \in\left(0,1 \wedge \varepsilon \wedge \frac{d-4}{2}\right), r \geqslant 3, \vec{t}=\left(t_{1}, \ldots, t_{r-1}\right) \in$ $(0, \infty)^{r-1}$, and $\vec{k}=\left(k_{1}, \ldots, k_{r-1}\right) \in \mathbb{R}^{(r-1) d}$. There is a constant $V$, with $|V-1| \leqslant$ $C L^{-d}$, and an $L_{0}=L_{0}(d)$ (independent of $r$ ) such that for $L \geqslant L_{0}$,

$$
\hat{\tau}_{\lfloor n \vec{t}\rfloor}^{(r)}\left(\vec{k} / \sqrt{v \sigma^{2} n}\right)=n^{r-2} V^{r-2} A^{2 r-3}\left[\sum_{\alpha \in \Sigma_{r}} \int_{R_{\vec{t}^{\prime}}(\alpha)} \mathrm{d} \bar{s} \prod_{\ell=1}^{2 r-3} \mathrm{e}^{-\left|\bar{k}_{\ell}(\alpha)\right|^{2} \bar{s}_{\ell} / 2 d}+\mathcal{O}\left(n^{-\delta}\right)\right],
$$

with the error estimate uniform in $\vec{k}$ in a bounded subset of $\mathbb{R}^{(r-1) d}$.

Constants implied by the $\mathcal{O}$ notation can depend on $L$ and on the $t_{\ell}$. Uniformity cannot be expected as $t_{\ell} \rightarrow 0$, since taking $t_{\ell}=0$ amounts to a reduction in $r$ and changes the branching structure.

For $r=3$, (1.21) reduces to

$$
\begin{aligned}
& \hat{\tau}_{\lfloor n \vec{t}\rfloor}^{(3)}\left(\vec{k} / \sqrt{v \sigma^{2} n}\right) \\
& \quad=n V A^{3}\left[\int_{0}^{t_{1} \wedge t_{2}} \mathrm{~d} s \mathrm{e}^{-\left|k_{1}+k_{2}\right|^{2} s / 2 d} \mathrm{e}^{-\left|k_{1}\right|^{2}\left(t_{1}-s\right) / 2 d} \mathrm{e}^{-\left|k_{2}\right|^{2}\left(t_{2}-s\right) / 2 d}+\mathcal{O}\left(n^{-\delta}\right)\right] .
\end{aligned}
$$

Eq. (1.22) can be interpreted as indicating that a cluster connecting the origin to $\left(x_{1},\left\lfloor n t_{1}\right\rfloor\right)$ and $\left(x_{2},\left\lfloor n t_{2}\right\rfloor\right)$, with the $x_{i}$ of order $n^{1 / 2}$, can be considered to decompose into a product of three independent two-point functions joined together at a branch point. Each two-point function gives rise to a Gaussian, together with a factor $A$, according to Theorem 1.1(a). This decomposition into independent two-point functions is not exact, but is compensated by the vertex factor $V$ associated with the branch point. The integral with respect to $s$ corresponds to a sum over possible temporal locations of the branch point, with the additional factor $n$ accounting for the change from a sum to an integral.

Similar considerations apply to (1.21), with additional structure due to the proliferation of shapes. There are $r-2$ branch points in the general case, each contributing $n V$, and $2 r-3$ two-point functions, each contributing $A$ times a Gaussian. The integral over $R_{\vec{t}}(\alpha)$ corresponds to a sum over time intervals between the various branch points, and is constrained so that the shape's leaves are specified by the times $\lfloor n \vec{t}\rfloor$.

It is an elementary consequence of the tree graph bounds [3,18], together with the bound on the two-point function of Theorem 1.1(a), that the left side of (1.21) is bounded above by a multiple of $n^{r-2}$. By (1.21), this elementary upper bound gives the correct power of $n$, above the upper critical dimension.

\subsubsection{Convergence to super-Brownian motion}

Theorems 1.1 and 1.2 can be rephrased to say that, under their hypotheses, the moment measures of rescaled critical oriented percolation converge to those of the canonical measure of super-Brownian motion. We now make this interpretation of Theorems 1.1 and 1.2 more explicit. Throughout this section, we write $l=r-1 \in\{1,2, \ldots\}$. 
First, for $t \in(0, \infty)$, we define $X_{n, t}$ as the discrete finite random measure on $\mathbb{R}^{d} \times \mathbb{N}$ giving mass $\left(A^{2} V n\right)^{-1}$ at each site in $\left(v \sigma^{2} n\right)^{-1 / 2} C(0,0)$. For $\vec{t} \in(0, \infty)^{l}$, the characteristic function of the $l$ th moment measure of this random measure is given by

$$
\widehat{M}_{n, \vec{t}}^{(l)}(\vec{k})=\left(A^{2} V n\right)^{-l} \hat{\tau}_{\lfloor n \vec{t}\rfloor}^{(l+1)}\left(\vec{k} / \sqrt{v \sigma^{2} n}\right) .
$$

We want to compare $\widehat{M}_{n, \vec{t}}^{(l)}(\vec{k})$ with the corresponding quantity for the canonical measure of super-Brownian motion.

Super-Brownian motion has been discussed in several recent books and major reviews [11,16,17,28,34], as a basic example of a measure-valued Markov process. In particular, the canonical measure of super-Brownian motion is described in $[28,34]$. See also [38] for a nontechnical introduction. The canonical measure for super-Brownian motion is the scaling limit of a single critical branching random walk which starts at the origin and survives for some positive rescaled time. Since critical branching random walk survives to time $n$ with probability proportional to $n^{-1}$, to obtain a nontrivial limit it is necessary to multiply probabilities by a factor $n$. This produces an unnormalised measure, the canonical measure, in the scaling limit. The canonical measure is a measure $\mathbb{N}_{0}$ on continuous paths from $[0, \infty)$ into non-negative finite measures on $\mathbb{R}^{d}$. We take $\mathbb{N}_{0}$ to be normalised to have unit branching and diffusion rates.

By definition, the $l$ th moment measure of $\mathbb{N}_{0}$ has Fourier transform

$$
\widehat{M}_{\vec{t}}^{(l)}(\vec{k})=\mathbb{N}_{0}\left(\int_{\mathbb{R}^{d l}} X_{t_{1}}\left(\mathrm{~d} x_{1}\right) \ldots X_{t_{l}}\left(\mathrm{~d} x_{l}\right) \prod_{j=1}^{l} \mathrm{e}^{i k_{j} \cdot x_{j}}\right)
$$

where each $X_{t}$ is a random non-negative finite measure on $\mathbb{R}^{d}$. Using the notation of (1.17)-(1.19), $\widehat{M}_{\vec{t}}^{(l)}(\vec{k})$ is given by

$$
\widehat{M}_{\vec{t}}^{(l)}(\vec{k})= \begin{cases}\mathrm{e}^{-|k|^{2} t / 2 d} & (l=1), \\ \sum_{\alpha \in \Sigma_{l+1}} \int_{R_{\vec{t}}(\alpha)} \mathrm{d} \bar{s} \prod_{j=1}^{2 l-1} \mathrm{e}^{-\left|k_{j}(\alpha)\right|^{2} \bar{s}_{j} / 2 d} & (l \geqslant 2) .\end{cases}
$$

Formula (1.25) is essentially [1, Theorem 3.1] (see also [15] and [28, Proposition IV.2(ii)]). The following corollary then follows immediately from Theorems 1.1 and 1.2. It shows that the moment measures of $\mathbb{N}_{0}$ provide the joint mass distributions, at distinct times, of the average over configurations of the scaling limit of spread-out critical oriented percolation above dimensions $4+1$.

COROLlary 1.3. - Let $d>4, p=p_{c}, l \geqslant 1, t_{i} \in(0, \infty)(i=1, \ldots, l), \vec{t}=$ $\left(t_{1}, \ldots, t_{l}\right)$, and $\vec{k}=\left(k_{1}, \ldots, k_{l}\right) \in \mathbb{R}^{d l}$. There is an $L_{0}=L_{0}(d)$ such that for $L \geqslant L_{0}$,

$$
\lim _{n \rightarrow \infty} A V n \widehat{M}_{n, \vec{t}}^{(l)}(\vec{k})=\widehat{M}_{\vec{t}}^{(l)}(\vec{k})
$$

In other words, $A V n \mathbb{P}\left(\left\{X_{n, t}\right\}_{t>0} \in \cdot\right)$ converges to $\mathbb{N}_{0}$ in the sense of convergence of finite-dimensional distributions. 
Corollary 1.3 can be interpreted as stating that spread-out critical oriented percolation and critical branching random walk have the same scaling limit, for $d>4$ (compare [34, Theorem II.7.3(a)]). A crucial difference between oriented percolation and branching random walk is that particles can coexist at the same site for the latter, but not for the former.

Corollary 1.3 is a statement of convergence of finite-dimensional distributions. To prove weak convergence, as a measure-valued process, of rescaled spread-out oriented percolation for $d>4$ to the canonical measure of super-Brownian motion, it would be necessary also to prove tightness. We do not address tightness in this paper. Another problem not addressed here is to prove that there is a constant $B$ such that critical oriented percolation survives to time $n$ with probability asymptotic to $(B n)^{-1}$, for $d>4$. It is shown in [24, Theorem 1.5] that if the survival probability is in fact asymptotic to $(B n)^{-1}$ then it must be the case that $B=A V / 2$, which in turn implies that the factor $A V n$ in (1.26) corresponds asymptotically to twice the reciprocal of the survival probability.

Our results are restricted to dimensions above the upper critical dimension $4+1$, below which different scaling behaviour is expected. Often the upper critical dimension of a statistical mechanical model can be understood as the dimension above which particular random objects generically do not intersect. For example, the critical dimension of self-avoiding walk is $d=4$, which can be understood as the dimension above which two 2-dimensional Brownian motion paths typically do not intersect. For non-oriented percolation, the critical dimension is $d=6$, which can be understood as the dimension above which a 4-dimensional cluster does not intersect a 2-dimensional backbone [22].

For oriented percolation, as we will discuss in more detail in Section 3, the upper critical dimension can be understood as the dimension above which the graphs of Brownian motion and super-Brownian motion do not intersect. Intersection of the graphs implies a collision of the two processes at the same time. It is known that $d=4$ is critical for such a collision [5]. This can be understood heuristically in the following way. We first assume that since both processes are moving, we may think of one as being stationary (this is a leap of faith). Regarding the super-Brownian motion as stationary, its support at fixed time is 2-dimensional. The Brownian path, which is two-dimensional, will generically not hit this support in dimensions greater than $4=2+2$. Alternately, if we regard the Brownian motion as being fixed, then its support is a point, hence 0 -dimensional. The 4-dimensional range of super-Brownian motion will generically not hit this point in dimensions above $4=4+0$.

Oriented percolation has no infinite cluster at the critical point [6,19]. The notion of incipient infinite cluster is used to refer to the large emerging structures that are nevertheless present at the critical point. In [24], a construction of the incipient infinite cluster is given for spread-out oriented percolation above $4+1$ dimensions. We conjecture that the scaling limit of the incipient infinite cluster for oriented percolation in dimensions $d+1>4+1$ is the canonical measure of super-Brownian motion, conditioned on survival for all time. 


\subsection{Scaling limits and super-Brownian motion}

Recently, super-Brownian motion has been shown to arise in scaling limits of a number of models in statistical mechanics and interacting particle systems. We have already mentioned the work of [14] proving convergence of the critical contact process to super-Brownian motion for $d \geqslant 2$, in a particular limit in which the range of infection diverges to infinity with time. In addition, the finite-range voter model converges to super-Brownian motion in dimensions $d \geqslant 2$ [9]. These results for the contact process and voter model use methods quite different from ours, and are reviewed in [10].

Our methods are based on the lace expansion, which was first used to link scaling limits with super-processes in $[12,13]$. There it was shown that sufficiently spreadout lattice trees in dimensions $d>8$, or nearest-neighbour lattice trees in sufficiently high dimensions, converge to ISE (integrated super-Brownian excursion), as had been conjectured by Aldous [4]. ISE is the time integral of the canonical measure of superBrownian motion, conditioned to have total mass 1 . Later, in [22,23], results linking nonoriented percolation and ISE were obtained. The fact that super-Brownian motion arises in these diverse contexts involving critical branching demonstrates that super-Brownian motion has a universal character. In this section, we discuss the work on non-oriented percolation in more detail, and discuss natural conjectures for both oriented and nonoriented percolation.

\subsubsection{Non-oriented percolation and ISE}

The upper critical dimension for non-oriented percolation on $\mathbb{Z}^{d}$ is 6 . Consider $d>6$ and $p=p_{c}$, and condition on the event that the connected cluster of the origin consists of exactly $N$ sites. The work of [22,23] provides partial results supporting the hypothesis that the scaling limit of such a cluster, with space scaled by $N^{1 / 4}$, is ISE. However, in that work there is no explicit percolation "time" variable, and the results correspond to time having been "integrated out".

\subsubsection{Oriented percolation and ISE}

In our results for oriented percolation, we condition the cluster of the origin to reach time $n$, but do not condition on the total size of the cluster. It is natural to conjecture that if we condition the cluster of the origin to have size $N=n^{2}$, and scale time by $n$ and space by $n^{1 / 2}$, the scaling limit will be ISE. A formulation of the conjecture in terms of generating functions was given in [37]. It is an open problem to prove a result of this type linking critical oriented percolation and ISE, for $d>4$. We expect that the methods of $[22,23]$ could be extended to provide such a link.

\subsubsection{Non-oriented percolation and super-Brownian motion}

As mentioned above, the work of [22,23] on non-oriented percolation does not involve a time variable. It would be of interest to study the scaling limit of a cluster in critical non-oriented percolation in terms of a time variable, with or without fixing the total cluster size. Here we discuss the case analogous to our work on oriented percolation, in which the cluster size is not fixed.

To introduce a natural candidate for a time variable, we define the backbone $B(x)$ of a cluster containing 0 and $x \in \mathbb{Z}^{d}$ to be the set of sites $u \in \mathbb{Z}^{d}$ for which there are 
bond-disjoint connections between 0 and $u$, and between $u$ and $x$. We then think of the number of sites $|B(x)|$ in the backbone $B(x)$ as being a time variable analogous to the time variable $n$ in oriented percolation. Define

$$
t_{\vec{n}}^{(r)}(\vec{x})=\mathbb{P}_{p_{c}}\left(0 \rightarrow x_{i} \text { and }\left|B\left(x_{i}\right)\right|=n_{i} \text { for each } i=1, \ldots, r-1\right) .
$$

Then $t_{\vec{n}}^{(r)}(\vec{x})$ is analogous to the oriented percolation probability $\tau_{\vec{n}}^{(r)}(\vec{x})$ of $(1.16)$.

We conjecture that for non-oriented percolation (nearest-neighbour or spread-out) in dimensions $d>6$, the scaling limit of the cluster of the origin, in which backbones scale as $n$ and space scales as $n^{1 / 2}$, is super-Brownian motion. In particular, the conjecture includes the statement that Corollary 1.3 holds with $t_{\lfloor n \vec{t}\rfloor}^{(r)}$.eplacing $\tau_{\lfloor n \vec{t}\rfloor}^{(r)}$.

An alternate time variable would be the number of pivotal bonds for the connection between 0 and $x_{i}$. We expect this to scale in the same manner as the backbone size, for $d>6$, leading to the same scaling limit.

\subsection{Organisation}

The remainder of this paper is organised as follows. In Section 2, we give a detailed overview of the proof of Theorems 1.1 and 1.2. The proof is based on the lace expansion combined with the results of [27] pertaining to induction on $n$ for the two-point function, and on induction on $r$ for the $r$-point functions with $r \geqslant 3$. In Section 2, we reduce the proof of Theorems 1.1 and 1.2 to the estimation of several quantities arising in the lace expansion. These estimates are summarised in Propositions 2.2 and 2.3. Sections 3-7 are devoted to the proof of these two propositions.

In Section 3, we review the lace expansion method for the two-point function, which is the basis for the proof of Theorem 1.1. In Section 4, we obtain bounds on the expansion for the two-point function that verify the hypotheses of the induction method of [27], and complete the proof of Proposition 2.2 and Theorem 1.1.

To analyse the $r$-point functions for $r \geqslant 3$, we extend the expansion for the two-point function to general $r$-point functions in Section 5. The $r$-point functions, for $r \geqslant 3$, are then studied in Section 6 using a second expansion, as was done for non-oriented percolation in [22] and for lattice trees in [13,21]. The expansions used here for the $r$ point functions are simpler than the related expansions of $[22,23]$, as the magnetic field employed in $[22,23]$ is not used here.

Finally, in Section 7, we obtain bounds on quantities arising in expansions for the $r$-point functions, to prove Proposition 2.3 and complete the proof of Theorem 1.2.

\section{Overview and reduction of the proof}

In this section, we reduce the proof of Theorems 1.1 and 1.2 to Propositions 2.2 and 2.3. In the process, we provide an overview of the entire proof. Proposition 2.2 will be proved in Sections 3 and 4, and Proposition 2.3 will be proved in Sections 5-7. 


\subsection{The two-point function}

\subsubsection{The expansion}

The proof of Theorem 1.1 makes use of an expansion for the two-point function, which we state below. We postpone the derivation of the expansion to Section 3, and here we provide only a brief motivation. We will make use of the convolution of functions, which is defined for absolutely summable functions $f, g$ on $\mathbb{Z}^{d}$ by

$$
(f * g)(x)=\sum_{y} f(y) g(x-y) .
$$

To motivate the basic idea underlying the expansion, we consider the much simpler corresponding expansion for random walk. We will abuse notation by writing

$$
D((x, n))=D(x) \delta_{n, 1} .
$$

The two-point function for random walk is defined by setting $q_{0}(x)=\delta_{0, x}$ and

$$
q_{n}(x)=\sum_{\omega: 0 \rightarrow x} p^{n} \prod_{j=1}^{n} D(\omega(j)-\omega(j-1)) \quad(n \geqslant 1),
$$

where the sum is over all walks $\omega:\{0, \ldots, n\} \rightarrow \Lambda$ with $\omega(j) \in \mathbb{Z}^{d} \times\{j\}, \omega(0)=(0,0)$ and $\omega(n)=(x, n)$. To obtain an "expansion" for $q_{n}(x)$, we simply observe that by dividing the walk into two parts, consisting of its first step and the last $n-1$ steps, we obtain

$$
q_{n}(x)=p\left(D * q_{n-1}\right)(x) \quad(n \geqslant 1) .
$$

To adapt (2.4) to oriented percolation, we will regard an oriented percolation cluster connecting $(0,0)$ to $(x, n)$ as a "string of sausages". An example of a such a cluster is shown in Fig. 2(left). Unlike the situation for random walk, there can be multiple paths of occupied bonds connecting 0 to $x$. However, for $d>4$ we expect there to be on the order of $n$ pivotal bonds, which are essential for the connection. The pivotal bonds are denoted by bold lines in Fig. 2(left) - the string in the string of sausages. With this picture in mind, we can regard a percolation cluster as a kind of random walk whose vertices are "sausages" and whose steps are the pivotal bonds. There can be no connection from one sausage to a later sausage other than the connection via the pivotal bonds between these sausages, or the pivotal bonds would not be pivotal. This introduces a kind of repulsive interaction between the sausages, but for $d>4$ we expect this interaction to be weak.

Fix $p \in\left[0,\|D\|_{\infty}^{-1}\right]$. As we will argue in Section 3, the generalisation of (2.4) to oriented percolation takes the form

$$
\tau_{n}(x)=p\left(D * \tau_{n-1}\right)(x)+\sum_{m=2}^{n-1} p\left(\pi_{m} * D * \tau_{n-m-1}\right)(x)+\pi_{n}(x) \quad(n \geqslant 1),
$$

where $\pi_{n}(x)$ is defined in Section 3. In particular, $\pi_{n}(x)$ depends on $p$, is invariant under the lattice symmetries, and $\pi_{0}(x)=\pi_{1}(x)=0$. For $n=0$, 1 , we have $\tau_{0}(x)=\delta_{0, x}$ and 

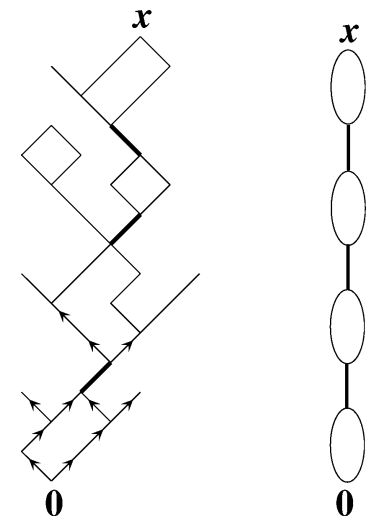

Fig. 2. (Left) A bond configuration. (Right) Schematic depiction of the configuration as a "string of sausages".

$\tau_{1}(x)=p D(x)$, which is consistent with (2.5) (the last two terms on the right side of (2.5) vanish for $n=1,2$ ). The identity (2.5) can be regarded as an inductive definition of the sequence $\pi_{n}(x)$, for $n \geqslant 2$. However, to analyse (2.5) it will be necessary to have a useful representation for $\pi_{n}(x)$, and this is provided in Section 3. Note that (2.4) is of the form (2.5) with $\pi_{n}(x)=0$.

In Section 3, we will express $\pi_{n}(x)$ as

$$
\pi_{n}(x)=\sum_{N=0}^{\infty}(-1)^{N} \pi_{n}^{(N)}(x) .
$$

The terms in the right side of (2.6) are of diminishing importance as $N$ increases, although all make essential contributions. The first term $\pi_{n}^{(0)}(x)$ is zero for $n \leqslant 1$, and is equal to the probability that there is no pivotal bond for the connection from 0 to $x$ for $n \geqslant 2$. Some insight into the expansion (2.5) can be obtained by looking at the contribution to the right side of $(2.5)$ due to $\pi_{n}^{(0)}(x)$. The contribution due to $\pi_{n}^{(0)}(x)$ in the last term of (2.5) arises from configurations in which the string of sausages consists of a single sausage only. The term $\left(p D * \tau_{n-1}\right)(x)$ arises from configurations where there is at least one pivotal bond and the bottom of the first pivotal bond is the origin. This neglects the repulsive interaction mentioned above, since there is no restriction in $\left(p D * \tau_{n-1}\right)(x)$ to guarantee that the first pivotal bond really is pivotal. Similarly, $\sum_{m=2}^{n-1}\left(\pi_{m}^{(0)} * p D * \tau_{n-m-1}\right)(x)$ arises from configurations where there is more than one sausage and the first sausage has "height" at least two (height one is not possible), with the first sausage treated as independent of the cluster above it. The first sausage is in fact not independent of what comes later, due to the repulsive interaction, and therefore corrections are required. The corrections are provided by the terms $N \geqslant 1$ in (2.6), via a sophisticated inclusion-exclusion analysis. The analysis is carried out in detail in Section 3.

There are two formulas for $\pi_{n}(x)$ already available in the literature. Hara and Slade [20] developed an expression for $\pi_{n}(x)$ in terms of sums of nested expectations, by repeated use of inclusion-exclusion. In [20], non-oriented percolation was considered, 
but the expansion of [20] applies more generally, and, in particular, applies to oriented percolation without modification. For oriented percolation, Nguyen and Yang [31] developed an alternate expression for $\pi_{n}(x)$, without nested expectations, by an adaptation of the lace expansion of Brydges and Spencer [8]. The Nguyen-Yang expansion relies on the Markov property of oriented percolation, and does not apply to non-oriented percolation. The functions $\pi_{n}$ that appear in both of these analyses are of course the same, since (2.5) uniquely determines $\pi_{n}$. However, the expansions are different, in the sense that each expansion leads to (2.6) but with different expressions for the $\pi_{n}^{(N)}(x)$. On the other hand, in either expansion, $\pi_{n}^{(N)}(x)$ is nonnegative for all $n, x, N$, and can be represented in terms of Feynman diagrams. The Feynman diagrams are similar in their essential features for the two expansions, and obey similar estimates. We will make use of both expansions, and discuss them in detail in Section 3.

The identity (2.4) can be solved using the Fourier transform to give $\hat{q}_{n}(k)=\left[\hat{q}_{1}(k)\right]^{n}$. Using $q_{1}(x)=p D(x)$, the central limit theorem can then be easily derived. Our method will involve showing that $\pi_{n}(x)$ is small for $p=p_{c}$ if $d>4$ and $n$ and $L$ are both large, so that (2.5) can be regarded as a small perturbation of (2.4), leading to a central limit theorem for the critical two-point function.

\subsubsection{Implementation of the inductive method}

In what follows, we will use the notation $\|f\|_{\infty}=\sup _{x \in \mathbb{Z}^{d}}|f(x)|$ for a function $f: \mathbb{Z}^{d} \rightarrow \mathbb{C}$, and $\|\hat{f}\|_{1}=(2 \pi)^{-d} \int_{[-\pi, \pi]^{d}}|\hat{f}(k)| \mathrm{d}^{d} k$ for a function $\hat{f}:[-\pi, \pi]^{d} \rightarrow \mathbb{C}$.

Our analysis of (2.5) begins by taking its Fourier transform, which gives the recursion relation

$$
\hat{\tau}_{n+1}(k)=p \widehat{D}(k) \hat{\tau}_{n}(k)+p \widehat{D}(k) \sum_{m=2}^{n} \hat{\pi}_{m}(k) \hat{\tau}_{n-m}(k)+\hat{\pi}_{n+1}(k) \quad(n \geqslant 0) .
$$

The right side of (2.7) explicitly involves $\hat{\tau}_{m}(k)$ only for $m \leqslant n$. We will show in Section 4 that it is possible to estimate $\hat{\pi}_{m}(k)$, for all $m \leqslant n+1$, in terms of $\hat{\tau}_{m}(0)$ and $\left\|\tau_{m}\right\|_{\infty}$ with $m \leqslant n$. This opens up the possibility of an inductive analysis of (2.7). A general approach to this type of inductive analysis is given in [27], and we will apply a general theorem of [27] to (2.7) to prove Theorem 1.1.

To put (2.7) into the notation of [27], we introduce the following notation. (In [27], $p$ is written as $z$.) Let

$$
\begin{aligned}
& f_{n}(k ; p)=\hat{\tau}_{n}(k), \quad e_{n}(k ; p)=\hat{\pi}_{n}(k) \quad(n \geqslant 0), \\
& g_{1}(k ; p)=p \widehat{D}(k), \quad g_{n}(k ; p)=p \widehat{D}(k) \hat{\pi}_{n-1}(k) \quad(n \geqslant 2),
\end{aligned}
$$

where the dependence of $\tau$ and $\pi$ on $p$ has been made explicit in $e, f, g$. Note that $\hat{\tau}_{1}(k)=p \widehat{D}(k)$. The recursion relation (2.7) can then be written as

$$
f_{0}(k ; p)=1, \quad f_{n+1}(k ; p)=\sum_{m=1}^{n+1} g_{m}(k ; p) f_{n+1-m}(k ; p)+e_{n+1}(k ; p) \quad(n \geqslant 0) .
$$

Since $\pi_{m}=0$ for $m \leqslant 1$, we have $e_{1}(k ; p)=g_{2}(k ; p)=0$. 
The result of Theorem 1.1 was shown in [27] to hold for solutions of the recursion relation (2.10), subject to a certain set of assumptions on $\hat{e}_{n}$ and $\hat{g}_{n}$. Moreover, subject to these assumptions, it is shown in [27] that the critical point is given implicitly by the equation

$$
p_{c}=\frac{1}{1+\sum_{m=2}^{\infty} \hat{\pi}_{m}\left(0 ; p_{c}\right)},
$$

and that the constants $A, v$ of Theorem 1.1 are given by

$$
\begin{aligned}
& A=\left[p_{c}+p_{c}^{2} \sum_{m=2}^{\infty} m \hat{\pi}_{m}\left(0 ; p_{c}\right)\right]^{-1}, \\
& v=p_{c} A\left[1-p_{c} \sigma^{-2} \sum_{m=2}^{\infty} \nabla^{2} \hat{\pi}_{m}\left(0 ; p_{c}\right)\right],
\end{aligned}
$$

where we have added an argument $p_{c}$ to emphasise that $p$ is critical for the evaluation of $\pi_{m}$ on the right sides of (2.11)-(2.13). Convergence of the series in (2.11)-(2.13), for $d>4$, will follow from Proposition 2.2 below.

As described in [27, Section 1.4.2], the only substantial assumptions to verify are Assumptions E and G of [27], which we restate here together as Assumption 2.1. Its statement involves the small parameter

$$
\beta=L^{-d}
$$

and $\sigma^{2}$ of (1.3). An essential aspect of the assumption is that bounds on $f_{m}$ for $1 \leqslant m \leqslant n$ imply bounds on $e_{m}$ and $g_{m}$ for all $2 \leqslant m \leqslant n+1$. It is the inclusion of $m=n+1$ for the implied bounds that allows the inductive analysis of [27] to proceed.

Assumption 2.1. - There is an $L_{0}$, an interval $I \subset[1-\alpha, 1+\alpha]$ with $\alpha \in(0,1)$, and a function $K_{f} \mapsto C_{g}\left(K_{f}\right)$, such that if the bounds

$$
\left|f_{m}(0 ; p)\right| \leqslant K_{f}, \quad\left|\nabla^{2} f_{m}(0 ; p)\right| \leqslant K_{f} \sigma^{2} m, \quad\left\|\widehat{D}^{2} f_{m}(\cdot ; p)\right\|_{1} \leqslant K_{f} \beta m^{-d / 2}
$$

hold for some $K_{f}>1, L \geqslant L_{0}, p \in I$ and for all $1 \leqslant m \leqslant n$, then for that $L$ and $p$, and for all $k \in[-\pi, \pi]^{d}$ and $2 \leqslant m \leqslant n+1$, the following bounds hold:

$$
\begin{gathered}
\left|e_{m}(k ; p)\right| \leqslant C_{g}\left(K_{f}\right) \beta m^{-d / 2}, \quad\left|g_{m}(k ; p)\right| \leqslant C_{g}\left(K_{f}\right) \beta m^{-d / 2}, \\
\left|\partial_{p} g_{m}(0 ; p)\right| \leqslant C_{g}\left(K_{f}\right) \beta m^{-(d-2) / 2}, \\
\left|\nabla^{2} g_{m}(0 ; p)\right| \leqslant C_{g}\left(K_{f}\right) \sigma^{2} \beta m^{-(d-2) / 2}, \\
\left|e_{m}(k ; p)-e_{m}(0 ; p)\right| \leqslant C_{g}\left(K_{f}\right)[1-\widehat{D}(k)] \beta m^{-(d-2) / 2}, \\
\left|g_{m}(k ; p)-g_{m}(0 ; p)-[1-\widehat{D}(k)] \sigma^{-2} \nabla^{2} g_{m}(0 ; p)\right| \\
\leqslant C_{g}\left(K_{f}\right) \beta[1-\widehat{D}(k)]^{1+\varepsilon^{\prime}} m^{-\left(d-2-2 \varepsilon^{\prime}\right) / 2},
\end{gathered}
$$

with (2.20) valid for any $\varepsilon^{\prime} \in[0, \varepsilon \wedge 1]$. 
The validity of (2.20) for $\varepsilon^{\prime} \in[0, \varepsilon \wedge 1]$ differs from the requirement $\varepsilon^{\prime} \in[0, \varepsilon]$ in the statement of Assumption $\mathrm{G}$ in [27]. However, we may assume that $\varepsilon \leqslant 1$ without loss of generality, since the statements of Theorems 1.1-1.2 involve only $\varepsilon \wedge 1$, and since (1.2) implies the same estimate for $\varepsilon^{\prime} \leqslant \varepsilon$, by Hölder's inequality.

Note that Assumption 2.1 does not assume that (2.15) holds, but rather that if (2.15) holds then (2.16)-(2.20) must hold. Once we establish Assumption 2.1, Theorem 1.1 then follows immediately from [27, Theorem 1.1]. Moreover, as explained in [27, Section 1.3], it is a consequence of establishing Assumption 2.1 that, for $p=p_{c}$, (2.15) holds for all $m \geqslant 1$ and (2.16)-(2.20) hold for all $m \geqslant 2$. Assumption 2.1 will follow from the following proposition.

Proposition 2.2. - Assume (2.15), for $p \in I$ and $1 \leqslant m \leqslant n$. Then there is $a \beta_{0}>0$ and a finite $C$ (both depending on $K_{f}$ but not on $\varepsilon^{\prime}$ ), such that for $\beta \leqslant \beta_{0}, \varepsilon^{\prime} \in[0, \varepsilon \wedge 1]$, and for all $2 \leqslant m \leqslant n+1$,

(i)

$$
\sum_{x}|x|^{q}\left|\pi_{m}(x)\right| \leqslant C \beta \sigma^{q} m^{-(d-q) / 2} \quad(q=0,2,4)
$$

(ii)

$$
\begin{aligned}
& \left|\hat{\pi}_{m}(k)-\hat{\pi}_{m}(0)-[1-\widehat{D}(k)] \sigma^{-2} \nabla^{2} \hat{\pi}_{m}(0)\right| \\
& \leqslant C \beta[1-\widehat{D}(k)]^{1+\varepsilon^{\prime}} m^{-\left(d-2-2 \varepsilon^{\prime}\right) / 2},
\end{aligned}
$$

(iii)

$$
\left|p \partial_{p} \hat{\pi}_{m}(0)\right| \leqslant C \beta m^{-(d-2) / 2} .
$$

The proof of Proposition 2.2 is deferred to Section 4. We now show that it implies Assumption 2.1. As discussed above, it therefore gives the proof of Theorem 1.1 and establishes the estimates (2.15).

Verification of Assumption 2.1 assuming Proposition 2.2. - By definition, we have that $e_{m}(k ; p)=\hat{\pi}_{m}(k), g_{2}(k ; p)=0$, and $g_{m}(k ; p)=p \widehat{D}(k) \hat{\pi}_{m-1}(k)$ for all $m \geqslant 3$. The bounds (2.16) therefore follow immediately from (2.21) with $q=0$, for $2 \leqslant m \leqslant n+1$. By definition,

$$
\partial_{p} g_{m}(0 ; p)=\hat{\pi}_{m-1}(0)+p \partial_{p} \hat{\pi}_{m-1}(0) .
$$

The bound (2.17) therefore follows from (2.21) with $q=0$ and (2.23). By symmetry,

$$
\nabla^{2} g_{m}(0 ; p)=-p \sigma^{2} \hat{\pi}_{m-1}(0)+p \nabla^{2} \hat{\pi}_{m-1}(0) .
$$

The bound (2.18) therefore follows from (2.21) with $q=0,2$.

For (2.19), we use (2.22) with $\varepsilon^{\prime}=0$ to obtain

$$
\begin{aligned}
\left|e_{m}(0 ; p)-e_{m}(k ; p)\right| & =\left|\hat{\pi}_{m}(k)-\hat{\pi}_{m}(0)\right| \\
& \leqslant[1-\widehat{D}(k)]\left[\sigma^{-2}\left|\nabla^{2} \hat{\pi}_{m}(0)\right|+C \beta m^{-(d-2) / 2}\right],
\end{aligned}
$$

and apply (2.21) with $q=2$.

For (2.20), by definition and symmetry we have 


$$
\begin{aligned}
& g_{m}(k ; p)-g_{m}(0 ; p)-[1-\widehat{D}(k)] \sigma^{-2} \nabla^{2} g_{m}(0 ; p) \\
& =p\left(\hat{\pi}_{m-1}(k)-\hat{\pi}_{m-1}(0)-[1-\widehat{D}(k)] \sigma^{-2} \nabla^{2} \hat{\pi}_{m-1}(0)\right) \\
& \quad+p[1-\widehat{D}(k)]\left[\hat{\pi}_{m-1}(0)-\hat{\pi}_{m-1}(k)\right] .
\end{aligned}
$$

The second term on the right side is better than required, by (2.19). The first term gives the required bound, by (2.22).

\subsection{The $r$-point functions, $r \geqslant 3$}

Now we move on to the $r$-point functions with $r \geqslant 3$, and give an introduction to our expansion methods. Together with the inductive analysis of the two-point function, these expansion methods constitute the part of this paper that is essentially new. Full details of the expansion are deferred to Sections 5 and 6. For the remainder of the paper, it will be convenient to use new notation for sites in $\mathbb{Z}^{d} \times \mathbb{N}$. We write $\Lambda=\mathbb{Z}^{d} \times \mathbb{N}$, and we write a typical element of $\Lambda$ as $\boldsymbol{x}$ rather than $(x, n)$ as was used until now. We fix $p=p_{c}$ throughout Section 2.2 for simplicity, though the discussion also applies without change when $p<p_{c}$. We begin with an overview of the expansion.

\subsubsection{Overview of the expansion}

The basic picture underlying the expansion for the two-point function is that a cluster connecting $\mathbf{0}$ to $\boldsymbol{x}$ can be viewed as a string of sausages. For connections from the origin to multiple points $\overrightarrow{\boldsymbol{x}}=\left(\boldsymbol{x}_{1}, \ldots, \boldsymbol{x}_{r-1}\right)$, the corresponding picture is a "tree of sausages" as depicted in Fig. 3. In the tree of sausages, the "string" represents the union over $i=1, \ldots, r-1$ of the occupied pivotal bonds for the connections $\mathbf{0} \rightarrow \boldsymbol{x}_{i}$. We regard this picture as corresponding to a kind of branching random walk, with the sites of the walk being the sausages and the steps of the walk being effectively independent when $d>4$. We will use this picture now to give an overview of the expansions we will derive in Sections 5 and 6.

The basic idea is that we may regard the configuration depicted in Fig. 3 as approximately a product of four independent factors. These factors are the following:

(1) a two-point function corresponding to the connection from the origin to the bottom of the pivotal bond leading into the first branching sausage, i.e., the sausage from which the branches to $\boldsymbol{x}_{1}, \boldsymbol{x}_{2}$ and to $\boldsymbol{x}_{3}$ emerge;

(2) the first branching sausage together with the pivotal bond leading into it and the two pivotal bonds leading out of it;

(3) a two-point function corresponding to the branch to $\boldsymbol{x}_{3}$;

(4) a three-point function corresponding to the branch to $\boldsymbol{x}_{1}, \boldsymbol{x}_{2}$.

The above decomposition into a product is only approximate, and corrections are taken into account in an iterative fashion leading to an expansion. The net effect of the first branching sausage (item (2) above), following the expansion, is to produce a certain factor $\psi$ that is analogous to $\pi$. However, whereas $\pi$ is a kind of two-point function, $\psi$ will be a kind of three-point function. Our estimates will show that the first branching sausage is typically small and scales to a point in the scaling limit. Its net contribution is to provide the vertex factor $V$ of Theorem 1.2. The three-point function of item (4) will be treated recursively, and is approximately given by a convolution of 


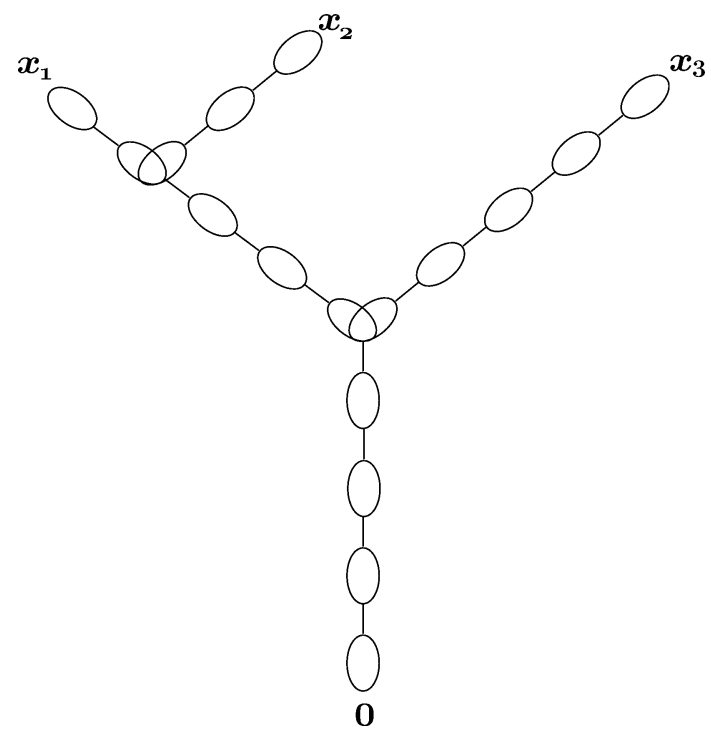

Fig. 3. Schematic depiction of a configuration as a "tree of sausages".

three two-point functions with another factor of $\psi$. This leads to a decomposition of the configuration of Fig. 3 into a product of five two-point functions, and two factors $\psi$ which each reduce to a factor $V$ in the scaling limit. This produces the contribution to the asymptotic behaviour (1.21) of the four-point function due to the shape depicted in Fig. 3. The factor $n^{2}$ in (1.21) arises from a time rescaling of the branching locations.

To describe the expansion in more detail, we use the following notation. For $r \geqslant 3$, let

$$
J=\{1,2, \ldots, r-1\}, \quad J_{1}=J \backslash\{1\} .
$$

For $I=\left\{i_{1}, \ldots, i_{s}\right\} \subset J$, we write $\overrightarrow{\boldsymbol{x}}_{I}=\left\{\boldsymbol{x}_{i_{1}}, \ldots, \boldsymbol{x}_{i_{s}}\right\}$ and $\overrightarrow{\boldsymbol{x}}_{I}-\boldsymbol{y}=\left\{\boldsymbol{x}_{i_{1}}-\boldsymbol{y}, \ldots\right.$, $\left.\boldsymbol{x}_{i_{s}}-\boldsymbol{y}\right\}$. Given a subset $I \subset J_{1}$, let $r_{1}=|J \backslash I|+1$ and $r_{2}=|I|+1$. We will use the notation $D(v)=D((v, j))=D(v) \delta_{j, 1}$ of (2.2).

We focus on the first pivotal bond for the connection from $\boldsymbol{0}$ to $\boldsymbol{x}_{1}$, thereby assigning a special status to $\boldsymbol{x}_{1}$. If there is no pivotal bond for $\mathbf{0} \rightarrow \boldsymbol{x}_{1}$, the configuration contributes to an error term and we will not consider this case in detail now. To a first approximation, we regard the first sausage as being independent of the remaining sausage, allowing for factorisation of expectation. The first sausage may contain none of the components of $\overrightarrow{\boldsymbol{x}}_{J_{1}}$, as in Fig. 3, or it may contain any nonempty subset of $\overrightarrow{\boldsymbol{x}}_{J_{1}}$. Taking into account corrections to the approximation, in Section 5 we will prove an identity

$$
\tau^{(r)}\left(\overrightarrow{\boldsymbol{x}}_{J}\right)=A^{(r)}\left(\overrightarrow{\boldsymbol{x}}_{J}\right)+\sum_{I \subset J_{1}} \sum_{\boldsymbol{v}_{1}} B^{\left(r_{2}+1\right)}\left(\boldsymbol{v}_{1}, \overrightarrow{\boldsymbol{x}}_{I}\right) \tau^{\left(r_{1}\right)}\left(\overrightarrow{\boldsymbol{x}}_{J \backslash I}-\boldsymbol{v}_{1}\right) .
$$

Here, the set $I$ indicates which $\overrightarrow{\boldsymbol{x}}_{I}$ are in the first sausage, and the factor $\tau^{\left(r_{1}\right)}\left(\overrightarrow{\boldsymbol{x}}_{J \backslash I}-\boldsymbol{v}_{1}\right)$ gives the desired item (4) in the list above, in the case when $I$ is nonempty. The derivation of (2.29) is a nontrivial procedure, and both $A^{(r)}\left(\overrightarrow{\boldsymbol{x}}_{J}\right)$ and $B^{\left(r_{2}+1\right)}\left(\boldsymbol{v}_{1}, \overrightarrow{\boldsymbol{x}}_{I}\right)$ represent many-point generalisations of the function $\pi$ arising in the expansion for the 
two-point function. In particular, $A^{(r)}(\overrightarrow{\boldsymbol{x}})$ includes, among other terms, the probability that there is no pivotal bond for the connection $\mathbf{0} \rightarrow \boldsymbol{x}_{1}$. To leading order, $B^{\left(r_{2}+1\right)}\left(\boldsymbol{v}_{1}, \overrightarrow{\boldsymbol{x}}_{I}\right)$ represents the first sausage for the connection $\mathbf{0} \rightarrow \boldsymbol{x}_{1}$, together with the first pivotal bond $\left(\boldsymbol{u}_{1}, \boldsymbol{v}_{1}\right)$ for the connection, and a branch leading to $\overrightarrow{\boldsymbol{x}}_{I}$.

When $I$ is not empty, we perform a second expansion. For the leading contribution to $B^{\left(r_{2}+1\right)}\left(\boldsymbol{v}_{1}, \overrightarrow{\boldsymbol{x}}_{I}\right)$, the second expansion allows for a decoupling of the branch to $\overrightarrow{\boldsymbol{x}}_{I}$. The second expansion leads to a result of the form

$$
B^{\left(r_{2}+1\right)}\left(\boldsymbol{v}_{1}, \overrightarrow{\boldsymbol{x}}_{I}\right)=\sum_{\boldsymbol{v}_{2}} C\left(\boldsymbol{v}_{1}, \boldsymbol{v}_{2}\right) \tau^{\left(r_{2}\right)}\left(\overrightarrow{\boldsymbol{x}}_{I}-\boldsymbol{v}_{2}\right)+R^{\left(r_{2}+1\right)}\left(\boldsymbol{v}_{1}, \overrightarrow{\boldsymbol{x}}_{I}\right),
$$

where $R^{\left(r_{2}+1\right)}\left(\boldsymbol{v}_{1}, \overrightarrow{\boldsymbol{x}}_{I}\right)$ is an error term. To a first approximation, $C\left(\boldsymbol{v}_{1}, \boldsymbol{v}_{2}\right)$ represents a truncated branching sausage at $\mathbf{0}$ together with the pivotal bonds ending at $\boldsymbol{v}_{1}$ and $\boldsymbol{v}_{2}$, with two branches removed. In particular, $C\left(\boldsymbol{v}_{1}, \boldsymbol{v}_{2}\right)$ is independent of $I$. The leading contribution to $C\left(\boldsymbol{v}_{1}, \boldsymbol{v}_{2}\right)$ is $p^{2} D\left(\boldsymbol{v}_{1}\right) D\left(\boldsymbol{v}_{2}\right)$, corresponding to the case where the truncated branching sausage at $\mathbf{0}$ is the single vertex $\mathbf{0}$. For details, see Section 6 , where (2.30) is derived. The term $C\left(\boldsymbol{v}_{1}, \boldsymbol{v}_{2}\right)$ represents most of item (2) in the above list, but it lacks the lower pivotal bond. This will be corrected in Section 2.2.2, where we will return to items (1)-(4) above.

For $r=2$, only the term $I=\emptyset$ exists and (2.29) becomes

$$
\tau^{(2)}(\boldsymbol{x})=A^{(2)}(\boldsymbol{x})+\sum_{\boldsymbol{v}_{1} \in \Lambda} B^{(2)}\left(\boldsymbol{v}_{1}\right) \tau^{(2)}\left(\boldsymbol{x}-\boldsymbol{v}_{1}\right)
$$

Comparing with (2.5), we see that

$$
A^{(2)}(\boldsymbol{x})=\delta_{\mathbf{0}, \boldsymbol{x}}+\pi(\boldsymbol{x}), \quad B^{(2)}\left(\boldsymbol{v}_{1}\right)=\sum_{\boldsymbol{u} \in \Lambda} A^{(2)}(\boldsymbol{u}) p D\left(\boldsymbol{v}_{1}-\boldsymbol{u}\right) .
$$

\subsubsection{The main identity and estimates}

To simplify the notation, we write $\overrightarrow{\boldsymbol{x}}$ in place of $\overrightarrow{\boldsymbol{x}}_{J}=\left(\boldsymbol{x}_{1}, \ldots, \boldsymbol{x}_{r-1}\right)$. To isolate the one term on the right side of (2.29) in which $\tau^{(r)}$ occurs, we define

$$
\alpha^{(r)}(\overrightarrow{\boldsymbol{x}})=A^{(r)}(\overrightarrow{\boldsymbol{x}})+\sum_{I \subset J_{1}: I \neq \emptyset} \sum_{\boldsymbol{v}_{1}} B^{\left(r_{2}+1\right)}\left(\boldsymbol{v}_{1}, \overrightarrow{\boldsymbol{x}}_{I}\right) \tau^{\left(r_{1}\right)}\left(\overrightarrow{\boldsymbol{x}}_{J \backslash I}-\boldsymbol{v}_{1}\right),
$$

so that

$$
\tau^{(r)}(\overrightarrow{\boldsymbol{x}})=\alpha^{(r)}(\overrightarrow{\boldsymbol{x}})+\sum_{\boldsymbol{v} \in \Lambda} B^{(2)}(\boldsymbol{v}) \tau^{(r)}(\overrightarrow{\boldsymbol{x}}-\boldsymbol{v}) .
$$

In particular, comparing with (2.31), $\alpha^{(2)}$ is equal to $A^{(2)}$ of (2.32).

The recursion (2.34) can be solved by iteration. For this, we let

$$
(f * g)(\boldsymbol{x})=\sum_{\boldsymbol{v} \in \Lambda} f(\boldsymbol{v}) g(\boldsymbol{x}-\boldsymbol{v})
$$


denote the space-time convolution of $f$ and $g$, and we define

$$
v(\boldsymbol{x})=\sum_{l=0}^{\infty}\left(B^{(2)}\right)^{* l}(\boldsymbol{x}) .
$$

Here, $\left(B^{(2)}\right)^{* l}$ denotes the $l$-fold space-time convolution of $B^{(2)}$ with itself, with $\left(B^{(2)}\right)^{* 0}(\boldsymbol{x})=\delta_{\boldsymbol{0}, \boldsymbol{x}}$. The sum over $\boldsymbol{l}$ in (2.36) terminates after finitely many terms, since by definition $B^{(2)}((x, n)) \neq 0$ only if $n>0$. Then (2.34) can be iterated to give

$$
\tau^{(r)}(\overrightarrow{\boldsymbol{x}})=\sum_{\boldsymbol{v} \in \Lambda} \nu(\boldsymbol{v}) \alpha^{(r)}(\overrightarrow{\boldsymbol{x}}-\boldsymbol{v})
$$

The function $v$ can be identified as follows. Extracting the $l=0$ term from (2.36), using (2.32) to write one factor of $B^{(2)}$ as $p D * A^{(2)}$ for the terms with $l \geqslant 1$, and using (2.37) with $r=2$ (in which case $\alpha^{(2)}=A^{(2)}$ ), it follows that

$$
v(\boldsymbol{x})=\delta_{\mathbf{0}, \boldsymbol{x}}+p\left(D * A^{(2)} * v\right)(\boldsymbol{x})=\delta_{\mathbf{0}, \boldsymbol{x}}+p\left(D * \tau^{(2)}\right)(\boldsymbol{x}) .
$$

Subsituting (2.38) into (2.37), the solution to (2.34) is then given by

$$
\tau^{(r)}(\overrightarrow{\boldsymbol{x}})=\alpha^{(r)}(\overrightarrow{\boldsymbol{x}})+p \sum_{\boldsymbol{v} \in \Lambda}\left(\tau^{(2)} * D\right)(\boldsymbol{v}) \alpha^{(r)}(\overrightarrow{\boldsymbol{x}}-\boldsymbol{v}),
$$

which, using (2.32) and $\alpha^{(2)}=A^{(2)}$, recovers (2.31) when $r=2$.

Our next step is to write $\alpha^{(r)}=f^{(r)}+g^{(r)}$, where $f^{(r)}$ is the contribution that will provide the leading behaviour of the right side of (2.39), while $g^{(r)}$ gives an error term. This is achieved by substituting (2.30) into (2.33) and setting

$$
\begin{aligned}
f^{(r)}(\overrightarrow{\boldsymbol{x}}) & =\sum_{I \subset J_{1}: I \neq \emptyset} \sum_{\boldsymbol{v}_{1}, \boldsymbol{v}_{2}} C\left(\boldsymbol{v}_{1}, \boldsymbol{v}_{2}\right) \tau^{\left(r_{1}\right)}\left(\overrightarrow{\boldsymbol{x}}_{J \backslash I}-\boldsymbol{v}_{1}\right) \tau^{\left(r_{2}\right)}\left(\overrightarrow{\boldsymbol{x}}_{I}-\boldsymbol{v}_{2}\right), \\
g^{(r)}(\overrightarrow{\boldsymbol{x}}) & =A^{(r)}(\vec{x})+\sum_{I \subset J_{1}: I \neq \emptyset} \sum_{\boldsymbol{v}_{1}} R^{\left(r_{2}+1\right)}\left(\boldsymbol{v}_{1}, \overrightarrow{\boldsymbol{x}}_{I}\right) \tau^{\left(r_{1}\right)}\left(\overrightarrow{\boldsymbol{x}}_{J \backslash I}-\boldsymbol{v}_{1}\right) .
\end{aligned}
$$

Defining

$$
\begin{aligned}
\psi\left(\boldsymbol{y}_{1}, \boldsymbol{y}_{2}\right) & =\sum_{\boldsymbol{u} \in \Lambda} p D(\boldsymbol{u}) C\left(\boldsymbol{y}_{1}-\boldsymbol{u}, \boldsymbol{y}_{2}-\boldsymbol{u}\right), \\
\varphi^{(r)}(\overrightarrow{\boldsymbol{x}}) & =\alpha^{(r)}(\overrightarrow{\boldsymbol{x}})+\sum_{\boldsymbol{v} \in \Lambda} p\left(\tau^{(2)} * D\right)(\boldsymbol{v}) g^{(r)}(\overrightarrow{\boldsymbol{x}}-\boldsymbol{v}),
\end{aligned}
$$

(2.39) becomes

$$
\begin{aligned}
\tau^{(r)}(\overrightarrow{\boldsymbol{x}})= & \sum_{\boldsymbol{v}, \boldsymbol{v}_{1}, \boldsymbol{v}_{2} \in \Lambda} \tau^{(2)}(\boldsymbol{v}) \psi\left(\boldsymbol{v}_{1}-\boldsymbol{v}, \boldsymbol{v}_{2}-\boldsymbol{v}\right) \\
& \times \sum_{I \subset J_{1}:|I| \geqslant 1} \tau^{\left(r_{1}\right)}\left(\overrightarrow{\boldsymbol{x}}_{J \backslash I}-\boldsymbol{v}_{1}\right) \tau^{\left(r_{2}\right)}\left(\overrightarrow{\boldsymbol{x}}_{I}-\boldsymbol{v}_{2}\right)+\varphi^{(r)}(\overrightarrow{\boldsymbol{x}}),
\end{aligned}
$$

where we recall that $r_{1}=|J \backslash I|+1$ and $r_{2}=|I|+1$. The first term on the right side of (2.44) is the main term and is depicted schematically in Fig. 4. In Fig. 4 and in the main 


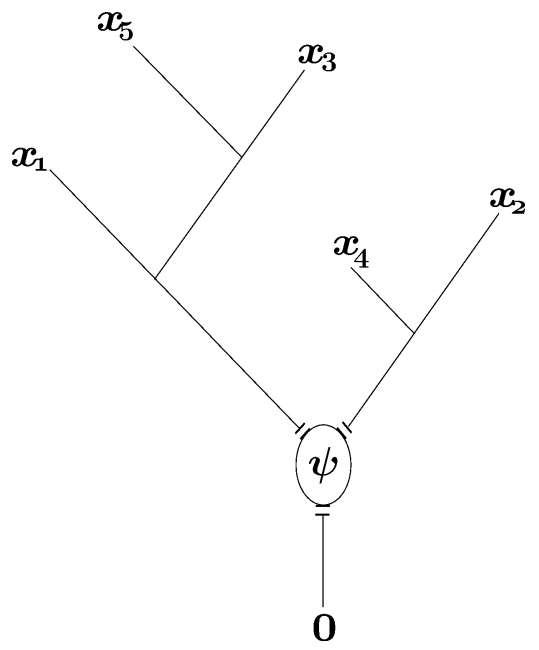

Fig. 4. Schematic depiction of the first term on the right side of (2.44).

term of (2.44), each of items (1)-(4) from Section 2.2.1 is clearly visible. The leading contribution to $\psi\left(\boldsymbol{y}_{1}, \boldsymbol{y}_{2}\right)$ is

$$
\psi_{2,2}\left(y_{1}, y_{2}\right)=\sum_{u} p^{3} D(u) D\left(y_{1}-u\right) D\left(y_{2}-u\right)
$$

using the leading contribution to $C$ described under (2.30). Here, we are writing $\psi_{m_{1}, m_{2}}\left(y_{1}, y_{2}\right)$ for $\psi\left(\left(y_{1}, m_{1}\right),\left(y_{2}, m_{2}\right)\right)$. By definition, $\psi_{m_{1}, m_{2}}\left(y_{1}, y_{2}\right)=0$ if one of $m_{1}$ or $m_{2}$ is less than 2 , due to the inclusion of the pivotal bonds to $\boldsymbol{v}_{1}$ and $\boldsymbol{v}_{2}$ in $C\left(\boldsymbol{v}_{1}, \boldsymbol{v}_{2}\right)$.

We will analyse (2.44) using the Fourier transform. We write $\vec{n}=\left(n_{1}, \ldots, n_{r-1}\right)$ and $\vec{k}=\left(k_{1}, \ldots, k_{r-1}\right)$. For $I \subset\{1,2, \ldots, r-1\}$, we write $\vec{k}_{I}=\left(k_{i}\right)_{i \in I}, k_{I}=\sum_{i \in I} k_{i}$ and $k=\sum_{i=1}^{r-1} k_{i}$. We also write $\underline{n}_{I}=\min _{i \in I} n_{i}, \underline{n}=\min _{i} n_{i}$ and define $\vec{n}_{I}-m$ to be the vector obtained by subtracting $m$ from each component of $\vec{n}_{I}$. With this notation, the Fourier transform of (2.44) becomes

$$
\begin{aligned}
\hat{\tau}_{\vec{n}}^{(r)}(\vec{k})= & \sum_{n_{0}=0}^{\underline{n}} \hat{\tau}_{n_{0}}^{(2)}(k) \sum_{I \subset J_{1}:|I| \geqslant 1} \sum_{m_{1}=2}^{\underline{n}_{J \backslash I}-n_{0}} \sum_{m_{2}=2}^{\underline{n}_{I}-n_{0}} \hat{\psi}_{m_{1}, m_{2}}\left(k_{J \backslash I}, k_{I}\right) \\
& \times \hat{\tau}_{\vec{n}_{J \backslash I}^{\left(r_{1}\right)}-m_{1}-n_{0}}\left(\vec{k}_{J \backslash I}\right) \hat{\tau}_{\vec{n}_{I}-m_{2}-n_{0}}^{\left(r_{2}\right)}\left(\vec{k}_{I}\right)+\hat{\varphi}_{\vec{n}}^{(r)}(\vec{k}) .
\end{aligned}
$$

The identity (2.46) is our main identity and will be our point of departure for analysing the $r$-point functions for $r \geqslant 3$. Apart from $\psi$ and $\varphi^{(r)}$, the right side of (2.44) involves the $s$-point functions with $s=2, r_{1}, r_{2}$. Since $r_{1}+r_{2}=r+1$ and $r_{1}, r_{2} \geqslant 2$, it follows that $r_{1}$ and $r_{2}$ are both strictly less than $r$. This allows for an analysis by induction on $r$, with the $r=2$ case given by the result of Theorem 1.1. The term involving $\psi$ is the main term, whereas $\varphi^{(r)}$ will turn out to be an error term.

The analysis will be based on the following important proposition, whose proof is deferred to Section 7. The proof of Proposition 2.3 will involve showing that $\psi$ and $\varphi^{(r)}$ can be estimated in terms of $\hat{\tau}_{m}(0)$ and $\left\|\tau_{m}\right\|_{\infty}$, which have been controlled already in Theorem 1.1. The decay in $m_{1}, m_{2}$ for $\hat{\psi}_{m_{1}, m_{2}}\left(k_{1}, k_{2}\right)$ given in the proposition is a 
more precise statement of our previous comment that the branching sausage (item (2) of Section 2.2.1) is typically small. In the statement of the proposition, $\nabla_{i}$ represents partial differentiation with respect to $k_{i}$.

Proposition 2.3. - Fix $d>4, \delta \in\left(0,1 \wedge \varepsilon \wedge \frac{d-4}{2}\right)$ and $p=p_{c}$. Let $\bar{n}$ denote the second-largest element of $\left\{n_{1}, \ldots, n_{r-1}\right\}$. There exist constants $C_{\psi}, C_{\varphi}^{(r)}>0$ (independent of $L)$ and $L_{0}(d)$, such that for all $L \geqslant L_{0}, q \in\{0,2\}, m_{i} \geqslant 2, \vec{n}, r \geqslant 3$ and $k_{i} \in[-\pi, \pi]^{d}$, the following bounds hold:

(i) $\nabla_{i} \hat{\psi}_{m_{1}, m_{2}}(0,0)=0$ and

$$
\left|\nabla_{i}^{q} \hat{\psi}_{m_{1}, m_{2}}\left(k_{1}, k_{2}\right)\right| \leqslant C_{\psi} \sigma^{q} m_{i}^{q / 2}\left(m_{1} \vee m_{2}\right)^{-d / 2},
$$

(ii)

$$
\left|\hat{\varphi}_{\vec{n}}^{(r)}(\vec{k})\right| \leqslant C_{\varphi}^{(r)} \bar{n}^{r-2-\delta} .
$$

Moreover, for $\left(m_{1}, m_{2}\right) \neq(2,2)$, a factor $\beta$ may be included in the right side of (2.47).

It follows from Proposition 2.3(i) that the constant $V$ defined by

$$
V=\sum_{m_{1}, m_{2}=2}^{\infty} \hat{\psi}_{m_{1}, m_{2}}(0,0)
$$

with $p=p_{c}$, is finite. This is the constant $V$ of Theorem 1.2. Since $\hat{\psi}_{2,2}(0,0)=p_{c}^{3}=$ $1+\mathcal{O}(\beta)$ by (2.45) and (1.12), it follows from the final remark in the statement of Proposition 2.3 that

$$
V=1+\mathcal{O}(\beta) .
$$

This establishes the claim on $V$ of Theorem 1.2.

\subsection{Induction on $r$}

In this section, we prove Theorem 1.2 assuming (2.46) and Proposition 2.3. We fix $p=p_{c}$ throughout this section. The formulas (1.25) for the characteristic functions of the moment measures of the canonical measure of super-Brownian motion can be written as

$$
\widehat{M}_{\vec{t}}^{(r-1)}(\vec{k})= \begin{cases}\mathrm{e}^{-|k|^{2} t / 2 d} & (r=2), \\ \sum_{\alpha \in \Sigma_{r}} \int_{R_{\bar{t}}(\alpha)} \mathrm{d} \bar{s} \prod_{\ell=1}^{2 r-3} \mathrm{e}^{-\left|k_{\ell}(\alpha)\right|^{2 \bar{s}_{\ell} / 2 d}} & (r \geqslant 3) .\end{cases}
$$

Let $\bar{n}$ denote the second-largest element of $\left\{n_{1}, \ldots, n_{r-1}\right\}$. We now prove that for $d>4$ there are positive constants $L_{0}=L_{0}(d)$ and $V=V(d, L)$ such that for $p=p_{c}, L \geqslant L_{0}$ and $\delta \in\left(0,1 \wedge \varepsilon \wedge \frac{d-4}{2}\right)$, we have

$$
\hat{\tau}_{\vec{n}}^{(r)}\left(\vec{k} / \sqrt{v \sigma^{2} n}\right)=A\left(A^{2} V n\right)^{r-2}\left[\widehat{M}_{\vec{n} / n}^{(r-1)}(\vec{k})+\mathcal{O}\left((\bar{n}+1)^{-\delta}\right)\right] \quad(r \geqslant 3)
$$

uniformly in $n \geqslant \bar{n}$ and in $\vec{k} \in \mathbb{R}^{d(r-1)}$ with $\sum_{i=1}^{r-1}\left|k_{i}\right|^{2}$ bounded. Since the $\widehat{M}_{\vec{t}}^{(r-1)}(\vec{k})$ are smooth functions of $\vec{t}$, proving the above is sufficient to prove Theorem 1.2. 
We will prove (2.52) by induction on $r$, with the case $r=2$ given by Theorem 1.1 . Indeed, Theorem 1.1(a) gives

$$
\begin{aligned}
\hat{\tau}_{n_{1}}\left(k / \sqrt{v \sigma^{2} n}\right) & =\hat{\tau}_{n_{1}}\left(k n_{1}^{1 / 2} n^{-1 / 2} / \sqrt{v \sigma^{2} n_{1}}\right) \\
& =A\left[\mathrm{e}^{-|k|^{2} n_{1} / 2 d n}+\mathcal{O}\left(\left(n_{1}+1\right)^{-\delta}\right)\right],
\end{aligned}
$$

using the facts that $|k|^{2}$ is bounded, $n_{1} \leqslant n$, and $\delta<\frac{d-4}{2}$.

Before proceeding with the proof of Theorem 1.2, we first recall the following standard recursion relation for the moment measures $\widehat{M}_{\vec{t}}^{(l)}(\vec{k})$ :

$$
\widehat{M}_{\vec{t}}^{(r-1)}(\vec{k})=\int_{0}^{\frac{t}{}} \mathrm{~d} t \widehat{M}_{t}^{(1)}(k) \sum_{I \subset J_{1}:|I| \geqslant 1} \widehat{M}_{\vec{t}_{J \backslash I}-t}^{\left(r_{1}-1\right)}\left(\vec{k}_{J \backslash I}\right) \widehat{M}_{\vec{t}_{I}-t}^{\left(r_{2}-1\right)}\left(\vec{k}_{I}\right) \quad(r \geqslant 3),
$$

where $\underline{t}=\min _{i} t_{i}, k=\sum_{i=1}^{r-1} k_{j}$, and $r_{1}=|J \backslash I|+1, r_{2}=|I|+1$. This recursion can be understood from the fact that a shape $\alpha \in \Sigma_{r}$ contributing to the left side of (2.54) can be decomposed into the edge adjacent to the root and the two shapes $\alpha_{1} \in \Sigma_{r_{1}}, \alpha_{2} \in \Sigma_{r_{2}}$ emanating from the vertex in $\alpha$ adjacent to the root. We take $\alpha_{1}$ to include the vertex labelled 1 in $\alpha$. By construction, $r_{1}+r_{2}=r+1, r_{1}<r$ and $r_{2}<r$. The integral with respect to $t$ in (2.54) corresponds to integrating out the time variable associated to the edge of $\alpha$ adjacent to the root. The identity (2.54), which shows features analogous to (2.46), will be used in the proof of Proposition 2.3.

Proof of Theorem 1.2 assuming Proposition 2.3. - Let $r \geqslant 3$. The proof is by induction on $r$, with induction hypothesis that (2.52) holds for $\tau^{(s)}$ with $2 \leqslant s<r$. We have seen in (2.53) that (2.52) does hold for $r=2$. The induction will be advanced using (2.46). By Proposition 2.3(ii), $\hat{\varphi}_{\vec{n}}^{(r)}(\vec{k})$ is an error term. Thus, we are left to determine the asymptotic behaviour of the first term on the right side of (2.46).

Fix $\vec{k}$ with $|k|^{2}$ bounded. To abbreviate the notation, we write $\vec{\kappa}=\vec{k} / \sqrt{v \sigma^{2} n}$. Recall the notation $\underline{n}=\min \left\{n_{1}, \ldots, n_{r-1}\right\}$. Given $0 \leqslant n_{0} \leqslant \underline{n}$, let $\underline{n}_{0}=\min \left\{n_{0}, \underline{n}-n_{0}\right\}$. We will show that for every nonempty subset $I \subset J_{1}$,

$$
\begin{aligned}
& \mid \sum_{m_{1}=2}^{\underline{n}_{J \backslash I}-n_{0}} \sum_{m_{2}=2}^{\underline{n}_{I}-n_{0}} \hat{\psi}_{m_{1}, m_{2}}\left(\kappa_{J \backslash I}, \kappa_{I}\right) \hat{\tau}_{\vec{n}_{J \backslash I}-m_{1}-n_{0}}^{\left(r_{1}\right)}\left(\vec{\kappa}_{J \backslash I}\right) \hat{\tau}_{\vec{n}_{I}-m_{2}-n_{0}}^{\left(r_{2}\right)}\left(\vec{\kappa}_{I}\right) \\
& \quad-V \hat{\tau}_{\vec{n}_{J \backslash I}}^{\left(r_{1}\right)}\left(\vec{\kappa}_{J \backslash I}\right) \hat{\tau}_{\vec{n}_{I}-n_{0}}^{\left(r_{2}\right)}\left(\vec{\kappa}_{I}\right) \mid \leqslant n^{r-3}\left(\underline{n}_{0}+1\right)^{-\delta} .
\end{aligned}
$$

Before establishing (2.55), we first show that it implies (2.52). Since $\left|\hat{\tau}_{n_{0}}(\kappa)\right|$ is uniformly bounded by Theorem 1.1(a), inserting (2.55) into (2.46) and applying Proposition 2.3(ii) gives

$$
\begin{aligned}
\hat{\tau}_{\vec{n}}^{(r)}(\vec{\kappa})= & V \sum_{n_{0}=0}^{\underline{n}} \hat{\tau}_{n_{0}}^{(2)}(\kappa) \sum_{I \subset J_{1}:|I| \geqslant 1} \hat{\tau}_{\vec{n}_{J \backslash I}^{\left(r_{1}\right)}}\left(\vec{\kappa}_{J \backslash I}\right) \hat{\tau}_{\vec{n}_{I}-n_{0}}^{\left(r_{2}\right)}\left(\vec{\kappa}_{I}\right) \\
& +\mathcal{O}\left(n^{r-3}\right) \sum_{n_{0}=0}^{\underline{n}}\left(\underline{n}_{0}+1\right)^{-\delta}+\mathcal{O}\left(n^{r-2-\delta}\right) .
\end{aligned}
$$


Using the fact that $\delta<1$, the summation in the error term can be seen to be bounded by a multiple of $\underline{n}^{1-\delta} \leqslant n^{1-\delta}$. With the induction hypothesis and the identity $r_{1}+r_{2}=r+1$, (2.56) then implies that

$$
\begin{aligned}
\hat{\tau}_{\vec{n}}^{(r)}(\vec{\kappa})= & V A^{3}\left(A^{2} V n\right)^{r-3} \sum_{n_{0}=0}^{\underline{n}} \widehat{M}_{n_{0} / n}^{(1)}(k) \\
& \times \sum_{I \subset J_{1}:|I| \geqslant 1} \widehat{M}_{\left(\bar{n}_{J \backslash I}-n_{0}\right) / n}^{\left(r_{1}-1\right)}\left(\vec{k}_{J \backslash I}\right) \widehat{M}_{\left(\vec{n}_{I}-n_{0}\right) / n}^{\left(r_{2}-1\right)}\left(\vec{k}_{I}\right)+\mathcal{O}\left(n^{r-2-\delta}\right),
\end{aligned}
$$

where the error arising from the error terms in the induction hypothesis again contributes an amount $\mathcal{O}\left(n^{r-3}\right) \sum_{n_{0}=0}^{\underline{n}}\left(\underline{n}_{0}+1\right)^{-\delta} \leqslant \mathcal{O}\left(n^{r-2-\delta}\right)$. The summation on the right side of (2.57), divided by $n$, is the Riemann sum approximation to an integral. The error in approximating the integral by this Riemann sum is $\mathcal{O}\left(n^{-1}\right)$. Therefore, using (2.54), we obtain

$$
\begin{aligned}
\hat{\tau}_{\vec{n}}^{(r)}(\vec{\kappa})= & A\left(A^{2} V n\right)^{r-2} \int_{0}^{n / n} \mathrm{~d} t \widehat{M}_{t}^{(1)}(k) \\
& \times \sum_{I \subset J:|I| \geqslant 1} \widehat{M}_{n^{-1} \vec{n}_{J \backslash I}-t}^{\left(r_{1}-1\right)}\left(\vec{k}_{J \backslash I}\right) \widehat{M}_{n^{-1} \vec{n}_{I}-t}^{\left(r_{2}-1\right)}\left(\vec{k}_{I}\right)+\mathcal{O}\left(n^{r-2-\delta}\right) \\
= & A\left(A^{2} V n\right)^{r-2} \widehat{M}_{\vec{n} / n}^{(r-1)}(\vec{k})+\mathcal{O}\left(n^{r-2-\delta}\right) .
\end{aligned}
$$

Since $n \geqslant \bar{n}$, it follows that $n^{r-2-\delta} \leqslant C n^{r-2}(\bar{n}+1)^{-\delta}$. Thus, it suffices to establish (2.55). To prove (2.55), we write the quantity inside the absolute value signs on the left side as

$$
T_{1}+T_{2}+T_{3}
$$

with

$$
\begin{aligned}
T_{1}= & \left(\sum_{m_{1}=2}^{\underline{n}_{J I I}-n_{0}} \sum_{m_{2}=2}^{\underline{n}_{I}-n_{0}} \hat{\psi}_{m_{1}, m_{2}}(0,0)-V\right) \hat{\tau}_{\vec{n}_{J \backslash I}-n_{0}}^{\left(r_{1}\right)}\left(\vec{\kappa}_{J \backslash I}\right) \hat{\tau}_{\vec{n}_{I}-n_{0}}^{\left(r_{2}\right)}\left(\vec{\kappa}_{I}\right), \\
T_{2}= & \sum_{m_{1}=2}^{\underline{n}_{J \backslash I}-n_{0}} \sum_{m_{2}=2}^{\underline{n}_{I}-n_{0}}\left(\hat{\psi}_{m_{1}, m_{2}}\left(\kappa_{J \backslash I}, \kappa_{I}\right)-\hat{\psi}_{m_{1}, m_{2}}(0,0)\right) \\
& \times \hat{\tau}_{\vec{n}_{J \backslash I}^{\left(r_{1}\right)}}\left(\vec{\kappa}_{J \backslash I}\right) \hat{\tau}_{\vec{n}_{I}-n_{0}}^{\left(r_{2}\right)}\left(\vec{\kappa}_{I}\right), \\
T_{3}= & \sum_{m_{1}=2}^{\underline{n}_{J \backslash I}-n_{0}} \sum_{m_{2}=2}^{\underline{n}_{I}-n_{0}} \hat{\psi}_{m_{1}, m_{2}}\left(\kappa_{J \backslash I}, \kappa_{I}\right) \\
& \times\left(\hat{\tau}_{\left.\vec{n}_{J \backslash I}-r_{1}\right)}^{\left(r_{1}\right)}\left(\vec{\kappa}_{J \backslash I}\right) \hat{\tau}_{\vec{n}_{I}-m_{2}-n_{0}}^{\left(r_{2}\right)}\left(\vec{\kappa}_{I}\right)-\hat{\tau}_{\vec{n}_{J \backslash I}-n_{0}}^{\left(r_{1}\right)}\left(\vec{\kappa}_{J \backslash I}\right) \hat{\tau}_{\vec{n}_{I}-n_{0}}^{\left(r_{2}\right)}\left(\vec{\kappa}_{I}\right)\right) .
\end{aligned}
$$

To complete the proof, it suffices to show that for each nonempty $I \subset J_{1}$, the absolute value of each $T_{i}$ is bounded above by the right side of (2.55).

By the induction hypothesis and the fact that $\bar{n}_{I_{i}} \leqslant n$, it follows that $\left|\hat{\tau}_{\bar{n}_{I_{i}}}^{\left(r_{i}\right)}\left(\vec{k}_{I_{i}}\right)\right| \leqslant$ $\mathcal{O}\left(n^{r_{i}-2}\right)$, uniformly in $\vec{n}_{I_{i}}$ and $\vec{k}_{I_{i}}$. Therefore, it follows from Proposition 2.3(i) and the 
definition of $V$ in (2.49) that

$$
\left|T_{1}\right| \leqslant \sum_{\substack{m_{1} \geqslant \underline{n}_{J \backslash I}-n_{0} \\ \text { or } m_{2} \geqslant \underline{n}_{I}-n_{0}}} \frac{\mathcal{O}\left(n^{r-3}\right)}{\left(m_{1} \vee m_{2}+1\right)^{d / 2}} \leqslant \mathcal{O}\left(n^{r-3}\left(\underline{n}_{0}+1\right)^{-(d-4) / 2}\right) .
$$

Similarly, by Proposition 2.3(i) with $q=2$, a second-order Taylor estimate gives

$$
\left|T_{2}\right| \leqslant \sum_{m_{1}=2}^{\underline{n}_{J \backslash I}-n_{0}} \sum_{m_{2}=2}^{\underline{n}_{I}-n_{0}}\left(m_{1}\left|\kappa_{J \backslash I}\right|^{2}+m_{2}\left|\kappa_{I}\right|^{2}\right) \frac{\mathcal{O}\left(n^{r-3}\right)}{\left(m_{1} \vee m_{2}+1\right)^{d / 2}} \leqslant \mathcal{O}\left(n^{r-3}\left(\underline{n}_{0}+1\right)^{-\delta}\right) .
$$

It remains to prove that

$$
\left|T_{3}\right| \leqslant \mathcal{O}\left(n^{r-3}\left(\underline{n}_{0}+1\right)^{-\delta}\right) .
$$

To begin the proof of (2.65), we note that the domain of summation over $m_{1}, m_{2}$ in (2.62) is contained in $\bigcup_{j=0}^{2} \mathcal{S}_{j}(\vec{n})$, where

$$
\begin{aligned}
& \mathcal{S}_{0}(\vec{n})=\left[0, \frac{1}{2}\left(\underline{n}_{J \backslash I}-n_{0}\right)\right] \times\left[0, \frac{1}{2}\left(\underline{n}_{I}-n_{0}\right)\right], \\
& \mathcal{S}_{1}(\vec{n})=\left[\frac{1}{2}\left(\underline{n}_{J \backslash I}-n_{0}\right), \underline{n}_{J \backslash I}-n_{0}\right] \times\left[0, \underline{n}_{I}-n_{0}\right], \\
& \mathcal{S}_{2}(\vec{n})=\left[0, \underline{n}_{J \backslash I}-n_{0}\right] \times\left[\frac{1}{2}\left(\underline{n}_{I}-n_{0}\right), \underline{n}_{I}-n_{0}\right] .
\end{aligned}
$$

Therefore $\left|T_{3}\right|$ is bounded by

$$
\begin{aligned}
& \sum_{j=0}^{2} \sum_{\vec{m} \in \mathcal{S}_{j}(\vec{n})}\left|\hat{\psi}_{m_{1}, m_{2}}\left(\kappa_{J \backslash I}, \kappa_{I}\right)\right| \\
& \quad \times\left|\hat{\tau}_{\vec{n}_{J \backslash I}^{\left(r_{1}\right)}-m_{1}-n_{0}}\left(\vec{\kappa}_{J \backslash I}\right) \hat{\tau}_{\vec{n}_{I}-m_{2}-n_{0}}^{\left(r_{2}\right)}\left(\vec{\kappa}_{I}\right)-\hat{\tau}_{\vec{n}_{J \backslash I}-n_{0}}^{\left(r_{1}\right)}\left(\vec{\kappa}_{J \backslash I}\right) \hat{\tau}_{\vec{n}_{I}-n_{0}}^{\left(r_{2}\right)}\left(\vec{\kappa}_{I}\right)\right| .
\end{aligned}
$$

The terms with $j=1,2$ in (2.66) can be estimated as in the bound (2.63) on $T_{1}$, after using the triangle inequality and bounding the $r_{i}$-point functions by $\mathcal{O}\left(n^{r_{i}-2}\right)$.

For the $j=0$ term of (2.66), we write

$$
\begin{aligned}
& \hat{\tau}_{\vec{n}_{J \backslash I}\left(r_{1}\right) m_{1}-n_{0}}\left(\vec{\kappa}_{J \backslash I}\right)=\hat{\tau}_{\vec{n}_{J \backslash I}\left(r_{1}\right)}^{\left(r_{0}\right.}\left(\vec{\kappa}_{J \backslash I}\right)+\left[\hat{\tau}_{\vec{n}_{J \backslash I}\left(r_{1}\right)}{ }_{m_{1}-n_{0}}\left(\vec{\kappa}_{J \backslash I}\right)-\hat{\tau}_{\vec{n}_{J \backslash I}\left(r_{1}\right)}\left(\vec{\kappa}_{J \backslash I}\right)\right], \\
& \hat{\tau}_{\vec{n}_{I}-m_{2}-n_{0}}^{\left(r_{2}\right)}\left(\vec{\kappa}_{I}\right)=\hat{\tau}_{\vec{n}_{I}-n_{0}}^{\left(r_{2}\right)}\left(\vec{\kappa}_{I}\right)+\left[\hat{\tau}_{\vec{n}_{I}-m_{2}-n_{0}}^{\left(r_{2}\right)}\left(\vec{\kappa}_{I}\right)-\hat{\tau}_{\vec{n}_{I}-n_{0}}^{\left(r_{2}\right)}\left(\vec{\kappa}_{I}\right)\right] .
\end{aligned}
$$

We expand the product of (2.67) and (2.68). This gives four terms, one of which is cancelled by $\hat{\tau}_{\vec{n}_{J \backslash I}-n_{0}}^{\left(r_{1}\right)}\left(\vec{\kappa}_{J \backslash I}\right) \hat{\tau}_{\vec{n}_{I}-n_{0}}^{\left(r_{2}\right)}\left(\vec{\kappa}_{I}\right)$ in (2.66). Three terms remain, each of which contains at least one factor from the second terms in (2.67) and (2.68). In each term we retain one such factor and bound the other factor by a power of $n$, and we estimate $\hat{\psi}$ using Proposition 2.3(i). This gives a bound for the $j=0$ contribution to (2.66) equal to the sum of

$$
\sum_{\left(m_{1}, m_{2}\right) \in \mathcal{S}_{0}(\vec{n})} \frac{\mathcal{O}\left(n^{r_{2}-2}\right)}{\left(m_{1} \vee m_{2}+1\right)^{d / 2}}\left|\hat{\tau}_{\vec{n}_{J \backslash I}^{\left(r_{1}\right)}}\left(\vec{\kappa}_{J \backslash I}\right)-\hat{\tau}_{\vec{n}_{J \backslash I}-n_{0}}^{\left(r_{1}\right)}\left(\vec{\kappa}_{J \backslash I}\right)\right|
$$


plus a similar term with $J \backslash I$ replaced by $I$. By the induction hypothesis, the difference of $r_{1}$-point functions in (2.69) is equal to

$$
A\left(A^{2} V n\right)^{r_{1}-2}\left[f\left(\left(\vec{n}_{J \backslash I}-m_{1}-n_{0}\right) / n\right)-f\left(\left(\vec{n}_{J \backslash I}-n_{0}\right) / n\right)\right]+\mathcal{O}\left(n^{r_{1}-2}\left(\underline{n}_{0}+1\right)^{-\delta}\right)
$$

with $f(\vec{t})=\widehat{M}_{\vec{t}}^{\left(r_{1}-1\right)}\left(\vec{k}_{J \backslash I}\right)$. Using (2.51), the difference in (2.70) can be seen to be at most $\mathcal{O}\left(m_{1} n^{-1}\right)$. Therefore (2.69) is bounded above by

$$
\sum_{\left(m_{1}, m_{2}\right) \in \mathcal{S}_{0}(\vec{n})} \frac{\mathcal{O}\left(n^{r-4}\right)}{\left(m_{1} \vee m_{2}+1\right)^{(d-2) / 2}}+\mathcal{O}\left(n^{r-3}\left(\underline{n}_{0}+1\right)^{-\delta}\right) \leqslant \mathcal{O}\left(n^{r-3}\left(\underline{n}_{0}+1\right)^{-\delta}\right) .
$$

This establishes (2.65).

Combining (2.63)-(2.65) yields (2.55). This completes the proof of Theorem 1.2, assuming Proposition 2.3.

\section{Expansion for the two-point function}

There are two possible expansions available for oriented percolation. The NguyenYang expansion, which is close in spirit to the original lace expansion of Brydges and Spencer [8], was used in [31,32] to study the critical oriented percolation two-point function in high dimensions. We are extending the results of Nguyen and Yang in this paper. The Nguyen-Yang expansion relies on a Markov property valid for oriented percolation (but not for non-oriented percolation). The Hara-Slade expansion [20] is an alternate expansion, based on inclusion-exclusion rather than using the notion of "laces". It was derived in the context of non-oriented percolation, but it applies to oriented percolation without alteration.

Both expansions lead to the same quantity $\pi_{n}(x)$, obeying the same identity (2.5). However, the expansions lead to different decompositions of $\pi_{n}(x)$ into alternating series of the form $\sum_{N=0}^{\infty}(-1)^{N} \pi_{n}^{(N)}(x)$. In each expansion, the $\pi_{n}^{(N)}(x)$ are described by Feynman diagrams. The diagrams for the two expansions are almost identical, and they obey identical upper bounds (see Section 4.4). However, the precise definition of the $\pi_{n}^{(N)}(x)$ is different according to which expansion is used. In the Hara-Slade expansion, $\pi_{n}^{(N)}(x)$ is given by an $(N+1)$-fold nested expectation, whereas the Nguyen-Yang expansion involves only a single expectation for each $N$. In this respect, the NguyenYang expansion is simpler.

We will mainly employ the Hara-Slade expansion, since it can be extended from the two-point function to general $r$-point functions, as we will describe in Sections 5-6. This seems simpler to us than attempting to extend the Nguyen-Yang expansion to general $r$-point functions, as the use of the Markov property becomes problematic for $r \geqslant 3$. In particular, the use of the Markov property seems well suited to extraction of the twopoint function mentioned in item (1) of Section 2.2.1, but the two branches mentioned in items (3) and (4) coexist in the same temporal domain and it is unclear to us how the Nguyen-Yang expansion might be modified to allow for their decoupling.

However, we will make use of the Nguyen-Yang expansion in proving the bound on $p \partial_{p} \hat{\pi}_{m}(0)$ of Proposition 2.2. This is because the $p$-dependence is simpler within 
the single expectation of the Nguyen-Yang expansion, so that its derivative is easier to analyse.

Our approach to the two-point function differs from that of Nguyen and Yang not just in the use of a different expansion. Whereas Nguyen and Yang proved convergence of the lace expansion using generating functions, we will instead apply the inductive approach of [27], which generalises the method of [25]. Implementation of the induction method was described in Section 2.1.2. The induction method avoids the necessity of extracting the asymptotic behaviour of a sequence from the singularity structure of its generating function. It also provides the bound on $\left\|\tau_{n}\right\|_{\infty}$ of Theorem 1.1(c), which will be needed in our analysis of the $r$-point functions for $r \geqslant 3$.

In the remainder of this section, we will derive the two expansions for the two-point function.

\subsection{The Hara-Slade expansion}

In this section, we derive the expansion (2.5) for the two-point function, using the method of [20]. Although the derivation presented here is essentially identical to that of [20], we include it both to make this paper more self-contained and to allow for reference later when we derive the expansion for the $r$-point functions in Section 5.

The expansion produces a function $\pi_{m}(x)$ such that

$$
\tau_{n}(x)=p\left(D * \tau_{n-1}\right)(x)+\sum_{m=2}^{n-1} p\left(\pi_{m} * D * \tau_{n-m-1}\right)(x)+\pi_{n}(x) \quad(n \geqslant 1),
$$

which is just (2.5). We will use the notation $\boldsymbol{x}=(x, n)$ introduced in Section 2.2. In addition, we will use $(\boldsymbol{u}, \boldsymbol{v})$ as a summation index when it is guaranteed that the temporal component of $\boldsymbol{v}$ exceeds that of $\boldsymbol{u}$ by exactly 1 . In this notation, (3.1) becomes

$$
\tau(\boldsymbol{x})=\delta_{\mathbf{0}, \boldsymbol{x}}+\sum_{(\mathbf{0}, \boldsymbol{u})} p D(\boldsymbol{u}) \tau(\boldsymbol{x}-\boldsymbol{u})+p \sum_{(\boldsymbol{u}, \boldsymbol{v})} \pi(\boldsymbol{u}) D(\boldsymbol{v}-\boldsymbol{u}) \tau(\boldsymbol{x}-\boldsymbol{v})+\pi(\boldsymbol{x})
$$

with $D(\boldsymbol{x})$ given by (2.2). The function $\pi(\boldsymbol{x})=\pi_{n}(x)$ will be written in terms of functions $\pi^{(N)}(\boldsymbol{x})$ as

$$
\pi(\boldsymbol{x})=\sum_{N=0}^{\infty}(-1)^{N} \pi^{(N)}(\boldsymbol{x}) .
$$

To begin the expansion, we make three definitions which underlie the string-of-sausage image of Fig. 2.

Definition 3.1. - (a) Given a configuration and $\boldsymbol{x} \in \Lambda$ we define $C(\boldsymbol{x})=\{\boldsymbol{y} \in$ $\Lambda: \boldsymbol{x} \rightarrow \boldsymbol{y}\}$.

(b) Given a configuration, we say that $\boldsymbol{x}$ is doubly connected to $\boldsymbol{y}$, and we write $\boldsymbol{x} \Rightarrow \boldsymbol{y}$, if there are at least two bond-disjoint paths from $\boldsymbol{x}$ to $\boldsymbol{y}$ consisting of occupied bonds. By convention, we say that $\boldsymbol{x} \Rightarrow \boldsymbol{x}$ for all $\boldsymbol{x}$.

(c) Given a configuration, we say that a bond is pivotal for $\boldsymbol{x} \rightarrow \boldsymbol{y}$ if $\boldsymbol{x} \rightarrow \boldsymbol{y}$ in the possibly modified configuration in which the bond is made occupied, whereas $\boldsymbol{x}$ is not connected to $\boldsymbol{y}$ in the possibly modified configuration in which the bond is made vacant. 
To begin the expansion, we define

$$
\pi^{(0)}(\boldsymbol{x})=\mathbb{P}(\mathbf{0} \Rightarrow \boldsymbol{x})-\delta_{\mathbf{0}, \boldsymbol{x}}
$$

and distinguish configurations with $\mathbf{0} \rightarrow \boldsymbol{x}$ according to whether or not there is a double connection, to obtain

$$
\tau(\boldsymbol{x})=\delta_{\mathbf{0}, \boldsymbol{x}}+\pi^{(0)}(\boldsymbol{x})+\mathbb{P}(\mathbf{0} \rightarrow \boldsymbol{x} \& \mathbf{0} \not \boldsymbol{x})
$$

If $\mathbf{0}$ is connected to $\boldsymbol{x}$, but not doubly, then there is at least one pivotal bond for the connection, and hence a first such pivotal bond. Denoting this pivotal bond by $(\boldsymbol{u}, \boldsymbol{v})$, we can write

$$
\mathbb{P}(\mathbf{0} \rightarrow \boldsymbol{x} \& \mathbf{0} \not \boldsymbol{x})=\sum_{(\boldsymbol{u}, \boldsymbol{v})} \mathbb{P}(\mathbf{0} \Rightarrow \boldsymbol{u} \text { and }(\boldsymbol{u}, \boldsymbol{v}) \text { is occupied and pivotal for } \mathbf{0} \rightarrow \boldsymbol{x})
$$

Now comes the essential part of the expansion. Ideally, we would like to factor the probability on the right side of (3.6) as

$$
\mathbb{P}(\mathbf{0} \Rightarrow \boldsymbol{u}) \mathbb{P}((\boldsymbol{u}, \boldsymbol{v}) \text { is occupied }) \mathbb{P}(\boldsymbol{v} \rightarrow \boldsymbol{x})=\left(\delta_{\mathbf{0}, \boldsymbol{x}}+\pi^{(0)}(\boldsymbol{u})\right) p D(\boldsymbol{u}-\boldsymbol{v}) \tau(\boldsymbol{x}-\boldsymbol{v})
$$

However, it does not factor in this way because the sausage connecting $\mathbf{0}$ to $\boldsymbol{u}$ is constrained not to send out a branch that would intersect the portion of the cluster after $\boldsymbol{v}$ in such a way as to contradict the pivotal nature of the bond $(\boldsymbol{u}, \boldsymbol{v})$. What we can do is approximate the probability on the right side of (3.6) by (3.7), and then attempt to deal with the error term. For this purpose, we need several more definitions.

DEFINITION 3.2. - (a) Given a bond configuration, and $A \subset \Lambda$, we say $\boldsymbol{x}$ and $\boldsymbol{y}$ are connected in $A$, if there is an occupied path from $\boldsymbol{x}$ to $\boldsymbol{y}$ having all its endpoints in $A$, or if $\boldsymbol{x}=\boldsymbol{y} \in A$. We define a restricted two-point function by

$$
\tau^{A}(\boldsymbol{x}, \boldsymbol{y})=\mathbb{P}(\boldsymbol{x} \text { and } \boldsymbol{y} \text { are connected in } \Lambda \backslash A) .
$$

(b) Given a bond configuration, and $A \subset \Lambda$, we say $\boldsymbol{x}$ and $\boldsymbol{y}$ are connected through $A$, if every occupied path connecting $\boldsymbol{x}$ to $\boldsymbol{y}$ has at least one bond with an endpoint in $A$. This event is written as $\boldsymbol{x} \stackrel{A}{\rightarrow} \boldsymbol{y}$.

(c) Given a bond configuration, and a bond $b$, we define $\widetilde{C}^{b}(\boldsymbol{x})$ to be the set of sites $\boldsymbol{y}$ such that $\boldsymbol{x}$ is connected to $\boldsymbol{y}$ in the new configuration obtained by setting $b$ to be vacant.

(d) Given an event $E$, we define the event $\left\{E\right.$ occurs on $\left.\widetilde{C}^{(\boldsymbol{u}, \boldsymbol{v})}(\boldsymbol{x})\right\}$ to be the set of configurations such that $E$ occurs on the modified configuration in which every bond that does not have an endpoint in $\widetilde{C}^{(\boldsymbol{u}, \boldsymbol{v})}(\boldsymbol{x})$ is made vacant. We say that $\{$ E occurs in $\left.\Lambda \backslash \widetilde{C}^{(\boldsymbol{u}, \boldsymbol{v})}(\boldsymbol{x})\right\}$ if $E$ occurs on the modified configuration in which every bond that does not have both endpoints in $\Lambda \backslash \widetilde{\boldsymbol{C}}^{(\boldsymbol{u}, \boldsymbol{v})}(\boldsymbol{x})$ is made vacant.

In terms of these definitions, we have the following important lemma, which is essentially [22, Lemma 2.4]. 
LemMA 3.3. - Fix $p \in[0,1]$. Given a bond $(\boldsymbol{u}, \boldsymbol{v})$, a site $\boldsymbol{w}$ and events $E, F$ determined by the occupation status of finitely many bonds,

$$
\begin{aligned}
& \mathbb{E}_{p}\left(I\left[E \text { occurs on } \widetilde{C}^{(\boldsymbol{u}, \boldsymbol{v})}(\boldsymbol{w}) \&(\boldsymbol{u}, \boldsymbol{v}) \text { is occupied \& } F \text { occurs in } \Lambda \backslash \widetilde{C}^{(\boldsymbol{u}, \boldsymbol{v})}(\boldsymbol{w})\right]\right) \\
& \quad=p D(\boldsymbol{v}-\boldsymbol{u}) \mathbb{E}_{p}\left(I\left[E \text { occurs on } \widetilde{C}^{(\boldsymbol{u}, \boldsymbol{v})}(\boldsymbol{w})\right] \mathbb{E}_{p}\left(I\left[F \operatorname{occurs} \text { in } \Lambda \backslash \widetilde{C}^{(\boldsymbol{u}, \boldsymbol{v})}(\boldsymbol{w})\right]\right)\right) .
\end{aligned}
$$

We omit the proof of Lemma 3.3, as it is a small modification of the proof in [22]. The lemma is proved by conditioning on the restriction of $\widetilde{C}^{(\boldsymbol{u}, \boldsymbol{v})}(\boldsymbol{w})$ to a finite time horizon in $\Lambda$ that is sufficient to include all bonds on which $E$ and $F$ depend. This ensures that we condition on a finite cluster, and we therefore do not need the restriction $p \leqslant p_{c}$ of [22, Lemma 2.4].

In the nested expectation on the right side, the set $\widetilde{C}^{(\boldsymbol{u}, \boldsymbol{v})}(\boldsymbol{w})$ is a random set with respect to the outer expectation, but it is deterministic with respect to the inner expectation. The inner expectation on the right side effectively introduces a second percolation model on a second lattice, which is coupled to the original percolation model via the set $\widetilde{C}^{(\boldsymbol{u}, \boldsymbol{v})}(\boldsymbol{w})$.

To apply Lemma 3.3, we first note that

$$
\begin{gathered}
\{\boldsymbol{0} \Rightarrow \boldsymbol{u} \text { and }(\boldsymbol{u}, \boldsymbol{v}) \text { is occupied and pivotal for } \mathbf{0} \rightarrow \boldsymbol{x}\} \\
=\left\{\boldsymbol{0} \Rightarrow \boldsymbol{u} \text { occurs on } \widetilde{C}^{(\boldsymbol{u}, \boldsymbol{v})}(\mathbf{0}) \&(\boldsymbol{u}, \boldsymbol{v})\right. \text { is occupied } \\
\left.\& \boldsymbol{v} \rightarrow \boldsymbol{x} \text { occurs in } \Lambda \backslash \widetilde{C}^{(\boldsymbol{u}, \boldsymbol{v})}(\mathbf{0})\right\} .
\end{gathered}
$$

The identity (3.10) is slightly more delicate than its non-oriented counterpart, due to the fact that it is possible in the oriented context for the clusters $\widetilde{C}^{(\boldsymbol{u}, \boldsymbol{v})}(\mathbf{0})$ and $\widetilde{C}^{(\boldsymbol{u}, \boldsymbol{v})}(\boldsymbol{v})$ to intersect each other even when $(\boldsymbol{u}, \boldsymbol{v})$ is pivotal for $\mathbf{0} \rightarrow \boldsymbol{x}$. To prove (3.10), we first observe that the event on the left side implies that $\mathbf{0} \Rightarrow \boldsymbol{u}$ occurs on $\widetilde{C}^{(\boldsymbol{u}, \boldsymbol{v})}(\mathbf{0})$, that $(\boldsymbol{u}, \boldsymbol{v})$ is occupied, and that $\boldsymbol{v} \rightarrow \boldsymbol{x}$. To see that $\boldsymbol{v}$ is connected to $\boldsymbol{x}$ in $\Lambda \backslash \widetilde{C}^{(\boldsymbol{u}, \boldsymbol{v})}(\mathbf{0})$, suppose to the contrary that every occupied path from $v$ to $x$ contains a site $z \in \widetilde{C}^{(\boldsymbol{u}, \boldsymbol{v})}(\mathbf{0})$ (which may depend on the path). Then $\mathbf{0}$ is connected to $\boldsymbol{x}$ via $\boldsymbol{z}$ even after $(\boldsymbol{u}, \boldsymbol{v})$ is made vacant, contradicting the pivotal nature of $(\boldsymbol{u}, \boldsymbol{v})$. Conversely, the event on the right side of (3.10) implies that $\mathbf{0} \Rightarrow \boldsymbol{u}$, that $(\boldsymbol{u}, \boldsymbol{v})$ is occupied and that $\mathbf{0} \rightarrow \boldsymbol{x}$. To see that $(\boldsymbol{u}, \boldsymbol{v})$ must be pivotal, we observe that if it is not then $\boldsymbol{x} \in \widetilde{C}^{(\boldsymbol{u}, \boldsymbol{v})}(\mathbf{0})$ and it cannot be the case that $\boldsymbol{v} \rightarrow \boldsymbol{x}$ in $\Lambda \backslash \widetilde{C}^{(\boldsymbol{u}, \boldsymbol{v})}(\mathbf{0})$. This proves (3.10).

By Lemma 3.3, (3.10) gives the important identity

$$
\begin{aligned}
\mathbb{P}(\mathbf{0} & \Rightarrow \boldsymbol{u} \text { and }(\boldsymbol{u}, \boldsymbol{v}) \text { is occupied and pivotal for } \mathbf{0} \rightarrow \boldsymbol{x}) \\
& =p D(\boldsymbol{v}-\boldsymbol{u}) \mathbb{E}\left(I\left[\mathbf{0} \Rightarrow \boldsymbol{u} \text { occurs on } \widetilde{C}^{(\boldsymbol{u}, \boldsymbol{v})}(\mathbf{0})\right] \tau^{\widetilde{C}^{(\boldsymbol{u}, \boldsymbol{v})}(\mathbf{0})}(\boldsymbol{v}, \boldsymbol{x})\right) \\
& =p D(\boldsymbol{v}-\boldsymbol{u}) \mathbb{E}\left(I[\mathbf{0} \Rightarrow \boldsymbol{u}] \tau^{\widetilde{C}^{(\boldsymbol{u}, \boldsymbol{v})}(\boldsymbol{0})}(\boldsymbol{v}, \boldsymbol{x})\right),
\end{aligned}
$$

where we can drop the condition "occurs on $\widetilde{C}^{(\boldsymbol{u}, \boldsymbol{v})}(\mathbf{0})$ " since $\mathbf{0} \Rightarrow \boldsymbol{u}$ is independent of the occupation status of the bond $(\boldsymbol{u}, \boldsymbol{v})$, due to the orientation. On the right side, ${ }_{\tau^{C^{(u, v)}}(\boldsymbol{0})}(\boldsymbol{v}, \boldsymbol{x})$ is the restricted two-point function given the cluster $\widetilde{C}^{(\boldsymbol{u}, \boldsymbol{v})}(\mathbf{0})$ of the outer expectation, so that in the (inner) expectation defining $\tau^{\widetilde{C}^{(u, v)}(\mathbf{0})}(\boldsymbol{v}, \boldsymbol{x}), \widetilde{C}^{(\boldsymbol{u}, \boldsymbol{v})}(\mathbf{0})$ should be regarded as a fixed set. We stress this delicate point here, as it is crucial also in the rest 
of the expansion. As mentioned above, we should think of configurations corresponding to the expectation defining $\tau^{\widetilde{C}^{(u, v)}(\boldsymbol{0})}(\boldsymbol{v}, \boldsymbol{x})$ as living on a different lattice than $\widetilde{C}^{(\boldsymbol{u}, \boldsymbol{v})}(\mathbf{0})$.

It follows from (3.6) and (3.11) that

$$
\mathbb{P}(\mathbf{0} \rightarrow \boldsymbol{x} \& \mathbf{0} \nRightarrow \boldsymbol{x})=\sum_{(\boldsymbol{u}, \boldsymbol{v})} p D(\boldsymbol{v}-\boldsymbol{u}) \mathbb{E}\left(I[\mathbf{0} \Rightarrow \boldsymbol{u}] \tau^{\widetilde{C}^{(\boldsymbol{u}, v)}(\mathbf{0})}(\boldsymbol{v}, \boldsymbol{x})\right) .
$$

We write

$$
\begin{aligned}
\tau^{\widetilde{C}^{(u, v)}(\boldsymbol{0})}(\boldsymbol{v}, \boldsymbol{x}) & =\tau(\boldsymbol{x}-\boldsymbol{v})-\left(\tau(\boldsymbol{x}-\boldsymbol{v})-\tau^{\widetilde{C}^{(u, v)}(\boldsymbol{0})}(\boldsymbol{v}, \boldsymbol{x})\right) \\
& =\tau(\boldsymbol{x}-\boldsymbol{v})-\mathbb{P}\left(\boldsymbol{v} \stackrel{\widetilde{C}^{(u, v)}(\mathbf{0})}{\longrightarrow} \boldsymbol{x}\right),
\end{aligned}
$$

insert this into (3.12), and use (3.5) and (3.4) to obtain

$$
\begin{aligned}
\tau(\boldsymbol{x})= & \delta_{\boldsymbol{0}, \boldsymbol{x}}+\pi^{(0)}(\boldsymbol{x})+\sum_{(\boldsymbol{u}, \boldsymbol{v})}\left(\delta_{\mathbf{0}, \boldsymbol{u}}+\pi^{(0)}(\boldsymbol{u})\right) p D(\boldsymbol{v}-\boldsymbol{u}) \tau(\boldsymbol{x}-\boldsymbol{v}) \\
& -\sum_{(\boldsymbol{u}, \boldsymbol{v})} p D(\boldsymbol{v}-\boldsymbol{u}) \mathbb{E}\left(I[\mathbf{0} \Rightarrow \boldsymbol{u}] \mathbb{P}\left(\boldsymbol{v} \stackrel{\widetilde{C}^{(\boldsymbol{u}, \boldsymbol{v})}(\mathbf{0})}{\longrightarrow} \boldsymbol{x}\right)\right) .
\end{aligned}
$$

This completes the first stage of the expansion.

Before proceeding with the expansion, we take stock of what has been achieved so far. If we were to neglect the final term in (3.14), we would have an equation analogous to (2.4), with $\left(\delta_{0, x}+\pi^{(0)}(\boldsymbol{u})\right)$ corresponding to the weight of a single "site" along a generalised random walk. We expect the final term of (3.14) to be small, provided the backbone joining $\boldsymbol{v}$ to $\boldsymbol{x}$ typically does not intersect the cluster $\widetilde{C}^{(\boldsymbol{u}, \boldsymbol{v})}(\mathbf{0})$. Above the upper critical dimension, the backbone should have the character of Brownian motion and the cluster $\widetilde{C}^{(\boldsymbol{u}, \boldsymbol{v})}(\mathbf{0})$ should have the character of a super-Brownian motion. For oriented percolation, intersection of the backbone and cluster corresponds to intersection of the graphs of Brownian motion and super-Brownian motion. As explained near the end of Section 1.2.3, the critical dimension for such an intersection is $d=4$.

To continue the expansion, we would like to rewrite the final term of (2.4) in terms of a convolution with the two-point function. A configuration contributing to the expectation in the final term of (2.4) is illustrated schematically in Fig. 5, in which the bonds drawn with heavy lines should be regarded as living on a different lattice than the bonds drawn with lighter lines, as explained previously. Our goal is to extract a factor $\tau\left(\boldsymbol{x}-\boldsymbol{v}^{\prime}\right)$.

Given a configuration in which $\boldsymbol{v} \stackrel{A}{\rightarrow} \boldsymbol{x}$, the cutting bond $\left(\boldsymbol{u}^{\prime}, \boldsymbol{v}^{\prime}\right)$ is defined to be the first pivotal bond for $\boldsymbol{v} \rightarrow \boldsymbol{x}$ such that $\boldsymbol{v} \stackrel{A}{\rightarrow} \boldsymbol{u}^{\prime}$. It is possible that no such bond exists, as for example would be the case in Fig. 5 if only the bottom four sausages were included in the figure, with $\boldsymbol{x}$ in the location currently occupied by $\boldsymbol{u}^{\prime}$. To analyse $\mathbb{P}\left(\boldsymbol{v} \stackrel{\widetilde{C}^{(u, v)}(\mathbf{0})}{\longrightarrow} \boldsymbol{x}\right)$, we define the events

$$
\begin{aligned}
& E^{\prime}(\boldsymbol{v}, \boldsymbol{x} ; A)=\{\boldsymbol{v} \stackrel{A}{\rightarrow} \boldsymbol{x}\} \cap\left\{\nexists \text { pivotal }\left(\boldsymbol{u}^{\prime}, \boldsymbol{v}^{\prime}\right) \text { for } \boldsymbol{v} \rightarrow \boldsymbol{x} \text { s.t. } \boldsymbol{v} \stackrel{A}{\rightarrow} \boldsymbol{u}^{\prime}\right\} \\
& E\left(\boldsymbol{v}, \boldsymbol{u}^{\prime}, \boldsymbol{v}^{\prime}, \boldsymbol{x} ; A\right)=E^{\prime}\left(\boldsymbol{v}, \boldsymbol{u}^{\prime} ; A\right) \cap\left\{\left(\boldsymbol{u}^{\prime}, \boldsymbol{v}^{\prime}\right) \text { is occupied and pivotal for } \boldsymbol{v} \rightarrow \boldsymbol{x}\right\}
\end{aligned}
$$




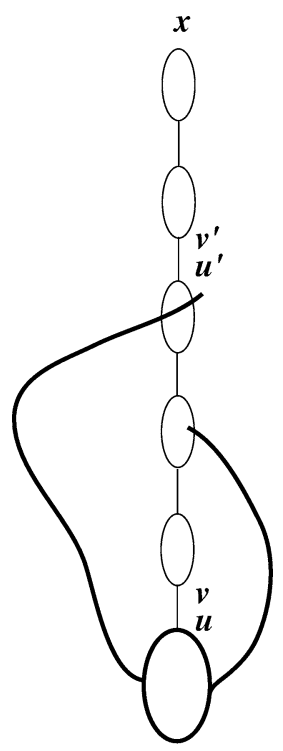

0

Fig. 5. A configuration appearing in the second stage of the expansion, with the heavy and light lines corresponding to percolation clusters living on distinct copies of the same lattice.

By partitioning $\{\boldsymbol{v} \stackrel{A}{\rightarrow} \boldsymbol{x}\}$ according to the location of the cutting bond (or the lack of a cutting bond), we obtain the partition

$$
\{\boldsymbol{v} \stackrel{A}{\rightarrow} \boldsymbol{x}\}=E^{\prime}(\boldsymbol{v}, \boldsymbol{x} ; A) \bigcup_{\left(\boldsymbol{u}^{\prime}, \boldsymbol{v}^{\prime}\right)} E\left(\boldsymbol{v}, \boldsymbol{u}^{\prime}, \boldsymbol{v}^{\prime}, \boldsymbol{x} ; A\right)
$$

which implies that

$$
\mathbb{P}(\boldsymbol{v} \stackrel{A}{\rightarrow} \boldsymbol{x})=\mathbb{P}\left(E^{\prime}(\boldsymbol{v}, \boldsymbol{x} ; A)\right)+\sum_{\left(\boldsymbol{u}^{\prime}, \boldsymbol{v}^{\prime}\right)} \mathbb{P}\left(E\left(\boldsymbol{v}, \boldsymbol{u}^{\prime}, \boldsymbol{v}^{\prime}, \boldsymbol{x} ; A\right)\right)
$$

Defining

$$
E^{\prime \prime}\left(\boldsymbol{v}, \boldsymbol{u}^{\prime}, \boldsymbol{v}^{\prime} ; A\right)=\left\{E^{\prime}\left(\boldsymbol{v}, \boldsymbol{u}^{\prime} ; A\right) \text { occurs on } \widetilde{C}^{\left(\boldsymbol{u}^{\prime}, \boldsymbol{v}^{\prime}\right)}(\boldsymbol{v})\right\},
$$

the event $E\left(\boldsymbol{v}, \boldsymbol{u}^{\prime}, \boldsymbol{v}^{\prime}, \boldsymbol{x} ; A\right)$ can be rewritten as

$$
\begin{aligned}
E\left(\boldsymbol{v}, \boldsymbol{u}^{\prime}, \boldsymbol{v}^{\prime}, \boldsymbol{x} ; A\right)= & E^{\prime \prime}\left(\boldsymbol{v}, \boldsymbol{u}^{\prime}, \boldsymbol{v}^{\prime} ; A\right) \cap\left\{\left(\boldsymbol{u}^{\prime}, \boldsymbol{v}^{\prime}\right) \text { occupied }\right\} \\
& \cap\left\{\boldsymbol{v}^{\prime} \rightarrow \boldsymbol{x} \text { occurs in } \Lambda \backslash \widetilde{\boldsymbol{C}}^{\left(\boldsymbol{u}^{\prime}, \boldsymbol{v}^{\prime}\right)}(\boldsymbol{v})\right\} .
\end{aligned}
$$

Using Lemma 3.3, this gives

$$
\begin{aligned}
\mathbb{P}(\boldsymbol{v} \stackrel{A}{\rightarrow} \boldsymbol{x})= & \mathbb{P}\left(E^{\prime}(\boldsymbol{v}, \boldsymbol{x} ; A)\right) \\
& +p \sum_{\left(\boldsymbol{u}^{\prime}, \boldsymbol{v}^{\prime}\right)} D\left(\boldsymbol{v}^{\prime}-\boldsymbol{u}^{\prime}\right) \mathbb{E}\left(I\left[E^{\prime \prime}\left(\boldsymbol{v}, \boldsymbol{u}^{\prime}, \boldsymbol{v}^{\prime} ; A\right)\right] \tau^{\widetilde{C}^{\left(\boldsymbol{u}^{\prime}, \boldsymbol{v}^{\prime}\right)}(\boldsymbol{v})}\left(\boldsymbol{v}^{\prime}, \boldsymbol{x}\right)\right)
\end{aligned}
$$


We note that $E^{\prime \prime}\left(\boldsymbol{v}, \boldsymbol{u}^{\prime}, \boldsymbol{v}^{\prime} ; A\right)$ in (3.21) can be replaced by $E^{\prime}\left(\boldsymbol{v}, \boldsymbol{u}^{\prime} ; A\right)$, since $E^{\prime}\left(\boldsymbol{v}, \boldsymbol{u}^{\prime} ; A\right)$ is independent of the status of $\left(\boldsymbol{u}^{\prime}, \boldsymbol{v}^{\prime}\right)$, due to the orientation. Using this observation, and inserting the identity (3.13) into (3.21), we obtain

$$
\begin{aligned}
\mathbb{P}(\boldsymbol{v} \stackrel{A}{\rightarrow} \boldsymbol{x})= & \mathbb{P}\left(E^{\prime}(\boldsymbol{v}, \boldsymbol{x} ; A)\right)+p \sum_{\left(\boldsymbol{u}^{\prime}, \boldsymbol{v}^{\prime}\right)} D\left(\boldsymbol{v}^{\prime}-\boldsymbol{u}^{\prime}\right) \mathbb{P}\left(E^{\prime}\left(\boldsymbol{v}, \boldsymbol{u}^{\prime} ; A\right)\right) \tau\left(\boldsymbol{x}-\boldsymbol{v}^{\prime}\right) \\
& -p \sum_{\left(\boldsymbol{u}^{\prime}, \boldsymbol{v}^{\prime}\right)} D\left(\boldsymbol{v}^{\prime}-\boldsymbol{u}^{\prime}\right) \mathbb{E}_{1}\left(I\left[E^{\prime}\left(\boldsymbol{v}, \boldsymbol{u}^{\prime} ; A\right)\right] \mathbb{P}_{2}\left(\boldsymbol{v}^{\prime} \stackrel{\widetilde{C}_{1}^{\left(\boldsymbol{u}^{\prime}, v^{\prime}\right)}(\boldsymbol{v})}{\longrightarrow} \boldsymbol{x}\right)\right) .
\end{aligned}
$$

In the last term on the right side, we have introduced subscripts for $\widetilde{C}$ and the expectations, to indicate to which expectation $\widetilde{C}$ belongs.

Let

$$
\pi^{(1)}(\boldsymbol{x})=\sum_{(\boldsymbol{u}, \boldsymbol{v})} p D(\boldsymbol{v}-\boldsymbol{u}) \mathbb{E}_{0}\left(I[\mathbf{0} \Rightarrow \boldsymbol{u}] \mathbb{P}_{1}\left(E^{\prime}\left(\boldsymbol{v}, \boldsymbol{x} ; \widetilde{C}_{0}^{(\boldsymbol{u}, \boldsymbol{v})}(\mathbf{0})\right)\right)\right) .
$$

Inserting (3.22) into (3.14), and using (3.23), we have

$$
\begin{aligned}
\tau(\boldsymbol{x})= & \delta_{\mathbf{0}, \boldsymbol{x}}+\pi^{(0)}(\boldsymbol{x})-\pi^{(1)}(\boldsymbol{x})+\sum_{(\boldsymbol{u}, \boldsymbol{v})}\left(\delta_{\mathbf{0}, \boldsymbol{u}}+\pi^{(0)}(\boldsymbol{u})-\pi^{(1)}(\boldsymbol{u})\right) p D(\boldsymbol{v}-\boldsymbol{u}) \tau(\boldsymbol{x}-\boldsymbol{v}) \\
& +\sum_{(\boldsymbol{u}, \boldsymbol{v})} p D(\boldsymbol{v}-\boldsymbol{u}) \sum_{\left(\boldsymbol{u}^{\prime}, \boldsymbol{v}^{\prime}\right)} p D\left(\boldsymbol{v}^{\prime}-\boldsymbol{u}^{\prime}\right) \\
& \times \mathbb{E}_{0}\left(I[\mathbf{0} \Rightarrow \boldsymbol{u}] \mathbb{E}_{1}\left(I\left[E^{\prime}\left(\boldsymbol{v}, \boldsymbol{u}^{\prime} ; \tilde{C}_{0}^{(\boldsymbol{u}, \boldsymbol{v})}(\mathbf{0})\right)\right] \mathbb{P}_{2}\left(\boldsymbol{v}^{\prime} \stackrel{\widetilde{C}_{1}^{\left(u^{\prime}, \boldsymbol{v}^{\prime}\right)}(\boldsymbol{v})}{\longrightarrow} \boldsymbol{x}\right)\right)\right) .
\end{aligned}
$$

This completes the second stage of the expansion.

We now repeat this procedure recursively, rewriting $\mathbb{P}_{2}\left(\boldsymbol{v}^{\prime} \stackrel{\widetilde{C}_{1}^{\left(u^{\prime}, v^{\prime}\right)}(\boldsymbol{v})}{\longrightarrow} \boldsymbol{x}\right)$ using (3.22), and so on. This procedure stops after a finite number of steps, because each appearance of the last term of (3.22) uses up at least one unit of time, and the last term of (3.22) will vanish as soon as the temporal component of $\boldsymbol{v}^{\prime}$ exceeds that of $\boldsymbol{x}$. This leads to (3.2) and (3.3), with $\pi^{(0)}$ and $\pi^{(1)}$ given by (3.4) and (3.23), and, for $N \geqslant 2$,

$$
\begin{aligned}
\pi^{(N)}(\boldsymbol{x})= & \sum_{\left(\boldsymbol{u}_{0}, \boldsymbol{v}_{0}\right)} \ldots \sum_{\left(\boldsymbol{u}_{N-1}, \boldsymbol{v}_{N-1}\right)}\left[\prod_{i=0}^{N-1} p D\left(\boldsymbol{v}_{i}-\boldsymbol{u}_{i}\right)\right] \mathbb{E}_{0} I\left[\mathbf{0} \Rightarrow \boldsymbol{u}_{0}\right] \\
& \times \mathbb{E}_{1} I\left[E^{\prime}\left(\boldsymbol{v}_{0}, \boldsymbol{u}_{1} ; \widetilde{C}_{0}\right)\right] \cdots \mathbb{E}_{N-1} I\left[E^{\prime}\left(\boldsymbol{v}_{N-2}, \boldsymbol{u}_{N-1} ; \widetilde{C}_{N-2}\right)\right] \\
& \times \mathbb{E}_{N} I\left[E^{\prime}\left(\boldsymbol{v}_{N-1}, \boldsymbol{x} ; \widetilde{C}_{N-1}\right)\right] .
\end{aligned}
$$

Here, we have used the abbreviation $\widetilde{C}_{j}=\widetilde{C}_{j}^{\left(\boldsymbol{u}_{j}, \boldsymbol{v}_{j}\right)}\left(\boldsymbol{v}_{j-1}\right)$.

\subsection{The Nguyen-Yang expansion}

In this section we recall the derivation of the Nguyen-Yang expansion from [31]. As explained at the beginning of Section 3, we will use the Nguyen-Yang expansion only to prove the bound on $p \partial_{p} \hat{\pi}_{m}(k)$ of Proposition 2.2(iii). This proof will be given in Section 4.4. 
For $t=0,1,2, \ldots$, we define $W_{n, t}(x)$ to be the event that $(0,0) \rightarrow(x, n)$ with exactly $t$ occupied pivotal bonds for the connection, and let

$$
\tau_{n, t}(x)=\mathbb{P}_{p}\left(W_{n, t}(x)\right)
$$

By definition, $\tau_{n}(x)=\sum_{t=0}^{\infty} \tau_{n, t}(x)$. We will rewrite $\tau_{n, t}(x)$ in terms of a repulsive interaction between the sausages in the string of sausages representing the connection $(0,0) \rightarrow(x, n)$. When $W_{n, t}(x)$ occurs, there are exactly $t+1$ sausages.

Given a bond $b=\{(x, n),(y, n+1)\}$, let $\bar{b}=(y, n+1)$ be the "top" of $b$, and $\underline{b}=(x, n)$ the "bottom" of $b$. We will write $\bar{b}<\bar{b}$ ' to mean that the temporal component of $\bar{b}$ is less than that of $\bar{b}^{\prime}$, and, in an abuse of notation, we write $\bar{b}<n$ when the temporal component of $\bar{b}$ is less than $n$. For $t \geqslant 1$, let

$$
B_{t}(n)=\left\{\vec{b}=\left(b_{1}, \ldots, b_{t}\right): 0<\bar{b}_{1}<\cdots<\bar{b}_{t} \leqslant n\right\}
$$

denote the ordered vectors of $t$ bonds, up to time $n$. Given $\vec{b} \in B_{t}(n)$, we define $\bar{b}_{0}=(0,0), \underline{b}_{t+1}=(x, n)$. We also define

$$
T(\vec{b},(x, n))=\bigcap_{i=1}^{t}\left\{b_{i} \text { occupied }\right\} \bigcap_{i=0}^{t}\left\{\bar{b}_{i} \Rightarrow \underline{b}_{i+1}\right\} .
$$

Note that if $T(\vec{b},(x, n))$ occurs, then the only possible candidates for occupied pivotal bonds for the event $(0,0) \rightarrow(x, n)$ are the elements of $\vec{b}$. We define the random variables

$$
K[i, j]=\prod_{i \leqslant s<t \leqslant j}\left(1+U_{s t}\right) \quad \text { with } \quad U_{i j}=-I\left[\bar{b}_{i} \Rightarrow \underline{b}_{j+1}\right] .
$$

The product in (3.29) is 0 or 1 . If $K[0, t]=1$ and $T(\vec{b},(x, n))$ occurs, then the occupied pivotal bonds for the event $(0,0) \rightarrow(x, n)$ are precisely the elements of $\vec{b}$. Therefore

$$
\tau_{n, t}(x)=\sum_{\vec{b} \in B_{t}(n)} \mathbb{E}_{p}[I[T(\vec{b},(x, n))] K[0, t]] .
$$

The lace expansion involves a decomposition of $K[0, t]$. To describe this, we need some standard terminology [8,29]. A graph on an interval $[a, b]$ is a set $\Gamma=$ $\left\{i_{1} j_{1}, \ldots, i_{M} j_{M}\right\}$ of edges, with $a \leqslant i_{l}<j_{l} \leqslant b$ for each $l$, and with $M \geqslant 0$. We say that a graph $\Gamma$ is connected on $[a, b]$ if $\bigcup_{i j \in \Gamma}[i, j]=[a, b]$. We denote the set of connected graphs on $[a, b]$ by $\mathcal{G}[a, b]$, and let

$$
J[0, s]=\sum_{\Gamma \in \mathcal{G}[0, s]} \prod_{i j \in \Gamma} U_{i j} .
$$

Expanding the product in (3.29) leads to a sum over all graphs, and by partitioning according to the support of the connected component of 0 , the following decomposition 
emerges (see $[29,(5.5 .9)]$ for more details)

$$
K[0, t+1]=K[1, t+1]+\sum_{s=1}^{t} J[0, s] K[s+1, t+1]+J[0, t+1] \quad(t \geqslant 0) .
$$

In the right side of the above equation, the middle term is taken to be 0 if $t \leqslant 1$, and $K[a, a]=1$. Let

$$
\begin{aligned}
& \pi_{m, 0}(x)=\mathbb{P}_{p}((0,0) \Rightarrow(x, m))-\delta_{0, x} \delta_{0, m}=\tau_{m, 0}(x)-\delta_{0, x} \delta_{0, m}, \\
& \pi_{m, s}(x)=\sum_{\vec{b} \in B_{s}(m)} \mathbb{E}_{p}[I[T(\vec{b},(x, m))] J[0, s]] \quad(s \geqslant 1) .
\end{aligned}
$$

It can be seen from the above definitions that $\pi_{m, s}(x)=0$ whenever $m=0,1$ or $s>m$. Substitution of (3.32) into (3.30), followed by application of the Markov property, then gives the recursion formula

$$
\tau_{n+1, t+1}(x)=p\left(D * \tau_{n, t}\right)(x)+\sum_{m=2}^{n} \sum_{s=0}^{t} p\left(\pi_{m, s} * D * \tau_{n-m, t-s}\right)(x)+\pi_{n+1, t+1}(x) \quad(t \geqslant 0) .
$$

Summing (3.35) over $t$ then gives the basic recursion formula (3.1), with

$$
\pi_{m}(x)=\sum_{s=0}^{m} \pi_{m, s}(x) .
$$

Finally, we rewrite $\pi_{m, s}(x)$ in terms of laces. A lace on $[a, b]$ is an element of $\mathcal{G}[a, b]$ such that the removal of any edge will result in a disconnected graph. Given a connected graph $\Gamma \in \mathcal{G}[a, b]$, we define the lace $\mathrm{L}_{\Gamma} \subset \Gamma$ to be the graph consisting of edges $s_{1} t_{1}, s_{2} t_{2}, \ldots$ given by

$$
\begin{aligned}
& t_{1}=\max \{t: a t \in \Gamma\}, \quad s_{1}=a, \\
& t_{i+1}=\max \left\{t: \exists s \leqslant t_{i} \text { such that } s t \in \Gamma\right\}, \quad s_{i+1}=\min \left\{s: s t_{i+1} \in \Gamma\right\} .
\end{aligned}
$$

(It is not hard to check that $L_{\Gamma}$ is indeed a lace.) Given a lace $L$, let $\mathcal{C}(L)$ denote the set of compatible edges, i.e., the set of edges $i j$ such that $L_{L \cup\{i j\}}=L$. Define $\mathcal{L}^{(N)}[a, b]$ to be the set of laces on the interval $[a, b]$, consisting of exactly $N$ edges. It is then a standard calculation $[8,29]$ to conclude that

$$
\pi_{m, s}(x)=\sum_{N=0}^{\infty}(-1)^{N} \Pi_{m, s}^{(N)}(x),
$$

with $\Pi_{m, s}^{(0)}(x)=\pi_{m, 0}(x) \delta_{0, s}$ and

$$
\begin{aligned}
\Pi_{m, s}^{(N)}(x)= & (-1)^{N} \sum_{\vec{b} \in B_{s}(m)} \mathbb{E}_{p}[I[T(\vec{b},(x, m))] \\
& \left.\times \sum_{L \in \mathcal{L}^{(N)}[0, s]} \prod_{i j \in L} U_{i j} \prod_{i^{\prime} j^{\prime} \in \mathcal{C}(L)}\left(1+U_{i^{\prime} j^{\prime}}\right)\right] \quad(N \geqslant 1) .
\end{aligned}
$$


The combination $(-1)^{N} \prod_{i j \in L} U_{i j}$ is either 0 or 1 , so $\Pi_{m, s}^{(N)}(x) \geqslant 0$. The above gives the identity (3.2), but now with the decomposition

$$
\pi_{m}(x)=\sum_{N=0}^{\infty}(-1)^{N} \Pi_{m}^{(N)}(x) \quad \text { with } \quad \Pi_{m}^{(N)}(x)=\sum_{s=0}^{\infty} \Pi_{m, s}^{(N)}(x)
$$

in place of (3.2).

\section{Proof of Proposition 2.2}

In this section, we prove Proposition 2.2. For Proposition 2.2(i)-(ii), we will use the Hara-Slade expansion of Section 3.1, whereas for Proposition 2.2(iii) we will use the Nguyen-Yang expansion of Section 3.2. We begin in Section 4.1 by indicating how $\pi^{(N)}(\boldsymbol{x})$ of (3.25) can be bounded in terms of the two-point function, with these bounds conveniently organised using diagrams. The diagrams are then estimated in Section 4.2. This will be used in Section 4.3 to prove the bounds on $\pi(\boldsymbol{x})$ of Proposition 2.2(i)-(ii). Finally, in Section 4.4, the diagrammatic estimates will be extended to the Nguyen-Yang expansion, to estimate $p \partial_{p} \pi(\boldsymbol{x})$ and prove Proposition 2.2(iii).

\subsection{Diagrammatic bounds}

\subsubsection{Diagrams}

We now indicate how useful diagrammatic bounds can be obtained for $\pi^{(N)}(\boldsymbol{x})$, using the method of [20]. A key ingredient in the diagrammatic estimates is the following special case of the BK inequality (see [18]). Let $V_{1}, \ldots, V_{n}$ be sets of oriented lattice paths, and let $E_{i}$ be the event that at least one of the paths in $V_{i}$ is occupied. The event $E_{1} \circ \cdots \circ E_{n}$ represents the event that there exist pairwise bond-disjoint occupied paths $\omega_{i} \in V_{i}(i=1,2, \ldots, n)$. Then the inequality

$$
\mathbb{P}\left(E_{1} \circ \cdots \circ E_{n}\right) \leqslant \mathbb{P}\left(E_{1}\right) \mathbb{P}\left(E_{2}\right) \ldots \mathbb{P}\left(E_{n}\right)
$$

follows from the BK inequality and the fact that the $E_{i}$ are increasing events.

Using (4.1), we immediately obtain the estimate

$$
\pi^{(0)}(\boldsymbol{x})=\mathbb{P}(\mathbf{0} \Rightarrow \boldsymbol{x})-\delta_{\boldsymbol{0}, \boldsymbol{x}}=\mathbb{P}((\mathbf{0} \rightarrow \boldsymbol{x}) \circ(\mathbf{0} \rightarrow \boldsymbol{x}))-\delta_{\mathbf{0}, \boldsymbol{x}} \leqslant \tau(\boldsymbol{x})^{2}-\delta_{\mathbf{0}, \boldsymbol{x}} .
$$

To bound $\pi^{(N)}$ for $N \geqslant 1$, we estimate the nested expectation (3.25) from the inside out (right to left). For the innermost expectation $\mathbb{E}_{N}$, we first observe that $E^{\prime}\left(\boldsymbol{v}_{N-1}, \boldsymbol{x} ; \widetilde{C}_{N-1}\right)$ is a subset of the event that there exist $\boldsymbol{w}_{N} \in \widetilde{C}_{N-1}$ and $\boldsymbol{t} \in \Lambda$ with four disjoint paths realising the connections $\boldsymbol{v}_{N-1} \rightarrow \boldsymbol{t}, \boldsymbol{t} \rightarrow \boldsymbol{w}_{N}, \boldsymbol{w}_{N} \rightarrow \boldsymbol{x}, \boldsymbol{t} \rightarrow \boldsymbol{x}$. Applying the BK inequality gives

$$
\begin{aligned}
& \mathbb{E}_{N} I\left[E^{\prime}\left(\boldsymbol{v}_{N-1}, \boldsymbol{x} ; \widetilde{C}_{N-1}\right)\right] \\
& \quad \leqslant \sum_{\boldsymbol{t}, \boldsymbol{w}_{N} \in \Lambda} I\left[\boldsymbol{w}_{N} \in \widetilde{C}_{N-1}\right] \tau\left(\boldsymbol{t}-\boldsymbol{v}_{N-1}\right) \tau\left(\boldsymbol{w}_{N}-\boldsymbol{t}\right) \tau\left(\boldsymbol{x}-\boldsymbol{w}_{N}\right) \tau(\boldsymbol{x}-\boldsymbol{t}) .
\end{aligned}
$$


The indicator $I\left[\boldsymbol{w}_{N} \in \widetilde{C}_{N-1}\right]$ is a random variable for the expectation $\mathbb{E}_{N-1}$ that must be treated in conjunction with the event $E^{\prime}\left(\boldsymbol{v}_{N-2}, \boldsymbol{u}_{N-1} ; \widetilde{C}_{N-2}\right)$ (when $N \geqslant 2$ ). It is not difficult to show (see [20, Lemma 2.5] or [29, Lemma 5.5.8] for details) that

$$
\begin{aligned}
& \mathbb{E}_{i}\left(I\left[E^{\prime}\left(\boldsymbol{v}_{i-1}, \boldsymbol{u}_{i} ; \widetilde{C}_{i-1}\right)\right] I\left[\boldsymbol{w}_{i+1} \in \widetilde{C}_{i}\right]\right) \\
& \leqslant \sum_{\boldsymbol{w}_{i}, z_{i} t, \boldsymbol{y}} I\left[\boldsymbol{w}_{i} \in \widetilde{C}_{i-1}\right] \tau\left(\boldsymbol{w}_{i+1}-\boldsymbol{z}_{i}\right) \tau\left(\boldsymbol{u}_{i}-\boldsymbol{w}_{i}\right) \tau\left(\boldsymbol{w}_{i}-t\right) \\
& \quad \times\left(\tau\left(\boldsymbol{t}-\boldsymbol{v}_{i-1}\right) \tau\left(\boldsymbol{z}_{i}-\boldsymbol{t}\right) \tau\left(\boldsymbol{u}_{i}-\boldsymbol{z}_{i}\right)\right. \\
& \left.\quad+\tau\left(\boldsymbol{z}_{i}-\boldsymbol{v}_{i-1}\right) \tau\left(\boldsymbol{t}-\boldsymbol{z}_{i}\right) \tau\left(\boldsymbol{u}_{i}-\boldsymbol{t}\right)\right) .
\end{aligned}
$$

Finally, the expectation $\mathbb{E}_{0}$ is estimated using

$$
\mathbb{E}_{0}\left(I\left[\mathbf{0} \Rightarrow \boldsymbol{u}_{0}\right] I\left[\boldsymbol{w}_{1} \in \widetilde{C}_{0}\right]\right) \leqslant \sum_{z_{0}} \tau\left(\boldsymbol{u}_{0}\right) \tau\left(z_{0}\right) \tau\left(\boldsymbol{u}_{0}-z_{0}\right) \tau\left(\boldsymbol{w}_{1}-z_{0}\right),
$$

which follows from the BK inequality and the fact that the intersection of the events on the left side is a subset of the event that there exists $z_{0}$ with four disjoint connections $\mathbf{0} \rightarrow \boldsymbol{u}_{0}, \mathbf{0} \rightarrow z_{0}, z_{0} \rightarrow \boldsymbol{u}_{0}, z_{0} \rightarrow \boldsymbol{w}_{1}$.

The upper bounds (4.3)-(4.5) have a convenient diagrammatic representation, as illustrated in Fig. 6. Diagrams are interpreted as follows. Each diagram vertex is assigned a lattice variable and each diagram line is assigned a two-point function evaluated at the (oriented) difference of its endpoint variables. Unlabelled vertices are summed over all possible values. In Fig. 7, and in later figures, we will also use a pair of short line segments to indicate vertices that are separated by unit time; these correspond in Fig. 7 to the pivotal bonds $\left(\boldsymbol{u}_{i}, \boldsymbol{v}_{i}\right)$ of (3.25) and carry weight $\tau\left(\boldsymbol{v}_{i}-\boldsymbol{u}_{i}\right)=p D\left(\boldsymbol{v}_{i}-\boldsymbol{u}_{i}\right)$. More generally, a diagram is a graph with multiple edges between vertices allowed, with factors of $\tau$ associated to each edge and summed as described above.

In Fig. 6, we also show the effect of shifting the diagram line $\tau\left(\boldsymbol{w}_{i+1}-\boldsymbol{z}_{i}\right)$ to the diagram arising in the upper bound for the expectation $\mathbb{E}_{i+1}$, rather than keeping it in the upper bound for $\mathbb{E}_{i}$, for $i=0, \ldots, N-1$. With this shift, a convenient recursive structure emerges. To see this, we define

$$
\begin{aligned}
P_{1}\left(\boldsymbol{u}_{0}, z_{0}\right)= & \tau\left(\boldsymbol{u}_{0}\right) \tau\left(z_{0}\right) \tau\left(\boldsymbol{u}_{0}-\boldsymbol{z}_{0}\right), \\
P_{2}\left(\boldsymbol{u}_{i-1}, z_{i-1}, \boldsymbol{u}_{i}, \boldsymbol{z}_{i}\right)= & \sum_{\boldsymbol{t}, \boldsymbol{w}, \boldsymbol{v}_{i-1}} p D\left(\boldsymbol{v}_{i-1}-\boldsymbol{u}_{i-1}\right) \tau(\boldsymbol{w}-\boldsymbol{t}) \tau\left(\boldsymbol{u}_{i}-\boldsymbol{w}\right) \tau\left(\boldsymbol{w}-\boldsymbol{z}_{i-1}\right) \\
& \times\left(\tau\left(\boldsymbol{z}_{i}-\boldsymbol{v}_{i-1}\right) \tau\left(\boldsymbol{t}-\boldsymbol{z}_{i}\right) \tau\left(\boldsymbol{u}_{i}-\boldsymbol{t}\right)\right. \\
& \left.+\tau\left(\boldsymbol{t}-\boldsymbol{v}_{i-1}\right) \tau\left(\boldsymbol{z}_{i}-\boldsymbol{t}\right) \tau\left(\boldsymbol{u}_{i}-\boldsymbol{z}_{i}\right)\right), \\
P_{3}\left(\boldsymbol{u}_{N-1}, \boldsymbol{z}_{N-1}, \boldsymbol{x}\right)= & \sum_{\boldsymbol{t}, \boldsymbol{w}, \boldsymbol{v}_{N-1}} p D\left(\boldsymbol{v}_{N-1}-\boldsymbol{u}_{N-1}\right) \tau\left(\boldsymbol{t}-\boldsymbol{v}_{N-1}\right) \tau(\boldsymbol{x}-\boldsymbol{t}) \tau(\boldsymbol{x}-\boldsymbol{w}) \\
& \times \tau(\boldsymbol{w}-\boldsymbol{t}) \tau\left(\boldsymbol{w}-\boldsymbol{z}_{N-1}\right) .
\end{aligned}
$$

The right sides of the above equations correspond to the diagrams appearing in Fig. 6 after shifting lines, with the difference that the bonds $\left(\boldsymbol{u}_{i-1}, \boldsymbol{v}_{i-1}\right)$ appear in (4.7)-(4.8) but not in Fig. 6. For $N \geqslant 1$, we define 
R. VAN DER HOFSTAD, G. SLADE / Ann. I. H. Poincaré - PR 39 (2003) 413-485

$(4.3)$

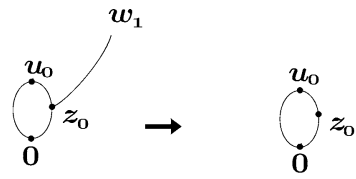

$(4.4)$
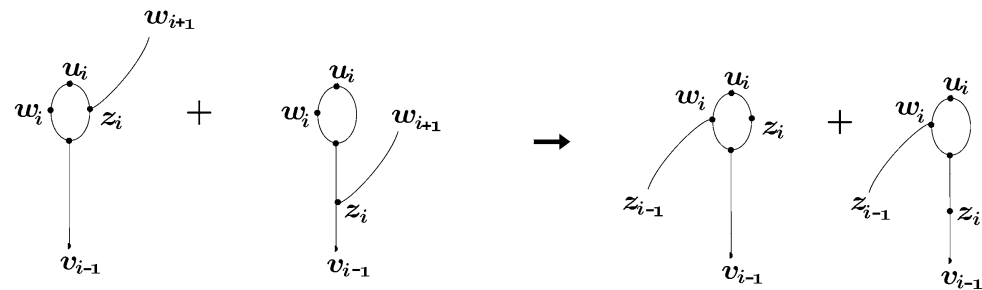

$(4.5)$

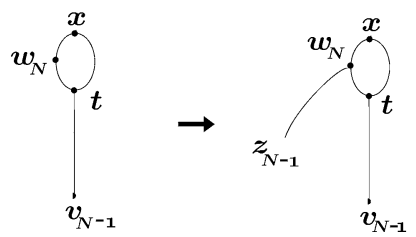

Fig. 6. Diagrams representing (4.3)-(4.5), showing also the result of a shifted line.
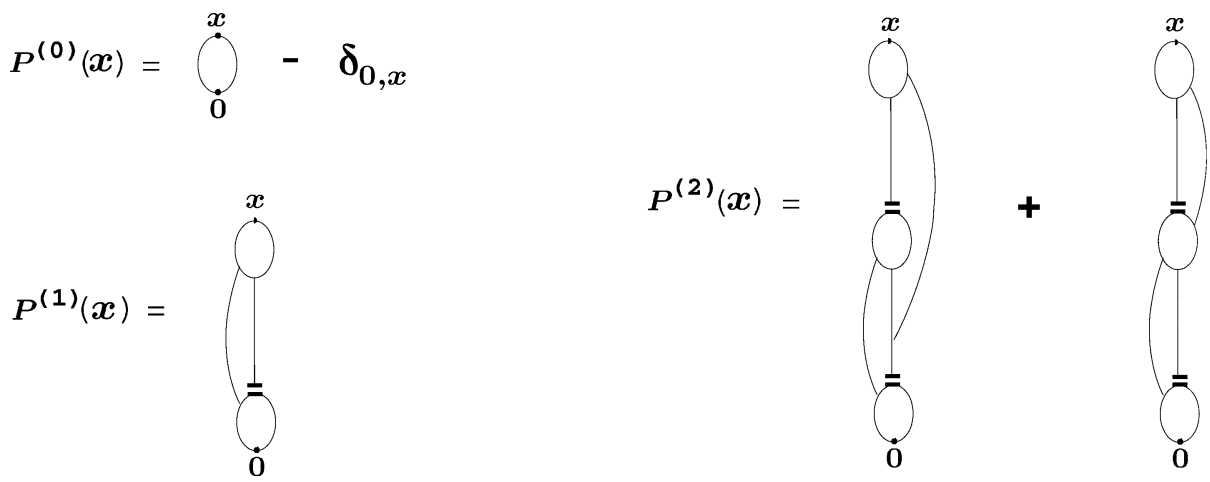

Fig. 7. Diagrams representing $P^{(N)}(\boldsymbol{x}), N=0,1,2$.

$$
\begin{aligned}
P^{(N)}(\boldsymbol{x})= & \sum_{\left(\boldsymbol{u}_{0}, z_{0}\right)} \ldots \sum_{\left(\boldsymbol{u}_{N-1}, z_{N-1}\right)} P_{1}\left(\boldsymbol{u}_{0}, \boldsymbol{z}_{0}\right)\left[\prod_{i=1}^{N-1} P_{2}\left(\boldsymbol{u}_{i-1}, \boldsymbol{z}_{i-1}, \boldsymbol{u}_{i}, \boldsymbol{z}_{i}\right)\right] \\
& \times P_{3}\left(\boldsymbol{u}_{N-1}, \boldsymbol{z}_{N-1}, \boldsymbol{x}\right),
\end{aligned}
$$

where the empty product arising when $N=1$ is defined to be 1 . We also define the bubble diagram $B(\boldsymbol{x})=\tau(\boldsymbol{x})^{2}$, and set $P^{(0)}(\boldsymbol{x})=B(\boldsymbol{x})-\delta_{\boldsymbol{0}, \boldsymbol{x}}$. The inequalities (4.3)(4.5) can then be combined with (3.25) to give

$$
\pi^{(N)}(\boldsymbol{x}) \leqslant P^{(N)}(\boldsymbol{x}) \quad(N \geqslant 0) .
$$


The diagrams $P^{(N)}(\boldsymbol{x})$ are depicted in Fig. 7, for $N=0,1,2$.

The inequality (4.10) shows that $\pi^{(N)}(\boldsymbol{x})$ can be bounded by a sum of products of two-point functions $\tau(\boldsymbol{y})$, with the temporal component of $\boldsymbol{y}$ no larger than that of $\boldsymbol{x}$. These inequalities will provide the key ingredient needed to prove Proposition 2.2, which simply asserts that certain bounds on $\tau(\boldsymbol{y})$ imply bounds on $\pi(\boldsymbol{x})$.

\subsubsection{Diagrammatic constructions}

We now introduce some diagrammatic constructions that will be useful to estimate $P^{(N)}(\boldsymbol{x})$. A related procedure was employed in [22].

DEFINITION 4.1. - (a) Given a diagram, and given any line $\lambda$ of the diagram, construction $1^{\lambda}(\boldsymbol{y})$ is the operation in which a new vertex $\boldsymbol{y}$ is inserted in line $\lambda$. Explicitly, this means that the two-point function $\tau(\boldsymbol{v}-\boldsymbol{u})$ (say) corresponding to line $\lambda$ is replaced by $\tau(\boldsymbol{v}-\boldsymbol{y}) \tau(\boldsymbol{y}-\boldsymbol{u})$.

(b) Construction $1^{\lambda}(l)$ is the operation in which construction $1^{\lambda}(y, l)$ is performed and then followed by summation over $y$ (leaving l fixed). Explicitly, this means that $\tau_{j-i}(v-u)$ corresponding to line $\lambda$ is replaced by $\sum_{y} \tau_{j-l}(v-y) \tau_{l-i}(y-u)$.

In the diagram $P^{(0)}$, we declare one of the lines in the bubble to be admissible. For $N \geqslant 1$, we declare the lines $\tau\left(\boldsymbol{t}-\boldsymbol{v}_{N-1}\right)$ and $\tau(\boldsymbol{x}-\boldsymbol{t})$ appearing in (4.8) to be admissible. These are the lines on $P_{3}$ from which the terms of $P_{2}$ can be obtained by adding an additional vertex. Writing $P^{(N-1)}\left(x ; 1^{\lambda}(z)\right)$ for the result of applying construction $1^{\lambda}(z)$ to $P^{(N-1)}(x)$, it then follows from (4.9) that

$$
P^{(N)}(\boldsymbol{x})=\sum_{\boldsymbol{u}, \boldsymbol{z}} \sum_{\lambda} P^{(N-1)}\left(\boldsymbol{u} ; 1^{\lambda}(z)\right) P_{3}(\boldsymbol{u}, \boldsymbol{z}, \boldsymbol{x}) \quad(N \geqslant 1),
$$

where the sum over $\lambda$ is over two admissible lines for $N \geqslant 2$ and over one admissible line for $N=1$.

We will formalise the operation occuring in (4.11) in a definition. For this, we define

$$
\begin{aligned}
& L^{(0)}(\boldsymbol{w}, \boldsymbol{t}, \boldsymbol{x})=\tau(\boldsymbol{x}-\boldsymbol{w}) \tau(\boldsymbol{x}-\boldsymbol{t}), \\
& L^{(1)}(\boldsymbol{u}, \boldsymbol{z}, \boldsymbol{x})=\tau(\boldsymbol{x}-\boldsymbol{z}) \sum_{\boldsymbol{v}} p D(\boldsymbol{v}-\boldsymbol{u}) \tau(\boldsymbol{x}-\boldsymbol{v}) .
\end{aligned}
$$

Given a diagram $F(\boldsymbol{u})$ with two labelled vertices having labels $\mathbf{0}$ and $\boldsymbol{u}$, and with a certain set of admissible lines indexed by $\lambda$, let $F\left(\boldsymbol{u} ; 1^{\lambda}(z)\right)$ denote the result of applying construction $1^{\lambda}(z)$. It then follows from (4.8) that

$$
P_{3}(\boldsymbol{u}, \boldsymbol{z}, \boldsymbol{x})=\sum_{\boldsymbol{t}, \boldsymbol{w}} L^{(1)}\left(\boldsymbol{u}, \boldsymbol{z}, \boldsymbol{w} ; 1^{\alpha}(\boldsymbol{t})\right) L^{(0)}(\boldsymbol{t}, \boldsymbol{w}, \boldsymbol{x}),
$$

where $\alpha$ is the line from $\boldsymbol{v}$ to $\boldsymbol{w}$ occuring in $L^{(1)}(\boldsymbol{u}, z, \boldsymbol{w})$ (with $\boldsymbol{v}$ the summation index in (4.13)).

DEFINITION 4.2. - Given a diagram $F(\boldsymbol{u})$ with two labelled vertices having labels $\mathbf{0}$ and $\boldsymbol{u}$, and with a certain set of admissible lines indexed by $\lambda$, construction $2_{\boldsymbol{u}}^{(i)}(\boldsymbol{w})$ produces the diagram

$$
\widetilde{F}^{(i)}(\boldsymbol{w})=\sum_{\lambda} \sum_{\boldsymbol{u}, \boldsymbol{z}} F\left(\boldsymbol{u} ; 1^{\lambda}(\boldsymbol{z})\right) L^{(i)}(\boldsymbol{u}, \boldsymbol{z}, \boldsymbol{w}),
$$


and construction $E_{\boldsymbol{u}}(\boldsymbol{w})$ produces the diagram

$$
\widetilde{F}^{(i)}(\boldsymbol{w})=\sum_{\lambda} \sum_{\boldsymbol{u}, \boldsymbol{z}} F\left(\boldsymbol{u} ; 1^{\lambda}(\boldsymbol{z})\right) P_{3}(\boldsymbol{u}, \boldsymbol{z}, \boldsymbol{w}),
$$

where the sum over $\lambda$ is the sum over admissible lines.

We may then understand the factor $P_{3}(\boldsymbol{u}, \boldsymbol{z}, \boldsymbol{x})$ in (4.11) as corresponding to application of construction $E_{\boldsymbol{u}}(\boldsymbol{x})$ to $P^{(N-1)}(\boldsymbol{u})$. By definition and (4.14), construction $E_{\boldsymbol{u}}(\boldsymbol{x})$ is equivalent to an application of construction $2_{\boldsymbol{u}}^{(1)}(\boldsymbol{w})$ followed by construction $2_{\boldsymbol{w}}^{(0)}(\boldsymbol{x})$, where the unique admissible line prior to applying construction $2_{w}^{(0)}(\boldsymbol{x})$ is the line from $\boldsymbol{v}$ to $\boldsymbol{w}$ added to the diagram in the application of construction $2_{\boldsymbol{u}}^{(1)}(\boldsymbol{w})$. (Here, $\boldsymbol{v}$ corresponds to the summation index in (4.13).) Thus $P^{(N)}(\boldsymbol{x})$ is obtained by $N$ applications of construction $E$ to the bubble diagram, for all $N \geqslant 1$. To estimate $P^{(N)}(\boldsymbol{x})$, it therefore suffices to estimate the bubble and to investigate the result of applying construction $E_{\boldsymbol{u}}(\boldsymbol{x})$ to a diagram whose behaviour is already understood. For this, it suffices to understand the result of applying construction $2_{u}^{(i)}(\boldsymbol{w})$ to a diagram whose behaviour is already understood.

\subsection{Estimation of diagrams}

The main goal of this section is to obtain an estimate for the diagrams $P^{(N)}$ of Section 4.1. The result, which will be at the heart of the proof of Proposition 2.2, is stated in Lemma 4.5. A secondary goal is to develop a general method for estimating diagrams, that will be useful also in Section 7.

We begin with a lemma that converts the Fourier-space bounds of (2.15) for $1 \leqslant m \leqslant n$ into $x$-space bounds for $0 \leqslant m \leqslant n+1$. The inclusion of $m=n+1$ in the $x$-space bounds is essential for obtaining the bounds of Proposition 2.2 for $0 \leqslant m \leqslant n+1$. It will be convenient in what follows to return to the $(x, m)$ notation for sites.

LEMMA 4.3. - Suppose that (2.15) holds for $1 \leqslant m \leqslant n$ and for some $p \in I$. Then there is a $K$, depending on $K_{f}$, such that for $0 \leqslant m \leqslant n+1$ and for that $p$, the following bounds hold:

$$
\begin{aligned}
\hat{\tau}_{m}(0) \leqslant K, \quad & \sum_{x}|x|^{2} \tau_{m}(x) \leqslant K \sigma^{2} m, \quad\left\|\tau_{m}\right\|_{\infty} \leqslant \begin{cases}\frac{K \beta}{m^{d / 2}} & (m \neq 0), \\
1 & (m=0),\end{cases} \\
& \sup _{x}|x|^{2} \tau_{m}(x) \leqslant K \sigma^{2} \beta\left(\frac{1}{m+1}\right)^{(d-2) / 2} .
\end{aligned}
$$

We will interpret the bound on $\left\|\tau_{m}\right\|_{\infty}$ of (4.17) as

$$
\left\|\tau_{m}\right\|_{\infty} \leqslant \begin{cases}\frac{K \beta}{(m+1)^{d / 2}} & (\text { if } m \neq 0 \text { is guaranteed }) \\ \frac{K}{(m+1)^{d / 2}} & \text { (otherwise) }\end{cases}
$$

Proof. - The bounds are all trivial for $m=0$. For $m \leqslant n$, the first two bounds are immediate from (2.15). For $m=n+1$, given $l \geqslant 0$ and $0 \leqslant j \leqslant l$ we use Boole's 
inequality and the Markov property to obtain

$$
\tau_{l}(x)=\mathbb{P}_{p}\left(\bigcup_{y}\{(0,0) \rightarrow(y, j) \rightarrow(x, l)\}\right) \leqslant \sum_{y} \tau_{j}(y) \tau_{l-j}(x-y) .
$$

Taking $l=n+1$ and $j=1$ in (4.20), we obtain $\hat{\tau}_{n+1}(0) \leqslant \hat{\tau}_{1}(0) \hat{\tau}_{n}(0)=p \hat{\tau}_{n}(0)$, which gives the first bound of (4.17) for $m=n+1$. The second bound can be obtained similarly, for $m=n+1$, using also the estimate $|x|^{2} \leqslant 2\left(|y|^{2}+|x-y|^{2}\right)$ and the definition of $\sigma$ in (1.3).

Next, we consider the third bound of (4.17). For $m=1$, we have $\left\|\tau_{1}\right\|_{\infty}=p\|D\|_{\infty} \leqslant$ $K \beta$, by the assumption above (1.2). For $m \geqslant 2$, we use (4.20) twice, as well as the inequality $\|h\|_{\infty} \leqslant\|\hat{h}\|_{1}$, to conclude that

$$
\tau_{m}(x) \leqslant\left(\tau_{1} * \tau_{1} * \tau_{m-2}\right)(x) \leqslant p^{2}\left\|\widehat{D}^{2} \hat{\tau}_{m-2}\right\|_{1} .
$$

Thus (2.15) implies (4.17) for all $m \leqslant n+2$.

For (4.18) with $m=1$, we use the first assumption of (1.2). For (4.18) with $m \geqslant 2$, we use (4.20) with $l=m$ and $j=\lfloor m / 2\rfloor$ to obtain

$$
\begin{aligned}
|x|^{2} \tau_{m}(x) & \leqslant 2 \sum_{y}\left(|y|^{2}+|x-y|^{2}\right) \tau_{\lfloor m / 2\rfloor}(y) \tau_{m-\lfloor m / 2\rfloor}(x-y) \\
& \leqslant 2\left(\left\|\tau_{m-\lfloor m / 2\rfloor}\right\|_{\infty} \sum_{y}|y|^{2} \tau_{\lfloor m / 2\rfloor}(y)+\left\|\tau_{\lfloor m / 2\rfloor}\right\|_{\infty} \sum_{y}|y|^{2} \tau_{m-\lfloor m / 2\rfloor}(y)\right) .
\end{aligned}
$$

Then (4.18) follows from (4.17) for $m \leqslant n+1$ (in fact for $m$ of order $2 n$ ).

As was explained above the statement of Proposition 2.2, (2.15) will follow once we establish Assumption 2.1 by proving Proposition 2.2. Therefore the bounds (4.17)(4.18) of Lemma 4.3 will also follow, for all $m \geqslant 0$.

Next, we give a simple example of an estimate for a diagram. Following the example, we will develop a more systematic approach to such estimates.

Example 4.4. - We define a diagram $G_{m}(x)$ by

$$
G_{m}(x)=\sum_{0 \leqslant j \leqslant k<m} \sum_{u, v, w} \tau_{j}(u) \tau_{k}(v) \tau_{k-j}(v-u) \tau_{1}(w-v) \tau_{m-k-1}(x-w) \tau_{m-j}(x-u) .
$$

This diagram is the contribution to $\pi_{m}^{(1)}(x)$ that arises when the topmost loop in the diagram $P_{m}^{(1)}(x)$ shrinks to the single vertex $(x, m)$. Note that all subscripts on the right side of (4.23) are at most $m$. To estimate $G_{m}=\sum_{x} G_{m}(x)$, we bound the second and sixth factors above by their maximum possible values to obtain

$$
G_{m} \leqslant \sum_{0 \leqslant j \leqslant k<m}\left\|\tau_{j}\right\|_{1}\left\|\tau_{k}\right\|_{\infty}\left\|\tau_{k-j}\right\|_{1}\left\|\tau_{1}\right\|_{1}\left\|\tau_{m-k-1}\right\|_{1}\left\|\tau_{m-j}\right\|_{\infty} .
$$


Assuming the bounds (4.17)-(4.19) for $\tau_{l}$ with $l \leqslant m$ gives

$$
G_{m} \leqslant K^{6} \beta \sum_{0 \leqslant j \leqslant k<m}(k+1)^{-d / 2}(m-j+1)^{-d / 2} \leqslant c K^{6} \beta(m+1)^{-d / 2} .
$$

In the above estimate, we used the fact that since $m-j>0$ we obtain a factor $\beta$ when using (4.19) to estimate $\left\|\tau_{m-j}\right\|_{\infty}$. In addition, we used the fact that for $d>4$ there is a constant $c=c(d)$ such that

$$
\sum_{0 \leqslant i \leqslant j \leqslant n}(j+1)^{-d / 2}(n-i+1)^{-d / 2} \leqslant c(n+1)^{-d / 2}
$$

holds uniformly in $n \geqslant 0$. To prove (4.26), we divide the sum into two cases, depending on whether $j \leqslant n-i$ or not. The contributions due to each case are equal, due to the symmetry present in the sum on the left side of (4.26). When $j \leqslant n-i$, we have $n-i \geqslant n / 2$, because $i \leqslant j$. Therefore, the left side of (4.26) is bounded above, as required, by

$$
\begin{aligned}
2 \cdot 2^{d / 2}(n+1)^{-d / 2} \sum_{0 \leqslant i \leqslant j \leqslant n}(j+1)^{-d / 2} & \leqslant 2 \cdot 2^{d / 2}(n+1)^{-d / 2} \sum_{j=0}^{\infty}(j+1)^{-(d-2) / 2} \\
& \leqslant c(n+1)^{-d / 2}
\end{aligned}
$$

Convergence of the above sums gives a sharp criterion requiring that $d$ be above the critical dimension 4.

Consider, in addition, the effect of associating to $G_{m}(x)$ a factor $|x|^{2}$ or $|x|^{4}$ before performing the sum over $x$. Using $|x|^{2} \leqslant 2\left(|u|^{2}+|x-u|^{2}\right.$ ) and (if we are considering $\left.|x|^{4}\right)|x|^{2} \leqslant 2\left(|v|^{2}+|x-v|\right)^{2}$, we can associate factors of $|x|^{2}$ to one or two distinct (when considering $|x|^{4}$ ) diagram lines. By (4.17) and (4.18), the effect of a factor $|x|^{2}$ on an upper bound on an $l^{1}$ or $l^{\infty}$ norm is to increase the estimate by a factor $\sigma^{2}$ times the temporal displacement of the diagram line. The latter is bounded by $m+1$, and we can thus conclude from (4.25) that

$$
\sum_{x}|x|^{q} G_{m}(x) \leqslant C K^{6} \beta \sigma^{q}(m+1)^{(q-d) / 2} \quad(q \in\{0,2,4\}) .
$$

This completes Example 4.4.

We now come to the principal lemma of this section. In view of (4.10) and the fact that $\pi_{m}^{(N)}(x) \geqslant 0$ by definition, it is only the last inequality of (4.29) that remains to be proved.

LEMmA 4.5. - Let $d>4$ and assume the bounds (4.17)-(4.19) for $0 \leqslant m \leqslant n+1$. There are constants $C$ and $L_{0}$ (both depending on $K_{f}$ ), such that for $L \geqslant L_{0}, N \geqslant 0$, $q \in\{0,2,4\}$ and $2 \leqslant m \leqslant n+1$,

$$
0 \leqslant \sum_{x}|x|^{q} \pi_{m}^{(N)}(x) \leqslant \sum_{x}|x|^{q} P_{m}^{(N)}(x) \leqslant C^{N} \sigma^{q} \beta^{N \vee 1} m^{(q-d) / 2} .
$$


Before proving Lemma 4.5, we first state and prove a lemma that explains the effect of applying construction 1,2 or $E$ to a diagram. This will be useful in estimating the diagrams $P^{(N)}(\boldsymbol{x})$, which are obtained by repeated application of construction $E$.

Lemma 4.6. - Let $0 \leqslant l \leqslant m \leqslant n+1$ with $m \geqslant 1$, and assume (4.17)-(4.19) for $0 \leqslant m \leqslant n+1$. Let $G$ and $F_{m}(x)$ be diagrams with all vertices having temporal component respectively no larger than $n+1$ and $m$. Suppose that $G$ and $\sum_{x} F_{m}(x)$ can be bounded respectively by $B$ and $B(m+1)^{-d / 2}$ by associating $l^{1}$ and $l^{\infty}$ norms to diagram lines as in Example 4.4 and by using (4.17)-(4.19) to estimate these norms. Then the following statements hold.

(a) Application of construction $1_{l}(\lambda)$ to $G$ produces a diagram that is bounded by $2^{(d+2) / 2} K B$, where $K$ is the constant of Lemma 4.3 .

(b) The diagram $\sum_{x} \widetilde{F}_{m}^{(i)}(x)$, obtained by applying construction $2_{(u, k)}^{(i)}(x, m)$ to $F_{k}(u)$, is bounded by $C \beta^{i} B(m+1)^{-d / 2}$, with $C$ independent of $F_{k}(u)$ and $B$ except for dependence on the number of admissible lines in $F_{k}(u)$.

(c) Application of construction $E_{(u, k)}(x, m)$ to $F_{k}(u)$, followed by summation over $x$, produces a result that is bounded above by $C \beta^{i} B(m+1)^{-d / 2}$, with $C$ independent of $F_{k}(u)$ and $B$ except for dependence on the number of admissible lines in $F_{k}(u)$.

In each of (a)-(c), the bound on the new diagram is obtained by associating $l^{1}$ and $l^{\infty}$ norms to diagram lines as in Example 4.4 and by using (4.17)-(4.19) to estimate these norms.

Proof. - (a) Application of construction $1_{l}(\lambda)$ replaces a factor $\tau_{j}(x)$ associated to line $\lambda$ by a factor $\rho_{j}(x)=\sum_{w} \tau_{l}(w) \tau_{j-l}(x-w)$. By $(4.17),\left\|\rho_{j}\right\|_{1} \leqslant\left\|\tau_{l}\right\|_{1}\left\|\tau_{j-l}\right\|_{1} \leqslant K^{2}$, compared to $\left\|\tau_{j}\right\|_{1} \leqslant K$. In addition, $\left\|\rho_{j}\right\|_{\infty} \leqslant 2^{(d+2) / 2} K^{2} \beta^{1 \vee j}(j+1)^{-d / 2}$, compared to $\left\|\tau_{j}\right\|_{\infty} \leqslant K \beta^{1 \vee j}(j+1)^{-d / 2}$. To see this, we assume first that $l \leqslant j / 2$, in which case $j-l \geqslant j / 2$. In this case, by (4.19), we have

$$
\rho_{j}(x) \leqslant\left\|\tau_{j-l}\right\|_{\infty}\left\|\tau_{l}\right\|_{1} \leqslant 2^{d / 2} K^{2} \beta^{1 \vee j}(j+1)^{-d / 2} .
$$

The case $l>j / 2$ obeys the same bound. Thus the effect of replacing a diagram line $\tau_{j}(x)$ by $\rho_{j}(x)$ is to obtain, at worst, an additional factor $2^{(d+2) / 2} K$ in a bound.

(b) We first show that

$$
\sup _{u, z} \sum_{x} L_{k, j, m}^{(i)}(u, z, x) \leqslant 2^{(d+2) / 2} K^{3} \beta^{i}(m-j+1)^{-d / 2} \quad(j \leqslant k) .
$$

Consider first the case $i=0$, for which the left side of (4.31) is given by

$$
\sup _{u, z} \sum_{x} \tau_{m-k}(x-u) \tau_{m-j}(x-z) .
$$

Since $k$ is the greatest temporal vertex in $F_{k}(u)$ by assumption, we may assume that $j \leqslant$ $k$. In this case, by (4.17)-(4.19) the above expression is bounded by $\left\|\tau_{m-j}\right\|_{\infty}\left\|\tau_{m-k}\right\|_{1} \leqslant$ $K^{2}(m-j+1)^{-d / 2}$, which is better than required. The desired bound for $i=1$ follows from (a) and the observation that $L_{k, j, m}^{(1)}(u, z, x)$ is obtained from $L_{k, j, m}^{(0)}(u, z, x)$ by applying construction $1^{\lambda}(k+1)$, where $\lambda$ is the line $\tau_{m-k}(x-u)$. The factor $\beta$ arises 
because both $m-j \geqslant 1$ and $m-k \geqslant 1$ for $L^{(1)}$ and this ensures that a factor $\beta$ is produced from the $l^{\infty}$ norm in bounding (4.32).

By (4.15),

$$
\sum_{x} \widetilde{F}_{m}^{(i)}(x) \leqslant \sum_{\lambda} \sum_{0 \leqslant j \leqslant k \leqslant m}\left(\sum_{u, z} F_{k}\left(u ; 1^{\lambda}(z, j)\right)\right)\left(\sup _{u, z} \sum_{x} L_{k, j, m}^{(i)}(u, z, x)\right) .
$$

By (a), there is a constant $c_{1}$ such that

$$
\sum_{u, z} F_{k}\left(u ; 1^{\lambda}(z, j)\right) \leqslant c_{1} B(k+1)^{-d / 2}
$$

Using this with (4.31), we see that (4.33) is bounded above by

$$
c_{2} \beta^{i} B \sum_{0 \leqslant j \leqslant k \leqslant m}(k+1)^{-d / 2}(m-j+1)^{-d / 2} .
$$

By (4.26), this gives the desired result.

(c) This follows from (b), since construction $E_{(y, k)}(x, m)$ is obtained by application of construction $2^{(1)}$ followed by construction $2^{(0)}$.

Proof of Lemma 4.5. - In view of the observation above the statement of the lemma, it suffices to show that

$$
\sum_{x}|x|^{q} P_{m}^{(N)}(x) \leqslant C^{N} \sigma^{q} \beta^{N \vee 1}(m+1)^{(q-d) / 2} \quad(N \geqslant 0) .
$$

We first consider the case $q=0$. By definition, for $m \geqslant 1$,

$$
\sum_{x} P_{m}^{(0)}(x)=\sum_{x} \tau_{m}(x)^{2} \leqslant\left\|\tau_{m}\right\|_{\infty}\left\|\tau_{m}\right\|_{1}
$$

The case $N=0, q=0$ of (4.36) then follows immediately from (4.17)-(4.19). The result for general $N$ (with $q=0$ ) then follows from Lemma 4.6(c) and the fact that $P^{(N)}(\boldsymbol{x})$ is obtained from the bubble diagram $B(\boldsymbol{x})=\delta_{\mathbf{0}, \boldsymbol{x}}+P^{(0)}(\boldsymbol{x})$ by $N$ applications of construction $E$.

For $q=2,4$ we note from Fig. 7 that there are two disjoint paths starting at $(0,0)$ leading to $(x, m)$, for each $P^{(N)}$. The number of lines constituting each of these paths is at most $2 N+1$. Denoting the displacements along each of these lines by $u_{i}$ and $v_{i}$, we have $x=\sum_{i} u_{i}=\sum_{i} v_{i}$. Therefore

$$
|x|^{2} \leqslant(2 N+1) \sum_{i}\left|u_{i}\right|^{2}, \quad|x|^{2} \leqslant(2 N+1) \sum_{i}\left|v_{i}\right|^{2} .
$$

Estimating the resulting diagrams term by term and arguing as in Example 4.4, we see that the effect of the factor $|x|^{q}$ is to multiply the bound on the case $q=0$ by $\mathcal{O}\left(N^{2}\right) \sigma^{q} m^{q / 2} \leqslant C^{N} \sigma^{q} m^{q / 2}$. 


\subsection{Proof of Proposition 2.2(i)-(ii)}

In this section, we prove Proposition 2.2(i)-(ii).

Proof of Proposition 2.2(i). - Note that (4.17)-(4.19) for $0 \leqslant m \leqslant n+1$ follow from (2.15) for $1 \leqslant m \leqslant n$, by Lemma 4.3. The desired result then follows immediately from Lemma 4.5 , since $\pi_{m}(x)=\sum_{N=0}^{\infty}(-1)^{N} \pi_{m}^{(N)}(x)$.

Proof of Proposition 2.2(ii). - The following proof is the same as the proof of [26, Proposition 5.1(ii)].

We give separate arguments for $\|k\|_{\infty} \leqslant L^{-1}$ and $\|k\|_{\infty} \geqslant L^{-1}$. For $\|k\|_{\infty} \geqslant L^{-1}$, it follows from Proposition 2.2(i) and (1.5) that for $2 \leqslant m \leqslant n+1$

$$
\begin{aligned}
& \left|\hat{\pi}_{m}(k)-\hat{\pi}_{m}(0)-[1-\widehat{D}(k)] \sigma^{-2} \nabla^{2} \hat{\pi}_{m}(0)\right| \\
& \quad \leqslant \frac{C \beta}{m^{d / 2}}+\frac{C \beta}{m^{d / 2}}+\frac{C \beta[1-\widehat{D}(k)]}{m^{(d-2) / 2}} \leqslant \frac{C \beta[1-\widehat{D}(k)]}{m^{(d-2) / 2}} \leqslant \frac{C \beta[1-\widehat{D}(k)]^{2}}{m^{(d-2) / 2}},
\end{aligned}
$$

by changing the constant $C$ appropriately. This contribution satisfies (2.22).

Henceforth, we restrict attention to $\|k\|_{\infty} \leqslant L^{-1}$. By the triangle inequality,

$$
\begin{aligned}
& \left|\hat{\pi}_{m}(k)-\hat{\pi}_{m}(0)-[1-\widehat{D}(k)] \sigma^{-2} \nabla^{2} \hat{\pi}_{m}(0)\right| \\
& \quad \leqslant\left|\hat{\pi}_{m}(k)-\hat{\pi}_{m}(0)-\frac{|k|^{2}}{2 d} \nabla^{2} \hat{\pi}_{m}(0)\right|+\left|[1-\widehat{D}(k)] \sigma^{-2}-\frac{|k|^{2}}{2 d}\right|\left|\nabla^{2} \hat{\pi}_{m}(0)\right| .
\end{aligned}
$$

By symmetry, the first term on the right side of (4.40) can be rewritten using

$$
\hat{\pi}_{m}(k)-\hat{\pi}_{m}(0)-\frac{|k|^{2}}{2 d} \nabla^{2} \hat{\pi}_{m}(0)=\sum_{x}\left(\cos (k \cdot x)-1+\frac{(k \cdot x)^{2}}{2}\right) \pi_{m}(x) .
$$

There is a constant $c>0$ such that $\left|\cos t-1+\frac{1}{2} t^{2}\right| \leqslant c t^{2+2 \varepsilon^{\prime}}$ for all $\varepsilon^{\prime} \in[0, \varepsilon \wedge 1]$. Since $|k \cdot x|^{2+2 \varepsilon^{\prime}} \leqslant|k|^{2+2 \varepsilon^{\prime}}|x|^{2+2 \varepsilon^{\prime}}$, it follows that

$$
\left|\hat{\pi}_{m}(k)-\hat{\pi}_{m}(0)-\frac{|k|^{2}}{2 d} \nabla^{2} \hat{\pi}_{m}(0)\right| \leqslant c|k|^{2+2 \varepsilon^{\prime}} \sum_{x}|x|^{2+2 \varepsilon^{\prime}}\left|\pi_{m}(x)\right| .
$$

By Hölder's inequality and Proposition 2.2(i) with $q=0,4$,

$$
\sum_{x}|x|^{2+2 \varepsilon^{\prime}}\left|\pi_{m}(x)\right| \leqslant\left(\sum_{x}\left|\pi_{m}(x)\right|\right)^{\frac{1-\varepsilon^{\prime}}{2}}\left(\sum_{x}|x|^{4}\left|\pi_{m}(x)\right|\right)^{\frac{1+\varepsilon^{\prime}}{2}} \leqslant \frac{K \beta \sigma^{2+2 \varepsilon^{\prime}}}{m^{\left(d-2-2 \varepsilon^{\prime}\right) / 2}} .
$$

The desired bound on the first term of (4.40) then follows by combining (4.42) and (4.43) with the lower bound of (1.4). 
It follows from (1.2) and Hölder's inequality that $\sum_{x}|x|^{2+2 \varepsilon^{\prime}} D(x) \leqslant C L^{2+2 \varepsilon^{\prime}}$ for each $\varepsilon^{\prime} \in[0, \varepsilon]$. By Proposition 2.2(i) with $q=2$, arguing as above and using (1.4), it therefore follows that the second term of (4.40) is bounded by

$$
\frac{K \beta}{m^{(d-2) / 2}}\left|[1-\widehat{D}(k)]-\frac{|k|^{2} \sigma^{2}}{2 d}\right| \leqslant \frac{K \beta|k|^{2+2 \varepsilon^{\prime}} L^{2+2 \varepsilon^{\prime}}}{m^{(d-2) / 2}} \leqslant \frac{K \beta}{m^{(d-2) / 2}}[1-\widehat{D}(k)]^{1+\varepsilon^{\prime}},
$$

which satisfies (2.22).

\subsection{Proof of Proposition 2.2(iii)}

In this section, we prove Proposition 2.2(iii), using the Nguyen-Yang expansion of Section 3.2. To begin, we recall from (3.26) the decomposition

$$
\tau_{n}(x)=\sum_{t=0}^{n} \tau_{n, t}(x)
$$

and we recall from (3.36) that

$$
\hat{\pi}_{m}(k)=\sum_{t=0}^{m} \hat{\pi}_{m, t}(k)
$$

The following lemma provides an appealing formula and bound for $p \partial_{p} \hat{\pi}_{n}(k)$.

LEMMA 4.7. - For $p \in[0,1]$ and $d \geqslant 1$, the following formula and bound hold:

$$
\begin{gathered}
p \partial_{p} \hat{\pi}_{n}(k)=\sum_{t=0}^{n} t \hat{\pi}_{n, t}(k), \\
p\left|\partial_{p} \hat{\pi}_{n}(k)\right| \leqslant n \sum_{N=0}^{n} \widehat{\Pi}_{n}^{(N)}(0) .
\end{gathered}
$$

Proof. - By Russo's formula (see [18]),

$$
\partial_{p} \tau_{n}(x)=\frac{1}{p} \sum_{t=0}^{n} t \tau_{n, t}(x) .
$$

Taking the Fourier transform of (3.35) gives

$$
\hat{\tau}_{n+1, t+1}(k)=p \widehat{D}(k) \hat{\tau}_{n, t}(k)+p \widehat{D}(k) \sum_{m=2}^{n} \sum_{s=0}^{t} \hat{\pi}_{m, s}(k) \hat{\tau}_{n-m, t-s}(k)+\hat{\pi}_{n+1, t+1}(k) .
$$

Combining (4.49)-(4.50), we obtain

$$
\begin{aligned}
\partial_{p} \hat{\tau}_{n+1}(k)= & \frac{1}{p} \sum_{t=0}^{n}(t+1)\left[p \widehat{D}(k) \hat{\tau}_{n, t}(k)\right. \\
& \left.+p \widehat{D}(k) \sum_{m=2}^{n} \sum_{s=0}^{t} \hat{\pi}_{m, s}(k) \hat{\tau}_{n-m, t-s}(k)+\hat{\pi}_{n+1, t+1}(k)\right] .
\end{aligned}
$$


We evaluate the contributions due to the two terms in the factor $(t+1)$ separately. By (2.7), (4.45) and (4.46), the term 1 in the factor $(t+1)$ contributes $p^{-1}\left[\hat{\tau}_{n+1}(k)-\right.$ $\left.\hat{\pi}_{n+1,0}(k)\right]$. The contribution due to the term $t$ in the factor $(t+1)$ can be evaluated with the help of (4.45) and (4.49), leading after calculation to the conclusion that

$$
\begin{aligned}
\partial_{p} \hat{\tau}_{n+1}(k)= & \frac{1}{p}\left[\hat{\tau}_{n+1}(k)-\hat{\pi}_{n+1}(k)\right]+\widehat{D}(k) p \partial_{p} \hat{\tau}_{n}(k)+\widehat{D}(k) \sum_{m=2}^{n} \hat{\pi}_{m}(k) p \partial_{p} \hat{\tau}_{n-m}(k) \\
& +\widehat{D}(k) \sum_{m=2}^{n} \sum_{s=0}^{m} s \hat{\pi}_{m, s}(k) \hat{\tau}_{n-m}(k)+\frac{1}{p} \sum_{s=0}^{n+1} s \hat{\pi}_{n+1, s}(k) .
\end{aligned}
$$

On the other hand, differentiating (2.7) with respect to $p$ gives

$$
\begin{aligned}
\partial_{p} \hat{\tau}_{n+1}(k)= & \frac{1}{p}\left[\hat{\tau}_{n+1}(k)-\hat{\pi}_{n+1}(k)\right]+p \widehat{D}(k) \partial_{p} \hat{\tau}_{n}(k)+p \widehat{D}(k) \sum_{m=2}^{n} \hat{\pi}_{m}(k) \partial_{p} \hat{\tau}_{n-m}(k) \\
& +p \widehat{D}(k) \sum_{m=2}^{n} \partial_{p} \hat{\pi}_{m}(k) \hat{\tau}_{n-m}(k)+\partial_{p} \hat{\pi}_{n+1}(k) .
\end{aligned}
$$

Comparing (4.52) and (4.53), we see that

$$
\begin{aligned}
& \widehat{D}(k) \sum_{m=2}^{n} p \partial_{p} \hat{\pi}_{m}(k) \hat{\tau}_{n-m}(k)+\partial_{p} \hat{\pi}_{n+1}(k) \\
& =\widehat{D}(k) \sum_{m=2}^{n} \sum_{s=0}^{m} s \hat{\pi}_{m, s}(k) \hat{\tau}_{n-m}(k)+\frac{1}{p} \sum_{s=0}^{n+1} s \hat{\pi}_{n+1, s}(k) .
\end{aligned}
$$

We will now prove by induction on $n$ that the derivative of $\hat{\pi}_{n}(k)$ is given simply by

$$
p \partial_{p} \hat{\pi}_{n}(k)=\sum_{s=0}^{n} s \hat{\pi}_{n, s}(k)
$$

as claimed in (4.47). For $n=1$, we have $\hat{\pi}_{1}(k)=0$ and $\hat{\pi}_{1, s}(k)=0$ for all $s$, in agreement with (4.55). Next, assume that $p \partial_{p} \hat{\pi}_{m}(k)=\sum_{s=0}^{m} s \hat{\pi}_{m, s}(k)$ for all $m \leqslant n$. The advancement of the induction then follows from (4.54).

Therefore

$$
\left|p \partial_{p} \hat{\pi}_{n}(k)\right| \leqslant n \sum_{s=0}^{n}\left|\hat{\pi}_{n, s}(k)\right| \leqslant n \sum_{N=0}^{\infty} \sum_{s=0}^{n} \widehat{\Pi}_{n, s}^{(N)}(0)=n \sum_{N=0}^{\infty} \widehat{\Pi}_{n}^{(N)}(0),
$$

where we have used (4.55), (3.38), (3.40), and, in the second inequality, the fact that $\Pi_{n, s}^{(N)}(x) \geqslant 0$ for every $n, s, x, N$.

In view of (4.48), to complete the proof of Proposition 2.2(iii) it suffices to show that the estimates (4.17)-(4.19) (which follow from (2.15) by Lemma 4.3) imply that

$$
\widehat{\Pi}_{n}^{(N)}(0) \leqslant C^{N} \beta^{N \vee 1}(n+1)^{-d / 2} \quad(N \geqslant 0) .
$$

This is immediate for $N=0$ by Lemma 4.5 , since $\widehat{\Pi}_{n}^{(0)}(0)=\hat{\pi}_{n, 0}(k)=\hat{\pi}_{n}^{(0)}(0)$ by definition. For $N \geqslant 1$, upper bounds on $\Pi_{n}^{(N)}(x)$ in terms of diagrams are discussed 

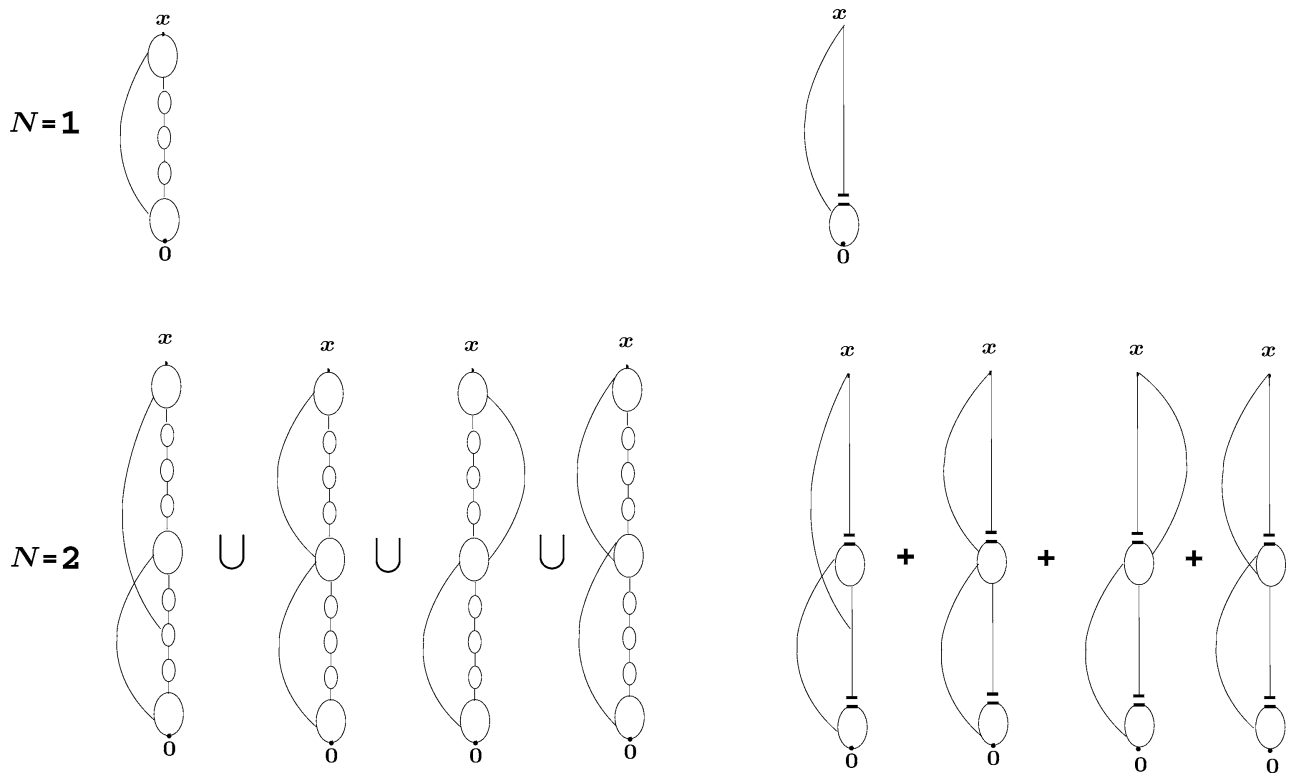

Fig. 8. Disjoint connections occurring in $\Pi_{n}^{(N)}(x)$, and corresponding diagrammatic upper bounds, for $N=1,2$.

in [31]. The diagrams that arise are almost identical in structure to the diagrams that were shown to bound $\pi_{n}^{(N)}(x)$ in Section 4.1.1. Using the definition of $\Pi_{n}^{(N)}(x)$ in (3.39), it can be argued as in [31] that configurations contributing to $\Pi_{n}^{(N)}(x)$ must contain the disjoint connections depicted in Fig. 8, for $N=1,2$. Higher values of $N$ have a similar pattern. In Fig. 8, upper bounds on $\Pi_{n}^{(N)}(x)$ are also depicted, for $N=1,2$. The diagram for $N=1$ is precisely the diagram estimated in Example 4.4. The diagrams for $N \geqslant 2$ can be estimated using construction 2 in a similar fashion to the proof of Lemma 4.5. We omit the details, which lead to the conclusion that (4.57) indeed holds.

\section{Expansion for the $r$-point function}

In this section we derive the expansion (2.29) for the $r$-point function, for $r \geqslant 3$. The derivation also applies when $r=2$, in which case it reproduces the expansion for the two-point function of Section 3.1.

Two approaches to the derivation of (2.29) are possible. One approach is to begin with the expansion for the two-point function with magnetic field developed in [23, Section 4]. Although the expansion with magnetic field was derived in [23] for nonoriented percolation, it applies also in the oriented context without modification. When the expansion of [23, Section 4] is differentiated $r-2$ times with respect to the (sitedependent) magnetic field and the magnetic field is then set equal to zero, an expansion for the $r$-point function results. However, the magnetic field introduces subtleties that are not required for our present purposes, and we find it simpler and more direct to use a different approach that does not employ a magnetic field. This second approach adapts the expansion of Section 3.1 to deal with $r \geqslant 3$ directly. 
In this adaptation, the string of sausages of Fig. 2 of Section 2.2.1 is replaced by the tree of sausages of Fig. 3 of Section 2.2. We regard the tree of sausages as a string of sausages joining $\mathbf{0}$ to $\boldsymbol{x}_{1}$, with branches emerging from the sausages and leading to $\boldsymbol{x}_{2}, \ldots, \boldsymbol{x}_{r-1}$. The point $\boldsymbol{x}_{1}$ therefore plays a distinguished role in the expansion. The derivation of the expansion shares many common features with the derivation of the Hara-Slade expansion of Section 3.1, but contains additional features when $r>2$. A central feature of the expansion is the location of a suitable cutting bond, and, like the cutting bond defined above (3.15), it will be important that we define the cutting bond to be the first bond having a desirable property.

Using the definitions $J=\{1,2, \ldots, r-1\}$ and $J_{1}=J \backslash\{1\}$ of (2.28), the identity (2.29) asserts that

$$
\tau^{(r)}\left(\overrightarrow{\boldsymbol{x}}_{J}\right)=A^{(r)}\left(\overrightarrow{\boldsymbol{x}}_{J}\right)+\sum_{I \subset J_{1}} \sum_{\boldsymbol{v}_{1}} B^{\left(r_{2}+1\right)}\left(\boldsymbol{v}_{1}, \overrightarrow{\boldsymbol{x}}_{I}\right) \tau^{\left(r_{1}\right)}\left(\overrightarrow{\boldsymbol{x}}_{J \backslash I}-\boldsymbol{v}_{1}\right),
$$

where $r_{1}=|J \backslash I|+1$ and $r_{2}=|I|+1$. In what follows, we suppress the superscript $(r)$ from the notation, as this superscript can be determined from the number of vertices appearing as arguments. We will again use the notation $\boldsymbol{x}$ for sites, rather than $(x, n)$. The expansion will be performed in iterative stages, which will lead to expressions for $A$ and $B$ of the form

$$
A\left(\overrightarrow{\boldsymbol{x}}_{J}\right)=\sum_{N=0}^{\infty}(-1)^{N} A^{(N)}\left(\overrightarrow{\boldsymbol{x}}_{J}\right), \quad B\left(\boldsymbol{v}, \overrightarrow{\boldsymbol{x}}_{I}\right)=\sum_{N=0}^{\infty}(-1)^{N} B^{(N)}\left(\boldsymbol{v}, \overrightarrow{\boldsymbol{x}}_{I}\right) .
$$

The superscript $(N)$ in (5.2) should not be confused with the superscript $(r)$ denoting $r$ points in (5.1), and it should be clear from the context which superscript is intended.

Given $I \subset J$, we define

$$
F\left(\boldsymbol{v}, \overrightarrow{\boldsymbol{x}}_{I}\right)=\left\{\boldsymbol{v} \rightarrow \boldsymbol{x}_{i} \text { for all } i \in I\right\} .
$$

Using this notation, the $r$-point function can be written as

$$
\tau\left(\overrightarrow{\boldsymbol{x}}_{J}\right)=\mathbb{P}\left(F\left(\mathbf{0}, \overrightarrow{\boldsymbol{x}}_{J}\right)\right) .
$$

We also define

$$
A^{(0)}\left(\overrightarrow{\boldsymbol{x}}_{J}\right)=\mathbb{P}\left(\left(\mathbf{0} \Rightarrow \boldsymbol{x}_{1}\right) \cap F\left(\mathbf{0}, \overrightarrow{\boldsymbol{x}}_{J_{1}}\right)\right) .
$$

Since $\mathbf{0}$ is either doubly connected to $\boldsymbol{x}_{1}$ or it is not, we have

$$
\tau\left(\overrightarrow{\boldsymbol{x}}_{J}\right)=A^{(0)}\left(\overrightarrow{\boldsymbol{x}}_{J}\right)+\mathbb{P}\left(\left\{\mathbf{0} \rightarrow \boldsymbol{x}_{1} \& \mathbf{0} \nRightarrow \boldsymbol{x}_{1}\right\} \cap F\left(\mathbf{0}, \overrightarrow{\boldsymbol{x}}_{J_{1}}\right)\right) .
$$

If $\mathbf{0}$ is connected but not doubly connected to $\boldsymbol{x}_{1}$, then there is a first pivotal bond $(\boldsymbol{u}, \boldsymbol{v})$ for the connection and hence

$$
\begin{aligned}
& \mathbb{P}\left(\left\{\mathbf{0} \rightarrow \boldsymbol{x}_{1} \& \mathbf{0} \nRightarrow \boldsymbol{x}_{1}\right\} \cap F\left(\mathbf{0}, \overrightarrow{\boldsymbol{x}}_{J_{1}}\right)\right) \\
& \quad=\sum_{(\boldsymbol{u}, \boldsymbol{v})} \mathbb{P}\left(\left\{\mathbf{0} \Rightarrow \boldsymbol{u} \&(\boldsymbol{u}, \boldsymbol{v}) \text { is occupied and pivotal for } \mathbf{0} \rightarrow \boldsymbol{x}_{1}\right\} \cap F\left(\mathbf{0}, \overrightarrow{\boldsymbol{x}}_{J_{1}}\right)\right) .
\end{aligned}
$$


Regarding the connection $\mathbf{0} \rightarrow \boldsymbol{x}_{1}$ as a string of sausages as we did in Section 3.1, we partition the event on the right side of (5.7) according to the set $I_{1} \subset J_{1}$ such that $\overrightarrow{\boldsymbol{x}}_{I_{1}}$ lies in the first sausage and $\overrightarrow{\boldsymbol{x}}_{J \backslash I_{1}}$ does not. In other words, $I_{1}$ is the largest set such that $\overrightarrow{\boldsymbol{x}}_{I_{1}} \subset \widetilde{C}^{(\boldsymbol{u}, \boldsymbol{v})}(\mathbf{0})$. Defining

$$
F^{\prime \prime}\left(\boldsymbol{u}, \boldsymbol{v}, \overrightarrow{\boldsymbol{x}}_{I_{1}}\right)=\left\{\left\{(\mathbf{0} \Rightarrow \boldsymbol{u}) \cap F\left(\mathbf{0}, \overrightarrow{\boldsymbol{x}}_{I_{1}}\right)\right\} \text { occurs on } \widetilde{C}^{(\boldsymbol{u}, \boldsymbol{v})}(\mathbf{0})\right\},
$$

we claim that, as in (3.10),

$\mathbb{P}\left(\{\mathbf{0} \Rightarrow \boldsymbol{u}\} \&\left\{(\boldsymbol{u}, \boldsymbol{v})\right.\right.$ is occupied and pivotal for $\left.\left.\mathbf{0} \rightarrow \boldsymbol{x}_{1}\right\} \cap F\left(\mathbf{0}, \overrightarrow{\boldsymbol{x}}_{J_{1}}\right)\right)$

$=\sum_{I_{1} \subset J_{1}} \mathbb{P}\left(F^{\prime \prime}\left(\boldsymbol{u}, \boldsymbol{v}, \overrightarrow{\boldsymbol{x}}_{I_{1}}\right) \cap\{(\boldsymbol{u}, \boldsymbol{v})\right.$ occupied $\} \cap\left\{F\left(\boldsymbol{v}, \overrightarrow{\boldsymbol{x}}_{J \backslash I_{1}}\right)\right.$ occurs in $\left.\left.\Lambda \backslash \widetilde{C}^{(\boldsymbol{u}, \boldsymbol{v})}(\boldsymbol{0})\right\}\right)$.

To prove (5.9), we partition the event on the left side according to the set $I_{1}$ of components of $\overrightarrow{\boldsymbol{x}}_{J}$ that are in $\widetilde{\boldsymbol{C}}^{(\boldsymbol{u}, \boldsymbol{v})}(\mathbf{0})$, obtaining a disjoint union of events that we denote in this paragraph as $E_{I_{1}}$. Given $I_{1}$, we show that $E_{I_{1}}$ is equal to the event appearing on the right side of (5.9). To show that $E_{I_{1}}$ is contained in the event of the right side, by definition of $E_{I_{1}}$ it follows that $F^{\prime \prime}\left(\boldsymbol{u}, \boldsymbol{v}, \overrightarrow{\boldsymbol{x}}_{I_{1}}\right)$ occurs, that $(\boldsymbol{u}, \boldsymbol{v})$ is occupied, and that $F\left(\boldsymbol{v}, \overrightarrow{\boldsymbol{x}}_{J \backslash I_{1}}\right)$ occurs. Thus it suffices to verify that $F\left(\boldsymbol{v}, \overrightarrow{\boldsymbol{x}}_{J \backslash I_{1}}\right)$ occurs in $\Lambda \backslash \widetilde{\boldsymbol{C}}^{(\boldsymbol{u}, \boldsymbol{v})}(\mathbf{0})$. To see that $F\left(\boldsymbol{v}, \overrightarrow{\boldsymbol{x}}_{J \backslash I_{1}}\right)$ occurs in $\Lambda \backslash \widetilde{C}^{(\boldsymbol{u}, \boldsymbol{v})}(\mathbf{0})$, we observe that otherwise $\boldsymbol{v}$ must be connected to some $\boldsymbol{x}_{i}$ (with $i \in J \backslash I_{1}$ ) through $\widetilde{C}^{(\boldsymbol{u}, \boldsymbol{v})}(\mathbf{0})$. But this contradicts either the fact that $(\boldsymbol{u}, \boldsymbol{v})$ is pivotal for $\mathbf{0} \rightarrow \boldsymbol{x}_{1}$ (if $i=1$ ), or the fact that $i \notin I_{1}$ (if $i \neq 1$ ). Conversely, suppose that the event of the right side of (5.9) occurs. Then it is clear that all the events in the definition of $E_{I_{1}}$ occur, except it is not immediately obvious that $(\boldsymbol{u}, \boldsymbol{v})$ must be pivotal for $\mathbf{0} \rightarrow \boldsymbol{x}_{1}$. However, if $(\boldsymbol{u}, \boldsymbol{v})$ is not pivotal, then $\boldsymbol{x}_{1} \in \widetilde{\boldsymbol{C}}^{(\boldsymbol{u}, \boldsymbol{v})}(0)$, which contradicts the fact that $F\left(\boldsymbol{v}, \overrightarrow{\boldsymbol{x}}_{J \backslash I_{1}}\right)$ occurs in $\Lambda \backslash \widetilde{C}^{(\boldsymbol{u}, \boldsymbol{v})}(\mathbf{0})$. This completes the proof of (5.9).

Given $A \subset \Lambda$, we define a restricted connectivity function by

$$
\tau^{A}\left(\boldsymbol{v}, \overrightarrow{\boldsymbol{x}}_{I}\right)=\mathbb{P}\left(\boldsymbol{v} \rightarrow \boldsymbol{x}_{i} \text { occurs in } \Lambda \backslash A \text { for each } i \in I\right) .
$$

Applying Lemma 3.3 to the summand in (5.9), we obtain

$$
\begin{aligned}
& \mathbb{P}\left(F^{\prime \prime}\left(\boldsymbol{u}, \boldsymbol{v}, \overrightarrow{\boldsymbol{x}}_{I_{1}}\right) \cap\{(\boldsymbol{u}, \boldsymbol{v}) \text { occupied }\} \cap\left\{F\left(\boldsymbol{v}, \overrightarrow{\boldsymbol{x}}_{J \backslash I_{1}}\right) \text { occurs in } \Lambda \backslash \widetilde{C}^{(\boldsymbol{u}, \boldsymbol{v})}(\mathbf{0})\right\}\right) \\
& \quad=p D(\boldsymbol{v}-\boldsymbol{u}) \mathbb{E}\left(I\left[F^{\prime \prime}\left(\boldsymbol{u}, \boldsymbol{v}, \overrightarrow{\boldsymbol{x}}_{I_{1}}\right)\right] \tau^{\widetilde{C}^{(\boldsymbol{u}, \boldsymbol{v})}(\boldsymbol{0})}\left(\boldsymbol{v}, \overrightarrow{\boldsymbol{x}}_{J \backslash I_{1}}\right)\right) .
\end{aligned}
$$

Let $\boldsymbol{v} \stackrel{A}{\rightarrow} \overrightarrow{\boldsymbol{x}}_{I}$ denote the event that $\boldsymbol{v} \rightarrow \boldsymbol{x}_{i}$ for all $i \in I$ and that $\boldsymbol{v} \stackrel{A}{\rightarrow} \boldsymbol{x}_{i}$ for at least one $i \in I$. By definition,

$$
\begin{aligned}
\tau^{A}\left(\boldsymbol{v}, \overrightarrow{\boldsymbol{x}}_{J \backslash I_{1}}\right) & =\tau\left(\overrightarrow{\boldsymbol{x}}_{J \backslash I_{1}}-\boldsymbol{v}\right)-\left\{\tau\left(\overrightarrow{\boldsymbol{x}}_{J \backslash I_{1}}-\boldsymbol{v}\right)-\tau^{A}\left(\boldsymbol{v}, \overrightarrow{\boldsymbol{x}}_{J \backslash I_{1}}\right)\right\} \\
& =\tau\left(\overrightarrow{\boldsymbol{x}}_{J \backslash I_{1}}-\boldsymbol{v}\right)-\mathbb{P}\left(\boldsymbol{v} \stackrel{A}{\rightarrow} \overrightarrow{\boldsymbol{x}}_{J \backslash I_{1}}\right) .
\end{aligned}
$$

Let

$$
B^{(0)}\left(\boldsymbol{v}, \overrightarrow{\boldsymbol{x}}_{I}\right)=\sum_{\boldsymbol{u}} p D(\boldsymbol{v}-\boldsymbol{u}) \mathbb{P}\left(F^{\prime \prime}\left(\boldsymbol{u}, \boldsymbol{v}, \overrightarrow{\boldsymbol{x}}_{I}\right)\right)
$$


Using (5.7)-(5.13) with (5.6) yields

$$
\begin{aligned}
\tau\left(\overrightarrow{\boldsymbol{x}}_{J}\right)= & A^{(0)}\left(\overrightarrow{\boldsymbol{x}}_{J}\right)+\sum_{I_{1} \subset J_{1}} \sum_{\boldsymbol{v}} B^{(0)}\left(\boldsymbol{v}, \overrightarrow{\boldsymbol{x}}_{I_{1}}\right) \tau\left(\overrightarrow{\boldsymbol{x}}_{J \backslash I_{1}}-\boldsymbol{v}\right) \\
& -\sum_{I_{1} \subset J_{1}(\boldsymbol{u}, \boldsymbol{v})} p D(\boldsymbol{v}-\boldsymbol{u}) \mathbb{E}_{0}\left(I\left[F^{\prime \prime}\left(\boldsymbol{u}, \boldsymbol{v}, \overrightarrow{\boldsymbol{x}}_{I_{1}}\right)\right] \mathbb{P}_{1}\left(\boldsymbol{v} \stackrel{\widetilde{C}_{0}^{(u, v)}(\mathbf{0})}{\longrightarrow} \overrightarrow{\boldsymbol{x}}_{J \backslash I_{1}}\right)\right) .
\end{aligned}
$$

This completes the first stage of the expansion. As in Section 3.1, we have used subscripts in (5.14) to coordinate the cluster $\widetilde{C}_{0}^{(\boldsymbol{u}, \boldsymbol{v})}(\mathbf{0})$ and the expectation $\mathbb{E}_{0}$ with respect to which $\widetilde{C}_{0}^{(\boldsymbol{u}, \boldsymbol{v})}(\mathbf{0})$ is random.

Fix $K \subset J$ with $K \ni 1$, and let $K_{1}=K \backslash\{1\}$. To continue the expansion, we begin by analysing the probability $\mathbb{P}\left(\boldsymbol{v} \stackrel{A}{\rightarrow} \overrightarrow{\boldsymbol{x}}_{K}\right)$ occuring in (5.14). Our first task is to identify a cutting bond analogous to the cutting bond introduced above (3.15). For this, given a configuration for which $\stackrel{\vec{v}}{\rightarrow} \overrightarrow{\boldsymbol{x}}_{K}$, we temporally order the pivotal bonds for the connection $\boldsymbol{v} \rightarrow \boldsymbol{x}_{1}$. The cutting bond for $\boldsymbol{v} \rightarrow \boldsymbol{x}_{1}$ is then defined to be the first pivotal bond $\left(\boldsymbol{u}^{\prime}, \boldsymbol{v}^{\prime}\right)$ for $\boldsymbol{v} \rightarrow \boldsymbol{x}_{1}$ (if there is one) such that either (i) $\boldsymbol{v} \stackrel{A}{\rightarrow} \boldsymbol{u}^{\prime}$ or (ii) $\exists i \in K_{1}$ such that $\left\{\boldsymbol{v} \stackrel{A}{\rightarrow} \boldsymbol{x}_{i}\right\}$ occurs on $\widetilde{\boldsymbol{C}}^{\left(\boldsymbol{u}^{\prime}, \boldsymbol{v}^{\prime}\right)}(\boldsymbol{v})$. Examples of cutting bonds are depicted in Fig. 9.

The cutting bond has been defined in such a way that the cluster $\widetilde{C}^{\left(\boldsymbol{u}^{\prime}, \boldsymbol{v}^{\prime}\right)}(\boldsymbol{v})$ remains appropriately "well connected" to the set $A=\widetilde{C}_{0}^{(\boldsymbol{u}, \boldsymbol{v})}(\mathbf{0})$. The term "well connected" is being used in a vague sense here. Roughly speaking, it means that if connections in both $\widetilde{\boldsymbol{C}}^{\left(\boldsymbol{u}^{\prime}, \boldsymbol{v}^{\prime}\right)}(\boldsymbol{v})$ and $\widetilde{\boldsymbol{C}}_{0}^{(\boldsymbol{u}, \boldsymbol{v})}(\mathbf{0})$ are considered, $\mathbf{0}$ should be doubly connected to the bottom $\boldsymbol{u}^{\prime}$ of the cutting bond. In case (i), depicted in the first configuration in Fig. 9, the choice of cutting bond is quite similar to the cutting bond defined in the expansion for the twopoint function above (3.15). In case (ii), depicted in the second configuration in Fig. 9, we have a situation that does not occur for the expansion of the two-point function. In case (ii), the cluster $\widetilde{C}^{\left(\boldsymbol{u}^{\prime}, \boldsymbol{v}^{\prime}\right)}(\boldsymbol{v})$ is well connected to the set $A=\widetilde{C}_{0}^{(\boldsymbol{u}, \boldsymbol{v})}(\mathbf{0})$ via a branch to some $\boldsymbol{x}_{i}$ with $i \neq 1$. The general philosophy is to select the cutting bond to be the first pivotal bond for the connection $\mathbf{0} \rightarrow \boldsymbol{x}_{1}$ such that the cluster $\widetilde{C}^{\left(\boldsymbol{u}^{\prime}, \boldsymbol{v}^{\prime}\right)}(\boldsymbol{v})$ is well connected
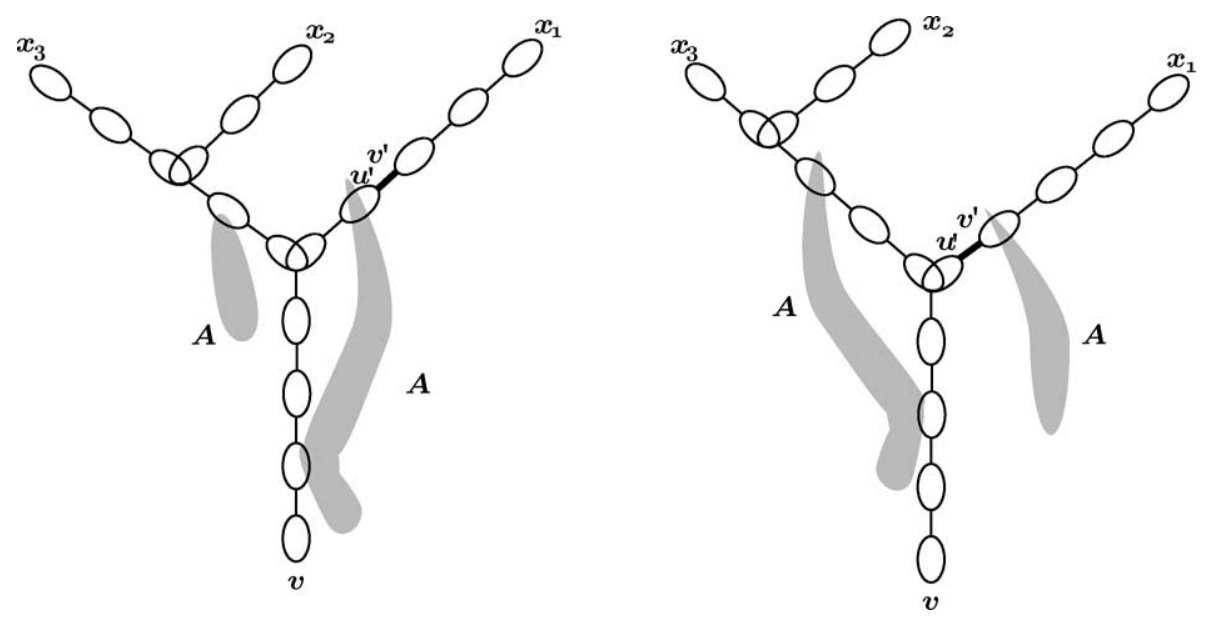

Fig. 9. Examples of cutting bonds $\left(\boldsymbol{u}^{\prime}, \boldsymbol{v}^{\prime}\right)$ for $\boldsymbol{v} \stackrel{A}{\rightarrow} \overrightarrow{\boldsymbol{x}}_{K}$ for $K=\{1,2,3\}$. 
to $A=\widetilde{C}_{0}^{(\boldsymbol{u}, \boldsymbol{v})}(\mathbf{0})$. It is important to choose the first such bond, so that the cluster $C\left(\boldsymbol{v}^{\prime}\right)$ is unconstrained by $A$.

We partition the event $\boldsymbol{v} \stackrel{A}{\rightarrow} \overrightarrow{\boldsymbol{x}}_{K}$ according to the location of the cutting bond, or the absence of a cutting bond. For this, the following notation will be used. Let

$$
\begin{aligned}
G^{\prime}\left(\boldsymbol{v}, \boldsymbol{x}_{1}, \overrightarrow{\boldsymbol{x}}_{K_{1}} ; A\right) & =\left\{\boldsymbol{v} \stackrel{A}{\rightarrow} \overrightarrow{\boldsymbol{x}}_{K}\right\} \cap\left\{\nexists \text { a cutting bond for } \boldsymbol{v} \rightarrow \boldsymbol{x}_{1}\right\}, \\
G^{\prime \prime}\left(\boldsymbol{v}, \boldsymbol{u}^{\prime}, \boldsymbol{v}^{\prime}, \overrightarrow{\boldsymbol{x}}_{K_{1}} ; A\right) & =\left\{G^{\prime}\left(\boldsymbol{v}, \boldsymbol{u}^{\prime}, \overrightarrow{\boldsymbol{x}}_{K_{1}} ; A\right) \text { occurs on } \widetilde{C}^{\left(\boldsymbol{u}^{\prime}, \boldsymbol{v}^{\prime}\right)}(\boldsymbol{v})\right\},
\end{aligned}
$$

where $\boldsymbol{u}^{\prime}$ plays the role of $\boldsymbol{x}_{1}$ in $G^{\prime}\left(\boldsymbol{v}, \boldsymbol{u}^{\prime}, \overrightarrow{\boldsymbol{x}}_{K_{1}} ; A\right)$. Comparing with (3.15) and (3.19), we see that

$$
\begin{aligned}
& G^{\prime}\left(\boldsymbol{v}, \boldsymbol{x}_{1}, \overrightarrow{\boldsymbol{x}}_{\emptyset} ; A\right)=E^{\prime}\left(\boldsymbol{v}, \boldsymbol{x}_{1} ; A\right), \\
& G^{\prime \prime}\left(\boldsymbol{v}, \boldsymbol{u}^{\prime}, \boldsymbol{v}^{\prime}, \overrightarrow{\boldsymbol{x}}_{\emptyset} ; A\right)=E^{\prime \prime}\left(\boldsymbol{v}, \boldsymbol{u}^{\prime}, \boldsymbol{v}^{\prime} ; A\right),
\end{aligned}
$$

which relates the events (5.15)-(5.16) to corresponding events arising in the expansion of Section 3.1. By definition, we have the partition

$$
\begin{aligned}
\left\{\boldsymbol{v} \stackrel{A}{\rightarrow} \overrightarrow{\boldsymbol{x}}_{K}\right\}= & G^{\prime}\left(\boldsymbol{v}, \boldsymbol{x}_{1}, \overrightarrow{\boldsymbol{x}}_{K_{1}} ; A\right) \bigcup_{I \subset K_{1}\left(\boldsymbol{u}^{\prime}, \boldsymbol{v}^{\prime}\right)}\left(G^{\prime}\left(\boldsymbol{v}, \boldsymbol{u}^{\prime}, \overrightarrow{\boldsymbol{x}}_{I} ; A\right)\right. \\
& \cap\left\{\left(\boldsymbol{u}^{\prime}, \boldsymbol{v}^{\prime}\right) \text { occupied and pivotal for } \boldsymbol{v} \rightarrow \boldsymbol{x}_{1}\right\} \\
& \left.\cap F\left(\boldsymbol{v}^{\prime}, \overrightarrow{\boldsymbol{x}}_{K \backslash I}\right)\right) .
\end{aligned}
$$

The event in large parentheses on the right side, consisting of an intersection of three events, can be rewritten as

$$
\begin{aligned}
& G^{\prime \prime}\left(\boldsymbol{v}, \boldsymbol{u}^{\prime}, \boldsymbol{v}^{\prime}, \overrightarrow{\boldsymbol{x}}_{I} ; A\right) \cap\left\{\left(\boldsymbol{u}^{\prime}, \boldsymbol{v}^{\prime}\right) \text { occupied }\right\} \\
& \quad \cap\left\{F\left(\boldsymbol{v}^{\prime}, \overrightarrow{\boldsymbol{x}}_{K \backslash I}\right) \text { occurs in } \Lambda \backslash \widetilde{C}^{\left(\boldsymbol{u}^{\prime}, \boldsymbol{v}^{\prime}\right)}(\boldsymbol{v})\right\} .
\end{aligned}
$$

The proof of (5.19) is similar to the proof of (5.9) and is omitted here. By Lemma 3.3, the probability of the event (5.19) is equal to

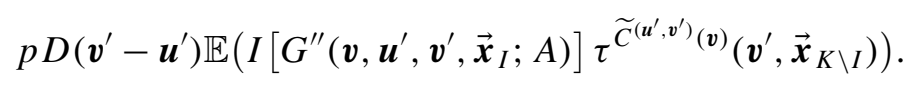

Using an identity corresponding to (5.12), this leads to

$$
\begin{aligned}
\mathbb{P}_{1}\left(\boldsymbol{v} \stackrel{A}{\rightarrow} \overrightarrow{\boldsymbol{x}}_{K}\right)= & \mathbb{P}_{1}\left(G^{\prime}\left(\boldsymbol{v}, \boldsymbol{x}_{1}, \overrightarrow{\boldsymbol{x}}_{K_{1}} ; A\right)\right) \\
& +\sum_{I \subset K_{1}} \sum_{\left(\boldsymbol{u}^{\prime}, \boldsymbol{v}^{\prime}\right)} \mathbb{P}_{1}\left(G^{\prime \prime}\left(\boldsymbol{v}, \boldsymbol{u}^{\prime}, \boldsymbol{v}^{\prime}, \overrightarrow{\boldsymbol{x}}_{I} ; A\right)\right) p D\left(\boldsymbol{v}^{\prime}-\boldsymbol{u}^{\prime}\right) \tau\left(\overrightarrow{\boldsymbol{x}}_{K \backslash I}-\boldsymbol{v}^{\prime}\right) \\
& -\sum_{I \subset K_{1}} \sum_{\left(\boldsymbol{u}^{\prime}, \boldsymbol{v}^{\prime}\right)} p D\left(\boldsymbol{v}^{\prime}-\boldsymbol{u}^{\prime}\right) \\
& \times \mathbb{E}_{1}\left(I\left[G^{\prime \prime}\left(\boldsymbol{v}, \boldsymbol{u}^{\prime}, \boldsymbol{v}^{\prime}, \overrightarrow{\boldsymbol{x}}_{I} ; A\right)\right] \mathbb{P}_{2}\left(\boldsymbol{v}^{\prime} \stackrel{\widetilde{C}_{1}^{\left(\boldsymbol{u}^{\prime}, \boldsymbol{v}^{\prime}\right)}(\boldsymbol{v})}{\longrightarrow} \overrightarrow{\boldsymbol{x}}_{K \backslash I}\right)\right) .
\end{aligned}
$$

Note that (5.21) is well set up for iteration, since a probability of the same form as the left side occurs also on the right side.

We now define 


$$
\begin{aligned}
A^{(1)}\left(\overrightarrow{\boldsymbol{x}}_{J}\right)= & \sum_{I_{1} \subset J_{1}} \sum_{(\boldsymbol{u}, \boldsymbol{v})} p D(\boldsymbol{v}-\boldsymbol{u}) \\
& \times \mathbb{E}_{0}\left(I\left[F^{\prime \prime}\left(\boldsymbol{u}, \boldsymbol{v}, \overrightarrow{\boldsymbol{x}}_{I_{1}}\right)\right] \mathbb{P}_{1}\left(G^{\prime}\left(\boldsymbol{v}, \boldsymbol{x}_{1}, \overrightarrow{\boldsymbol{x}}_{J_{1} \backslash I_{1}} ; \widetilde{C}_{0}^{(\boldsymbol{u}, \boldsymbol{v})}(\mathbf{0})\right)\right)\right), \\
B^{(1)}\left(\boldsymbol{v}^{\prime}, \overrightarrow{\boldsymbol{x}}_{I}\right)= & \sum_{I_{1} \subset I(\boldsymbol{u}, \boldsymbol{v})} \sum_{\boldsymbol{u}^{\prime}} p D(\boldsymbol{v}-\boldsymbol{u}) p D\left(\boldsymbol{v}^{\prime}-\boldsymbol{u}^{\prime}\right) \\
& \times \mathbb{E}_{0}\left(I\left[F^{\prime \prime}\left(\boldsymbol{u}, \boldsymbol{v}, \overrightarrow{\boldsymbol{x}}_{I_{1}}\right)\right] \mathbb{P}_{1}\left(G^{\prime \prime}\left(\boldsymbol{v}, \boldsymbol{u}^{\prime}, \boldsymbol{v}^{\prime}, \overrightarrow{\boldsymbol{x}}_{I \backslash I_{1}} ; \widetilde{C}_{0}^{(\boldsymbol{u}, \boldsymbol{v})}(\mathbf{0})\right)\right)\right),
\end{aligned}
$$

to arrive at

$$
\begin{aligned}
\tau\left(\overrightarrow{\boldsymbol{x}}_{J}\right)= & A^{(0)}\left(\overrightarrow{\boldsymbol{x}}_{J}\right)-A^{(1)}\left(\overrightarrow{\boldsymbol{x}}_{J}\right)+\sum_{I \subset J_{1}} \sum_{\boldsymbol{v}}\left[B^{(0)}\left(\boldsymbol{v}, \overrightarrow{\boldsymbol{x}}_{I}\right)-B^{(1)}\left(\boldsymbol{v}, \overrightarrow{\boldsymbol{x}}_{I}\right)\right] \tau\left(\overrightarrow{\boldsymbol{x}}_{J \backslash I}-\boldsymbol{v}\right) \\
& +\sum_{I_{1} \subset J_{1}} \sum_{I_{2} \subset J_{1} \backslash I_{1}} \sum_{(\boldsymbol{u}, \boldsymbol{v})} \sum_{\left(\boldsymbol{u}^{\prime}, \boldsymbol{v}^{\prime}\right)} p D(\boldsymbol{v}-\boldsymbol{u}) p D\left(\boldsymbol{v}^{\prime}-\boldsymbol{u}^{\prime}\right) \mathbb{E}_{0}\left(I\left[F^{\prime \prime}\left(\boldsymbol{u}, \boldsymbol{v}, \overrightarrow{\boldsymbol{x}}_{I_{1}}\right)\right]\right. \\
& \left.\times \mathbb{E}_{1}\left(I\left[G^{\prime \prime}\left(\boldsymbol{v}, \boldsymbol{u}^{\prime}, \boldsymbol{v}^{\prime}, \overrightarrow{\boldsymbol{x}}_{I_{2}} ; \widetilde{C}_{0}^{(\boldsymbol{u}, \boldsymbol{v})}(\mathbf{0})\right)\right] \mathbb{P}_{2}\left(\boldsymbol{v}^{\prime} \stackrel{\widetilde{C}_{1}^{\left(\boldsymbol{u}^{\prime}, \boldsymbol{v}^{\prime}\right)}(\boldsymbol{v})}{\longrightarrow} \overrightarrow{\boldsymbol{x}}_{J \backslash\left(I_{1} \cup I_{2}\right)}\right)\right)\right) .
\end{aligned}
$$

This completes the second stage of the expansion.

We now repeat this procedure, replacing the probability $\mathbb{P}_{2}$ on the right side of (5.24) using (5.21). This procedure stops after finitely many steps, because each application of (5.21) uses up at least one temporal interval on the way to $\boldsymbol{x}_{1}$, and $\boldsymbol{x}_{1}$ has a finite temporal component. We again use the abbreviation $\widetilde{C}_{j}=\widetilde{C}_{j}^{\left(\boldsymbol{u}_{j}, \boldsymbol{v}_{j}\right)}\left(\boldsymbol{v}_{j-1}\right)$ that was introduced below (3.25). The result is (5.1) and (5.2), with $A^{(0)}$ and $B^{(0)}$ defined in (5.5) and (5.13), and with $A^{(N)}$ and $B^{(N)}$ defined for $N \geqslant 1$ by

$$
\begin{aligned}
A^{(N)}\left(\overrightarrow{\boldsymbol{x}}_{J}\right)= & \sum_{I \subset J_{1}} \sum_{I_{0}, \ldots, I_{N-1}: \bigcup_{i} I_{i}=I} \sum_{\left(\boldsymbol{u}_{0}, \boldsymbol{v}_{0}\right)} \ldots \sum_{\left(\boldsymbol{u}_{N-1}, \boldsymbol{v}_{N-1}\right)}\left[\prod_{i=0}^{N-1} p D\left(\boldsymbol{v}_{i}-\boldsymbol{u}_{i}\right)\right] \\
& \times \mathbb{E}_{0} I\left[F^{\prime \prime}\left(\boldsymbol{u}_{0}, \boldsymbol{v}_{0}, \overrightarrow{\boldsymbol{x}}_{I_{0}}\right)\right] \mathbb{E}_{1} I\left[G^{\prime \prime}\left(\boldsymbol{v}_{0}, \boldsymbol{u}_{1}, \boldsymbol{v}_{1}, \overrightarrow{\boldsymbol{x}}_{I_{1}} ; \widetilde{\boldsymbol{C}}_{0}\right)\right] \ldots \\
& \times \mathbb{E}_{N-1} I\left[G^{\prime \prime}\left(\boldsymbol{v}_{N-2}, \boldsymbol{u}_{N-1}, \boldsymbol{v}_{N-1}, \overrightarrow{\boldsymbol{x}}_{I_{N-1}} ; \widetilde{C}_{N-2}\right)\right] \\
& \times \mathbb{E}_{N} I\left[G^{\prime}\left(\boldsymbol{v}_{N-1}, \overrightarrow{\boldsymbol{x}}_{J \backslash I} ; \widetilde{C}_{N-1}\right)\right], \\
B^{(N)}\left(\boldsymbol{v}, \overrightarrow{\boldsymbol{x}}_{I}\right)= & \sum_{I_{0}, \ldots, I_{N}: \dot{\bigcup}_{i} I_{i}=I} \sum_{\left(\boldsymbol{u}_{0}, \boldsymbol{v}_{0}\right)} \ldots \sum_{\left(\boldsymbol{u}_{N}, \boldsymbol{v}_{N}\right)} \delta_{\boldsymbol{v}_{N}, \boldsymbol{v}}\left[\prod_{i=0}^{N} p D\left(\boldsymbol{v}_{i}-\boldsymbol{u}_{i}\right)\right] \\
& \times \mathbb{E}_{0} I\left[F^{\prime \prime}\left(\boldsymbol{u}_{0}, \boldsymbol{v}_{0}, \overrightarrow{\boldsymbol{x}}_{I_{0}}\right)\right] \mathbb{E}_{1} I\left[G^{\prime \prime}\left(\boldsymbol{v}_{0}, \boldsymbol{u}_{1}, \boldsymbol{v}_{1}, \overrightarrow{\boldsymbol{x}}_{I_{1}} ; \widetilde{C}_{0}\right)\right] \ldots \\
& \times \mathbb{E}_{N} I\left[G^{\prime \prime}\left(\boldsymbol{v}_{N-1}, \boldsymbol{u}_{N}, \boldsymbol{v}_{N}, \overrightarrow{\boldsymbol{x}}_{I_{N}} ; \widetilde{C}_{N-1}\right)\right]
\end{aligned}
$$

The differences between $A^{(N)}$ and $B^{(N)}$ are minor. In $A^{(N)}$ there is a sum over $I$, whereas $I$ is fixed for $B^{(N)}$. In $B^{(N)}$ the event in the $N$ th expectation has a double prime, whereas the corresponding event in $A^{(N)}$ has a single prime. Finally, in $B^{(N)}$ there is an additional sum over $\left(\boldsymbol{u}_{N}, \boldsymbol{v}_{N}\right)$, with $\boldsymbol{v}_{N}$ fixed to equal $\boldsymbol{v}$. 


\section{The second expansion}

In this section, we perform a second expansion to derive the identity (2.30), which states that

$$
B^{(I I \mid+2)}\left(\boldsymbol{v}, \overrightarrow{\boldsymbol{x}}_{I}\right)=\sum_{\boldsymbol{w}} C(\boldsymbol{v}, \boldsymbol{w}) \tau^{(|I|+1)}\left(\overrightarrow{\boldsymbol{x}}_{I}-\boldsymbol{w}\right)+R^{(|I|+2)}\left(\boldsymbol{v}, \overrightarrow{\boldsymbol{x}}_{I}\right) \quad(I \neq \emptyset) .
$$

The left side of (6.1) is defined by (5.2), (5.13) and (5.26). In what follows, we will drop the superscripts on $B$ and $R$ in (6.1), which should not be confused with the superscripts $(N)$ of (5.2) that we will need.

Before going into the details, we give a brief overview of the second expansion. As a first step, we will identify the contribution $b$ to $B$ in which exactly one $I_{i}$ is nonempty as the main term. Thus, in $b$, there is exactly one expectation, say the expectation at level$j$, in which a connection to $\boldsymbol{x}_{I}$ is present. Secondly, for $j<N$ the cluster $\widetilde{C}_{j}$ at level- $j$ plays a role also at level- $(j+1)$. To place all restrictions on the level- $j$ configuration within a single expectation, we will interchange $\mathbb{E}_{j}$ and $\mathbb{E}_{j+1}$. The resulting event at level- $j$ then depends on the configurations at levels- $(j \pm 1)$, and the second expansion takes place on this event. Thirdly, we will need to define a suitable cutting bond for the second expansion. This cutting bond needs to be chosen after all the restrictions placed on the configurations from levels- $(j \pm 1)$ have been satisfied, and early enough that the bottom of the cutting bond remains "well-connected" in a sense related to that discussed in Section 5. Using the cutting bond, we apply the factorisation Lemma 3.3 in the usual way. The details are as follows.

The contribution to $B^{(N)}$ for which more than one $I_{i} \neq \varnothing$ in (5.26) corresponds to branching of degree greater than three. This is neglible in the scaling limit and is an error term that forms part of $R$. Such a contribution can only occur when $|I| \geqslant 2$. We denote the sum over $N$ of these contributions by $c\left(\boldsymbol{v}, \overrightarrow{\boldsymbol{x}}_{I}\right)=\sum_{N=1}^{\infty}(-1)^{N} c^{(N)}\left(\boldsymbol{v}, \overrightarrow{\boldsymbol{x}}_{I}\right)$, with $c\left(\boldsymbol{v}, \overrightarrow{\boldsymbol{x}}_{I}\right)=0$ when $|I|=1$. Thus $B\left(\boldsymbol{v}, \overrightarrow{\boldsymbol{x}}_{I}\right)=b\left(\boldsymbol{v}, \overrightarrow{\boldsymbol{x}}_{I}\right)+c\left(\boldsymbol{v}, \overrightarrow{\boldsymbol{x}}_{I}\right)$ with

$$
b\left(\boldsymbol{v}, \overrightarrow{\boldsymbol{x}}_{I}\right)=\sum_{N=0}^{\infty}(-1)^{N} b^{(N)}\left(\boldsymbol{v}, \overrightarrow{\boldsymbol{x}}_{I}\right),
$$

where $b^{(0)}\left(\boldsymbol{v}, \overrightarrow{\boldsymbol{x}}_{I}\right)=B^{(0)}\left(\boldsymbol{v}, \overrightarrow{\boldsymbol{x}}_{I}\right)$ and

$$
\begin{aligned}
b^{(N)}\left(\boldsymbol{v}, \overrightarrow{\boldsymbol{x}}_{I}\right)= & \sum_{j=0}^{N} \sum_{\left(\boldsymbol{u}_{0}, \boldsymbol{v}_{0}\right)} \ldots \sum_{\left(\boldsymbol{u}_{N}, \boldsymbol{v}_{N}\right)} \delta_{\boldsymbol{v}_{N}, \boldsymbol{v}}\left[\prod_{i=0}^{N} p D\left(\boldsymbol{v}_{i}-\boldsymbol{u}_{i}\right)\right] \mathbb{E}_{0} I\left[\mathbf{0} \Rightarrow \boldsymbol{u}_{0}\right] \\
& \times \mathbb{E}_{1} I\left[E^{\prime}\left(\boldsymbol{v}_{0}, \boldsymbol{u}_{1} ; \widetilde{\boldsymbol{C}}_{0}\right)\right] \ldots \mathbb{E}_{j-1} I\left[E^{\prime}\left(\boldsymbol{v}_{j-2}, \boldsymbol{u}_{j-1} ; \widetilde{C}_{j-2}\right)\right] \\
& \times \mathbb{E}_{j} I\left[G^{\prime \prime}\left(\boldsymbol{v}_{j-1}, \boldsymbol{u}_{j}, \boldsymbol{v}_{j}, \overrightarrow{\boldsymbol{x}}_{I} ; \widetilde{C}_{j-1}\right)\right] \\
& \times \mathbb{E}_{j+1} I\left[E^{\prime}\left(\boldsymbol{v}_{j}, \boldsymbol{u}_{j+1} ; \widetilde{C}_{j}\right)\right] \ldots \mathbb{E}_{N} I\left[E^{\prime}\left(\boldsymbol{v}_{N-1}, \boldsymbol{u}_{N} ; \widetilde{C}_{N-1}\right)\right] \quad(N \geqslant 1) .
\end{aligned}
$$

Here, we have used (5.17) to replace $G^{\prime \prime}$ by $E^{\prime \prime}$ in all expectations other than $\mathbb{E}_{j}$, and then replaced $E^{\prime \prime}$ by $E^{\prime}$ as discussed below (3.21). The form of (6.3) is slightly different when $j=0$, in which case $E^{\prime}$ events appear in all expectations except $\mathbb{E}_{0}$, where the 
event $F^{\prime \prime}\left(\boldsymbol{u}_{0}, \boldsymbol{v}_{0}, \overrightarrow{\boldsymbol{x}}_{I}\right)$ appears as in (5.26). Note that the form of $b^{(N)}\left(\boldsymbol{v}, \overrightarrow{\boldsymbol{x}}_{I}\right)$ is the same as that of $\left(\pi^{(N)} * p D\right)(\boldsymbol{v})$ (see (3.25)), apart from the event occurring at level- $j$.

The goal of the second expansion is to "cut off" the connection to $\overrightarrow{\boldsymbol{x}}_{I}$ in the configuration at level- $j$, to produce an identity

$$
b\left(\boldsymbol{v}, \overrightarrow{\boldsymbol{x}}_{I}\right)=\sum_{\boldsymbol{w}} C(\boldsymbol{v}, \boldsymbol{w}) \tau^{(|I|+1)}\left(\overrightarrow{\boldsymbol{x}}_{I}-\boldsymbol{w}\right)+d\left(\boldsymbol{v}, \overrightarrow{\boldsymbol{x}}_{I}\right) .
$$

Eq. (6.4) will give the desired result (6.1), with

$$
R\left(\boldsymbol{v}, \overrightarrow{\boldsymbol{x}}_{I}\right)=c\left(\boldsymbol{v}, \overrightarrow{\boldsymbol{x}}_{I}\right)+d\left(\boldsymbol{v}, \overrightarrow{\boldsymbol{x}}_{I}\right) .
$$

The derivation of (6.4) will require a detailed study of the nature of the level- $j$ configuration. The level- $j$ configuration enters (6.3) both at level- $j$, where the configuration is required to exhibit the connections inherent in the definition of the event $G^{\prime \prime}\left(\boldsymbol{v}_{j-1}, \boldsymbol{u}_{j}, \boldsymbol{v}_{j}, \overrightarrow{\boldsymbol{x}}_{I} ; \widetilde{C}_{j-1}\right)$, and at level- $(j+1)($ when $j \neq N)$, where the level- $j$ cluster $\widetilde{C}_{j}$ is required to intersect the level- $(j+1)$ configuration in the manner prescribed by the event $E^{\prime}\left(\boldsymbol{v}_{j}, \boldsymbol{u}_{j+1} ; \widetilde{C}_{j}\right)$. We will focus in what follows on the generic case in which $0<j<N$, as the modifications required for the special cases $j=0$ and $j=N$ are routine.

In order to fix the cluster at level- $(j+1)$, so that all demands on the level- $j$ configuration can be clearly specified, we will use Fubini's Theorem to interchange the expectations $\mathbb{E}_{j}$ and $\mathbb{E}_{j+1}$. Let

$$
H_{j}\left(\overrightarrow{\boldsymbol{x}}_{I}\right)=G^{\prime \prime}\left(\boldsymbol{v}_{j-1}, \boldsymbol{u}_{j}, \boldsymbol{v}_{j}, \overrightarrow{\boldsymbol{x}}_{I} ; \widetilde{C}_{j-1}\right) \cap E^{\prime}\left(\boldsymbol{v}_{j}, \boldsymbol{u}_{j+1} ; \widetilde{C}_{j}\right),
$$

where we suppress the dependence of $H_{j}$ on the $\boldsymbol{u}_{i}, \boldsymbol{v}_{i}$ from the notation. (For $j=N$ the event $E^{\prime}$ should be omitted in (6.6), and for $j=0$ the event $G^{\prime \prime}$ should be replaced by $\left.F^{\prime \prime}.\right)$ In (6.6), the event $E^{\prime}\left(\boldsymbol{v}_{j}, \boldsymbol{u}_{j+1} ; \widetilde{C}_{j}\right)$ is regarded as a restriction on the level$j$ configuration, given the level- $(j+1)$ configuration. It then follows from Fubini's Theorem that

$$
\begin{aligned}
b^{(N)}\left(\boldsymbol{v}, \overrightarrow{\boldsymbol{x}}_{I}\right)= & \sum_{j=0}^{N} \sum_{\left(\boldsymbol{u}_{0}, \boldsymbol{v}_{0}\right)} \ldots \sum_{\left(\boldsymbol{u}_{N}, \boldsymbol{v}_{N}\right)} \delta_{\boldsymbol{v}_{N}, \boldsymbol{v}}\left[\prod_{i=0}^{N} p D\left(\boldsymbol{v}_{i}-\boldsymbol{u}_{i}\right)\right] \mathbb{E}_{0} I\left[\mathbf{0} \Rightarrow \boldsymbol{u}_{0}\right] \\
& \times \mathbb{E}_{1} I\left[E^{\prime}\left(\boldsymbol{v}_{0}, \boldsymbol{u}_{1} ; \widetilde{C}_{0}\right)\right] \ldots \mathbb{E}_{j-1} I\left[E^{\prime}\left(\boldsymbol{v}_{j-2}, \boldsymbol{u}_{j-1} ; \widetilde{C}_{j-2}\right)\right] \\
& \times \mathbb{E}_{j+1} \mathbb{P}_{j}\left(H_{j}\left(\overrightarrow{\boldsymbol{x}}_{I}\right)\right) \mathbb{E}_{j+2} I\left[E^{\prime}\left(\boldsymbol{v}_{j+1}, \boldsymbol{u}_{j+2} ; \widetilde{C}_{j+1}\right)\right] \ldots \\
& \times \mathbb{E}_{N} I\left[E^{\prime}\left(\boldsymbol{v}_{N-1}, \boldsymbol{u}_{N} ; \widetilde{C}_{N-1}\right)\right] .
\end{aligned}
$$

The second expansion will take place within $\mathbb{P}_{j}\left(H_{j}\left(\overrightarrow{\boldsymbol{x}}_{I}\right)\right)$.

To carry out the second expansion, we will need to define a "cutting bond" as in Sections 3 and 5. For this, we begin by decomposing the event $G^{\prime}\left(\boldsymbol{v}_{j-1}, \boldsymbol{u}_{j}, \overrightarrow{\boldsymbol{x}}_{I} ; \widetilde{\boldsymbol{C}}_{j-1}\right)$ that is relevant for $H_{j}\left(\overrightarrow{\boldsymbol{x}}_{I}\right)$ (see (5.15)). We define

$$
\begin{aligned}
& G_{1}^{\prime}\left(\boldsymbol{v}_{j-1}, \boldsymbol{u}_{j}, \overrightarrow{\boldsymbol{x}}_{I} ; \widetilde{C}_{j-1}\right)=G^{\prime}\left(\boldsymbol{v}_{j-1}, \boldsymbol{u}_{j}, \overrightarrow{\boldsymbol{x}}_{I} ; \widetilde{C}_{j-1}\right) \cap E^{\prime}\left(\boldsymbol{v}_{j-1}, \boldsymbol{u}_{j} ; \widetilde{C}_{j-1}\right), \\
& G_{2}^{\prime}\left(\boldsymbol{v}_{j-1}, \boldsymbol{u}_{j}, \overrightarrow{\boldsymbol{x}}_{I} ; \widetilde{C}_{j-1}\right)=G^{\prime}\left(\boldsymbol{v}_{j-1}, \boldsymbol{u}_{j}, \overrightarrow{\boldsymbol{x}}_{I} ; \widetilde{C}_{j-1}\right) \cap\left(E^{\prime}\left(\boldsymbol{v}_{j-1}, \boldsymbol{u}_{j} ; \widetilde{C}_{j-1}\right)\right)^{c},
\end{aligned}
$$


so that $G^{\prime}\left(\boldsymbol{v}_{j-1}, \boldsymbol{u}_{j}, \overrightarrow{\boldsymbol{x}}_{I} ; \widetilde{C}_{j-1}\right)$ is the disjoint union of the two events $G_{1}^{\prime}\left(\boldsymbol{v}_{j-1}, \boldsymbol{u}_{j}, \overrightarrow{\boldsymbol{x}}_{I}\right.$; $\left.\widetilde{C}_{j-1}\right)$ and $G_{2}^{\prime}\left(\boldsymbol{v}_{j-1}, \boldsymbol{u}_{j}, \overrightarrow{\boldsymbol{x}}_{I} ; \widetilde{C}_{j-1}\right)$. The events $G_{1}^{\prime}$ and $G_{2}^{\prime}$ should be compared respectively to the cases (i) and (ii) above (5.15). As usual, we use $G_{i}^{\prime \prime}$ to denote the event that $G_{i}^{\prime}$ occurs on $\widetilde{C}_{j}$. We also define $\widetilde{C}_{j}^{(a, b)}$ to be the vertices of $\widetilde{C}_{j}$ that remain connected to $\boldsymbol{v}_{j-1}$ after $(\boldsymbol{a}, \boldsymbol{b})$ is made vacant. The cutting bond is defined differently on $G_{1}^{\prime \prime}$ and $G_{2}^{\prime \prime}$, as follows: (i) Given a configuration on which $H_{j}\left(\overrightarrow{\boldsymbol{x}}_{I}\right) \cap G_{1}^{\prime \prime}$ occurs, the cutting bond $(\boldsymbol{a}, \boldsymbol{b})$ is defined to be the first pivotal bond (if it exists) for the event $\left\{\boldsymbol{v}_{j-1} \rightarrow \boldsymbol{x}_{i} \forall i \in I\right\}$ such that $E^{\prime}\left(\boldsymbol{v}_{j-1}, \boldsymbol{u}_{j} ; \widetilde{C}_{j-1}\right) \cap E^{\prime}\left(\boldsymbol{v}_{j}, \boldsymbol{u}_{j+1} ; \widetilde{C}_{j}\right)$ occurs on $\widetilde{C}_{j}^{(a, b)}$. (ii) Given a configuration on which $H_{j}\left(\overrightarrow{\boldsymbol{x}}_{I}\right) \cap G_{2}^{\prime \prime}$ occurs, the cutting bond $(\boldsymbol{a}, \boldsymbol{b})$ is defined to be the first pivotal bond (if it exists) for the event $\left\{\boldsymbol{v}_{j-1} \rightarrow \boldsymbol{x}_{i} \forall i \in I\right\}$ such that there exists a $\boldsymbol{y}$ such that

$$
\left\{\boldsymbol{v}_{j-1} \rightarrow \boldsymbol{y} \stackrel{\widetilde{C}_{j-1}}{\longrightarrow} \boldsymbol{a}\right\} \cap\left\{\boldsymbol{y} \Rightarrow \boldsymbol{u}_{j}\right\} \cap E^{\prime}\left(\boldsymbol{v}_{j}, \boldsymbol{u}_{j+1} ; \widetilde{C}_{j}\right)
$$

occurs on $\widetilde{C}_{j}^{(a, b)}$. Examples of cutting bonds are depicted in Fig. 10.

The event $H_{j}$ can be partitioned according to the location of the cutting bond, or the absence of a cutting bond. For this, we define

$$
\begin{aligned}
H_{j}^{\prime}\left(\overrightarrow{\boldsymbol{x}}_{I}\right) & =H_{j}\left(\overrightarrow{\boldsymbol{x}}_{I}\right) \cap\left\{\nexists \text { a cutting bond for } \boldsymbol{v}_{j-1} \rightarrow \overrightarrow{\boldsymbol{x}}_{I}\right\}, \\
H_{j}^{\prime \prime}(\boldsymbol{a}, \boldsymbol{b}) & =\left\{H_{j}^{\prime}(\boldsymbol{a}) \text { occurs on } \widetilde{C}_{j}^{(\boldsymbol{a}, \boldsymbol{b})}\right\} .
\end{aligned}
$$

In view of the pivotal nature of the cutting bond $(\boldsymbol{a}, \boldsymbol{b})$, as in (5.9) we have
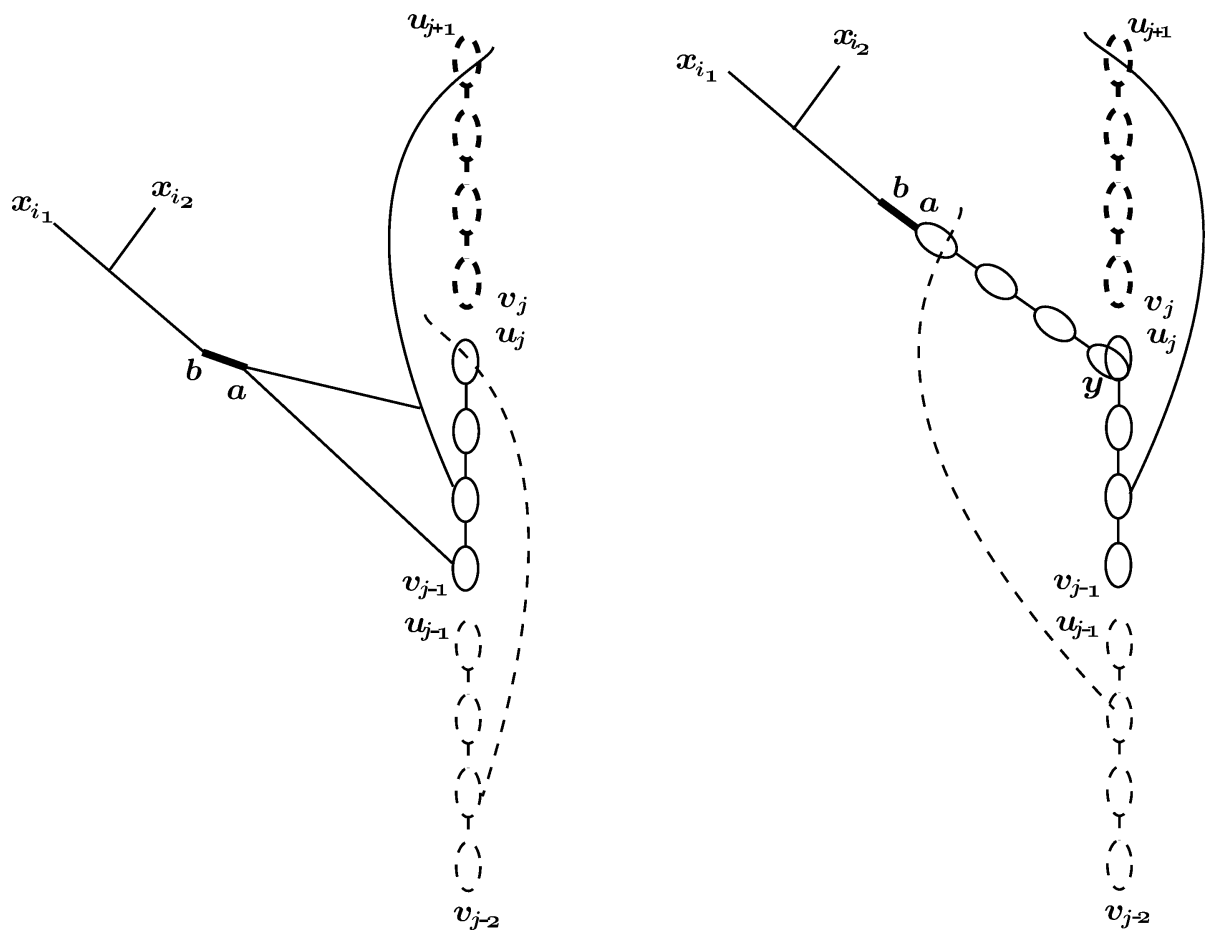

Fig. 10. Examples of cutting bonds ( $\boldsymbol{a}, \boldsymbol{b})$ for the second expansion, for $G_{1}^{\prime \prime}$ (left) and $G_{2}^{\prime \prime}$ (right). 


$$
\begin{gathered}
H_{j}\left(\overrightarrow{\boldsymbol{x}}_{I}\right)=H_{j}^{\prime}\left(\overrightarrow{\boldsymbol{x}}_{I}\right) \bigcup_{(\boldsymbol{a}, \boldsymbol{b})}\left(H_{j}^{\prime \prime}(\boldsymbol{a}, \boldsymbol{b}) \cap\{(\boldsymbol{a}, \boldsymbol{b}) \text { occupied }\}\right. \\
\left.\cap\left\{F\left(\boldsymbol{b}, \overrightarrow{\boldsymbol{x}}_{I}\right) \text { occurs in } \Lambda \backslash \widetilde{\boldsymbol{C}}_{j}^{(\boldsymbol{a}, \boldsymbol{b})}\right\}\right) .
\end{gathered}
$$

Using Lemma 3.3 (now applied to the lattice with the bond $(\boldsymbol{u}, \boldsymbol{v})$ removed) and $(5.12)$ gives

$$
\begin{aligned}
\mathbb{P}_{j}\left(H_{j}\left(\overrightarrow{\boldsymbol{x}}_{I}\right)\right)= & \mathbb{P}_{j}\left(H_{j}^{\prime}\left(\overrightarrow{\boldsymbol{x}}_{I}\right)\right)+\sum_{(\boldsymbol{a}, \boldsymbol{b})} p D(\boldsymbol{b}-\boldsymbol{a}) \mathbb{E}_{j, 0}\left(I\left[H_{j}^{\prime \prime}(\boldsymbol{a}, \boldsymbol{b})\right] \tau^{\widetilde{C}_{j, 0}^{(a, b)}}\left(\boldsymbol{b}, \overrightarrow{\boldsymbol{x}}_{I}\right)\right) \\
= & \mathbb{P}_{j}\left(H_{j}^{\prime}\left(\overrightarrow{\boldsymbol{x}}_{I}\right)\right)+\sum_{(\boldsymbol{a}, \boldsymbol{b})} p D(\boldsymbol{b}-\boldsymbol{a}) \mathbb{P}_{j, 0}\left(H_{j}^{\prime \prime}(\boldsymbol{a}, \boldsymbol{b})\right) \tau^{(I+1)}\left(\overrightarrow{\boldsymbol{x}}_{I}-\boldsymbol{b}\right) \\
& -\sum_{(\boldsymbol{a}, \boldsymbol{b})} p D(\boldsymbol{b}-\boldsymbol{a}) \mathbb{E}_{j, 0}\left(I\left[H_{j}^{\prime \prime}(\boldsymbol{a}, \boldsymbol{b})\right] \mathbb{P}_{j, 1}\left(\boldsymbol{b} \stackrel{\widetilde{C}_{j, 0}^{(\boldsymbol{a}, \boldsymbol{b})}}{\longrightarrow} \overrightarrow{\boldsymbol{x}}_{I}\right)\right)
\end{aligned}
$$

The subscripts $j, 0$ on $\widetilde{C}_{j, 0}^{(a, b)}$ denote level- 0 of the second expansion applied to level- $j$ of the first expansion.

The expansion could be continued by repeatedly using the recursion (5.21) for the probability $\mathbb{P}_{j, 1}\left(\boldsymbol{b} \stackrel{\widetilde{C}_{j, 0}^{(a, b)}}{\longrightarrow} \overrightarrow{\boldsymbol{x}}_{I}\right)$ in (6.13). However, we can now use a somewhat simpler organisation, which effectively stops the expansion earlier. Thus, given a configuration in which $\boldsymbol{b} \stackrel{A}{\rightarrow} \overrightarrow{\boldsymbol{x}}_{I}$, we will now define the cutting bond to be the first bond $\left(\boldsymbol{a}^{\prime}, \boldsymbol{b}^{\prime}\right)$ (in the direction $\left.\boldsymbol{b} \rightarrow \overrightarrow{\boldsymbol{x}}_{I}\right)$ such that (i) $\left(\boldsymbol{a}^{\prime}, \boldsymbol{b}^{\prime}\right)$ is pivotal for $\boldsymbol{b} \rightarrow \boldsymbol{x}_{i}$ for all $i \in I$, and (ii) $\boldsymbol{b} \stackrel{A}{\rightarrow} \boldsymbol{a}^{\prime}$. It is possible that no such bond exists. Let

$$
E^{\prime}\left(\boldsymbol{b}, \overrightarrow{\boldsymbol{x}}_{I} ; A\right)=\left\{\boldsymbol{b} \stackrel{A}{\rightarrow} \overrightarrow{\boldsymbol{x}}_{I}\right\} \cap\{\nexists \text { cutting bond }\}
$$

and recall the definition $E^{\prime \prime}\left(\boldsymbol{b}, \boldsymbol{a}^{\prime}, \boldsymbol{b}^{\prime} ; A\right)=\left\{E^{\prime}\left(\boldsymbol{b}, \boldsymbol{a}^{\prime} ; A\right)\right.$ occurs on $\left.\widetilde{C}^{\left(\boldsymbol{a}^{\prime}, \boldsymbol{b}^{\prime}\right)}(\boldsymbol{b})\right\}$ from (3.19). Then, as in (5.9), we have

$$
\begin{aligned}
\left\{\boldsymbol{b} \stackrel{A}{\rightarrow} \overrightarrow{\boldsymbol{x}}_{I}\right\}= & E^{\prime}\left(\boldsymbol{b}, \overrightarrow{\boldsymbol{x}}_{I} ; A\right) \bigcup_{\left(\boldsymbol{a}^{\prime}, \boldsymbol{b}^{\prime}\right)}\left(E^{\prime \prime}\left(\boldsymbol{b}, \boldsymbol{a}^{\prime}, \boldsymbol{b}^{\prime} ; A\right) \cap\left\{\left(\boldsymbol{a}^{\prime}, \boldsymbol{b}^{\prime}\right) \text { occupied }\right\}\right. \\
& \left.\cap\left\{F\left(\boldsymbol{b}^{\prime}, \overrightarrow{\boldsymbol{x}}_{I}\right) \text { occurs in } \Lambda \backslash \widetilde{\boldsymbol{C}}^{\left(\boldsymbol{a}^{\prime}, \boldsymbol{b}^{\prime}\right)}(\boldsymbol{b})\right\}\right) .
\end{aligned}
$$

As usual, using Lemma 3.3, this leads to

$$
\begin{aligned}
\mathbb{P}_{j, 1}\left(\boldsymbol{b} \stackrel{A}{\rightarrow} \overrightarrow{\boldsymbol{x}}_{I}\right)= & \mathbb{P}_{j, 1}\left(E^{\prime}\left(\boldsymbol{b}, \overrightarrow{\boldsymbol{x}}_{I} ; A\right)\right)+\sum_{\left(\boldsymbol{a}^{\prime}, \boldsymbol{b}^{\prime}\right)} p D\left(\boldsymbol{b}^{\prime}-\boldsymbol{a}^{\prime}\right) \mathbb{P}_{j, 1}\left(E^{\prime}\left(\boldsymbol{b}, \boldsymbol{a}^{\prime} ; A\right)\right) \tau^{(|I|+1)}\left(\overrightarrow{\boldsymbol{x}}_{I}-\boldsymbol{b}^{\prime}\right) \\
& -\sum_{\left(\boldsymbol{a}^{\prime}, \boldsymbol{b}^{\prime}\right)} p D\left(\boldsymbol{b}^{\prime}-\boldsymbol{a}^{\prime}\right) \mathbb{E}_{j, 1}\left(I\left[E^{\prime}(\boldsymbol{b}, \boldsymbol{a} ; A)\right] \mathbb{P}_{j, 2}\left(\boldsymbol{b}^{\prime} \stackrel{\widetilde{C}_{j, 1}^{\left(\boldsymbol{a}^{\prime}, \boldsymbol{b}^{\prime}\right)}(\boldsymbol{b})}{\longrightarrow} \overrightarrow{\boldsymbol{x}}_{I}\right)\right)
\end{aligned}
$$

where we have replaced the $E^{\prime \prime}$ events by $E^{\prime}$ events as explained below (3.21).

The second expansion is generated by recursively using (6.16) in (6.13), and substituting the result in (6.7). The result is (6.4), with $C$ and $d$ of the form 


$$
\begin{aligned}
C(\boldsymbol{v}, \boldsymbol{w}) & =\sum_{M, N=0}^{\infty}(-1)^{N+M} C^{(N, M)}(\boldsymbol{v}, \boldsymbol{w}), \\
d\left(\boldsymbol{v}, \overrightarrow{\boldsymbol{x}}_{I}\right) & =\sum_{M, N=0}^{\infty}(-1)^{N+M} d^{(N, M)}\left(\boldsymbol{v}, \overrightarrow{\boldsymbol{x}}_{I}\right) .
\end{aligned}
$$

Here $d\left(\boldsymbol{v}, \overrightarrow{\boldsymbol{x}}_{I}\right)$ arises from the $\mathbb{P}_{j}\left(H_{j}^{\prime}\left(\overrightarrow{\boldsymbol{x}}_{I}\right)\right)$ term in (6.13) and from the term $\mathbb{P}\left(E^{\prime}\left(\boldsymbol{b}, \overrightarrow{\boldsymbol{x}}_{I}\right.\right.$; $A))$ in $(6.16)$ or its iterates. The term $C^{(N, M)}(\boldsymbol{v}, \boldsymbol{w})$ is given by (6.7) with $\mathbb{P}_{j}\left(H_{j}\left(\overrightarrow{\boldsymbol{x}}_{I}\right)\right)$ replaced by the expression $\sum_{\boldsymbol{a}} \mathbb{P}_{j, 0}\left(H_{j}^{\prime \prime}(\boldsymbol{a}, \boldsymbol{w})\right) p D(\boldsymbol{w}-\boldsymbol{a})$ for $M=0$, and by

$$
\begin{aligned}
& \sum_{\left(\boldsymbol{a}_{0}, \boldsymbol{b}_{0}\right)} \ldots \sum_{\left(\boldsymbol{a}_{M}, \boldsymbol{b}_{M}\right)} \delta_{\boldsymbol{b}_{M}, \boldsymbol{w}}\left[\prod_{i=0}^{M} p D\left(\boldsymbol{b}_{i}-\boldsymbol{a}_{i}\right)\right] \mathbb{E}_{j, 0} I\left[H_{j}^{\prime \prime}\left(\boldsymbol{a}_{0}, \boldsymbol{b}_{0}\right)\right] \\
& \quad \times \mathbb{E}_{j, 1} I\left[E^{\prime}\left(\boldsymbol{b}_{0}, \boldsymbol{a}_{1} ; \widetilde{C}_{j, 0}\right)\right] \ldots \mathbb{E}_{j, M} I\left[E^{\prime}\left(\boldsymbol{b}_{M-1}, \boldsymbol{a}_{M} ; \widetilde{C}_{j, M-1}\right)\right]
\end{aligned}
$$

for $M \geqslant 1$. The term $d^{(N, M)}\left(\boldsymbol{v}, \overrightarrow{\boldsymbol{x}}_{I}\right)$ is given by (6.7) with $\mathbb{P}_{j}\left(H_{j}\left(\overrightarrow{\boldsymbol{x}}_{I}\right)\right)$ replaced by $\mathbb{P}_{j}\left(H_{j}^{\prime}\left(\overrightarrow{\boldsymbol{x}}_{I}\right)\right)$ for $M=0$, and by

$$
\begin{aligned}
& \sum_{\left(\boldsymbol{a}_{0}, \boldsymbol{b}_{0}\right)} \ldots \sum_{\left(\boldsymbol{a}_{M-1}, \boldsymbol{b}_{M-1}\right)}\left[\prod_{i=0}^{M-1} p D\left(\boldsymbol{b}_{i}-\boldsymbol{a}_{i}\right)\right] \mathbb{E}_{j, 0} I\left[H_{j}^{\prime \prime}\left(\boldsymbol{a}_{0}, \boldsymbol{b}_{0}\right)\right] \\
& \times \mathbb{E}_{j, 1} I\left[E^{\prime}\left(\boldsymbol{b}_{0}, \boldsymbol{a}_{1} ; \widetilde{C}_{j, 0}\right)\right] \ldots \mathbb{E}_{j, M} I\left[E^{\prime}\left(\boldsymbol{b}_{M-1}, \overrightarrow{\boldsymbol{x}}_{I} ; \widetilde{C}_{j, M-1}\right)\right]
\end{aligned}
$$

for $M \geqslant 1$.

\section{Proof of Proposition 2.3}

In this section, we prove Proposition 2.3, which completes the proof of Theorem 1.2. In Section 7.1, we reduce the proof of Proposition 2.3 to Lemma 7.1, which provides estimates on quantities arising in the expansions of Sections 5 and 6. In Section 7.2, we show how these quantities can be bounded in terms of diagrams using the BK inequality, as was done for $\pi_{n}(x)$ in Section 4.1. Finally, in Section 7.3, we estimate the diagrams and prove Lemma 7.1.

\subsection{The main estimates}

Proposition 2.3 gives bounds on the Fourier transforms of $\psi_{m_{1}, m_{2}}\left(y_{1}, y_{2}\right)$ and $\varphi_{\vec{n}}^{(r)}(\vec{x})$, and we begin by recalling their definitions. As usual, we write $\vec{n}=\left(n_{1}, \ldots, n_{r-1}\right), \underline{n}=$ $\min \left\{n_{1}, \ldots, n_{r-1}\right\}, J=\{1, \ldots, r\}, J_{1}=J \backslash\{1\}$, and given $I \subset J_{1}$, we let $r_{1}=|J \backslash I|+1$ and $r_{2}=|I|+1$. Also, for any $I \subset J$, we write $\vec{k}_{I}=\left(k_{i}\right)_{i \in I}$ and $k_{I}=\sum_{i \in I} k_{i}$. In this section, we will use the $(x, n)$ notation for lattice sites, rather than $\boldsymbol{x}$.

The function $\psi$ is given in (2.42) by

$$
\psi_{m_{1}, m_{2}}\left(y_{1}, y_{2}\right)=\sum_{u \in \mathbb{Z}^{d}} p_{c} D(u) C_{m_{1}-1, m_{2}-1}\left(y_{1}-u, y_{2}-u\right),
$$


and $\varphi^{(r)}$ is given in (2.43) by

$$
\varphi_{\vec{n}}^{(r)}(\vec{x})=\alpha_{\vec{n}}^{(r)}(\vec{x})+\sum_{v \in \mathbb{Z}^{d}} \sum_{j=1}^{\underline{n}} p_{c}\left(\tau_{j-1} * D\right)(v) g_{\vec{n}-j}^{(r)}(\vec{x}-v) .
$$

The quantities $\alpha_{\vec{n}}^{(r)}(\vec{x})$ and $g_{\vec{n}}^{(r)}(\vec{x})$ are given in (2.33) and (2.41) by

$$
\begin{aligned}
& \alpha_{\vec{n}}^{(r)}(\vec{x})=A_{\vec{n}}^{(r)}(\vec{x})+\sum_{I \subset J_{1}: I \neq \emptyset} \sum_{v} \sum_{j=1}^{n_{J \backslash I}} B_{j, \vec{n}_{I}}^{\left(r_{2}+1\right)}\left(v, \vec{x}_{I}\right) \tau_{\vec{n}_{J \backslash I}^{\left(r_{1}\right)}-j}\left(\vec{x}_{J \backslash I}-v\right), \\
& g_{\vec{n}}^{(r)}(\vec{x})=A_{\vec{n}}^{(r)}(\vec{x})+\sum_{I \subset J_{1}: I \neq \emptyset} \sum_{v} \sum_{l=1}^{\underline{n}_{J \backslash I}} R_{l, \vec{n}_{I}}^{\left(r_{2}+1\right)}\left(v, \vec{x}_{I}\right) \tau_{\vec{n}_{J \backslash I}^{\left(r_{1}\right)}-l}\left(\vec{x}_{J \backslash I}-v\right),
\end{aligned}
$$

with $R^{\left(r_{2}+1\right)}$ and $B^{\left(r_{2}+1\right)}$ given by (6.5), and above (6.2), as

$$
\begin{aligned}
& R_{j, \vec{n}_{I}}^{\left(r_{2}+1\right)}\left(v, \vec{x}_{I}\right)=c_{j, \vec{n}_{I}}^{\left(r_{2}+1\right)}\left(v, \vec{x}_{I}\right)+d_{j, \vec{n}_{I}}^{\left(r_{2}+1\right)}\left(v, \vec{x}_{I}\right), \\
& B_{j, \vec{n}_{I}}^{\left(r_{2}+1\right)}\left(v, \vec{x}_{I}\right)=b_{j, \vec{n}_{I}}^{\left(r_{2}+1\right)}\left(v, \vec{x}_{I}\right)+c_{j, \vec{n}_{I}}^{\left(r_{2}+1\right)}\left(v, \vec{x}_{I}\right) .
\end{aligned}
$$

The following lemma, whose proof is deferred to Section 7.3, provides estimates sufficient to prove Proposition 2.3.

Lemma 7.1. - Let $d>4, p=p_{c}, s \geqslant 3, \vec{n}=\left(n_{1}, \ldots, n_{s-1}\right)$ with each $n_{i}>0$, and let $\vec{n}$ denote the second largest component of $\vec{n}$. There exists an $L_{0}=L_{0}(d)$ and a constant $C=C(d, s)$ such that for all $\vec{k} \in[-\pi, \pi]^{s-1}, q \in\{0,2\}, L \geqslant L_{0}$,

$$
\begin{aligned}
& \left|\hat{A}_{\vec{n}}^{(s)}(\vec{k})\right| \leqslant C \bar{n}^{s-3}\left(n_{1}+1\right)^{-(d-2) / 2}+C \bar{n}^{s-4}\left(n_{1}+1\right)^{-(d-4) / 2}, \\
& \left|\hat{b}_{\vec{n}}^{(s)}(\vec{k})\right| \leqslant C \bar{n}^{s-3}\left(n_{1}+1\right)^{-(d-2) / 2}, \\
& \left|\hat{c}_{\vec{n}}^{(s)}(\vec{k})\right| \leqslant C \bar{n}^{s-4}\left(n_{1}+1\right)^{-(d-4) / 2} \quad(s \geqslant 4), \\
& \left|\hat{d}_{\vec{n}}^{(s)}(\vec{k})\right| \leqslant \begin{cases}C\left(n_{1} \vee n_{2}+1\right)^{-d / 2} & (s=3), \\
C \bar{n}^{s-4}\left(n_{1}+1\right)^{-(d-4) / 2} & (s \geqslant 4),\end{cases} \\
& \sum_{x_{1}, x_{2}}\left|x_{i}\right|^{q}\left|C_{n_{1}, n_{2}}\left(x_{1}, x_{2}\right)\right| \\
& \leqslant C \beta \sigma^{q} n_{i}^{q / 2}\left(n_{1} \vee n_{2}+1\right)^{-d / 2} \quad\left(\left(n_{1}, n_{2}\right) \neq(1,1)\right) .
\end{aligned}
$$

Proof of Proposition 2.3 assuming Lemma 7.1. - (i) For $\left(m_{1}, m_{2}\right)=(2,2)$, it follows from $(2.45)$ that $\hat{\psi}_{2,2}\left(k_{1}, k_{2}\right)=p_{c}^{3} \widehat{D}\left(k_{1}+k_{2}\right) \widehat{D}\left(k_{1}\right) \widehat{D}\left(k_{2}\right)$, which obeys the bound (2.47) of Proposition 2.3(i). The desired bound for $\left(m_{1}, m_{2}\right) \neq(2,2)$ follows immediately from (7.11) and (7.1) (including the factor $\beta$ mentioned at the end of Proposition 2.3).

In addition, $\nabla_{i} \hat{\psi}_{m_{1}, m_{2}}(0,0)=\sum_{x_{1}, x_{2}} x_{i} \psi_{m_{1}, m_{2}}\left(x_{1}, x_{2}\right)=0$, because it is a consequence of the lattice symmetry that $\sum_{x_{1}} C_{m_{1}, m_{2}}\left(x_{1}, x_{2}\right)=\sum_{x_{1}} C_{m_{1}, m_{2}}\left(x_{1},-x_{2}\right)$ and $\sum_{x_{2}} C_{m_{1}, m_{2}}\left(x_{1}, x_{2}\right)=\sum_{x_{2}} C_{m_{1}, m_{2}}\left(-x_{1}, x_{2}\right)$.

(ii) Our goal is to prove that

$$
\left|\hat{\varphi}_{\vec{n}}^{(r)}(\vec{k})\right| \leqslant C \bar{n}^{r-2-\delta} \quad(r \geqslant 3)
$$


where $\delta$ is fixed in $\left(0,1 \wedge \varepsilon \wedge \frac{d-4}{2}\right)$. By the tree-graph bound [3, Proposition 4.1] and the uniform bound on $\hat{\tau}_{n}(k)$ provided by Theorem 1.1(a),

$$
\left|\hat{\tau}_{\vec{n}}^{(r)}(\vec{k})\right| \leqslant C \bar{n}^{r-2} \quad(r \geqslant 2) .
$$

In (7.13), the factor $\bar{n}^{r-2}$ can be understood from the fact that each of the $r-2$ temporal summations in the tree-graph bound stop at most at $\bar{n}$, since these sums correspond to the temporal components of branch points in the tree graph bound and these are at most $\bar{n}$. In conjunction with (7.2)-(7.4), it follows from (7.13) that

$$
\begin{aligned}
\left|\hat{\varphi}_{\vec{n}}^{(r)}(\vec{k})\right| \leqslant & C \sum_{j=0}^{\underline{n}}\left|\hat{A}_{\vec{n}-j}^{(r)}(\vec{k})\right|+C \sum_{I \subset J_{1}: I \neq \emptyset} \sum_{j=0}^{\underline{n}} \bar{n}^{r_{1}-2}\left[\left|\hat{B}_{j, \vec{n}_{I}-1}^{\left(r_{2}+1\right)}\left(k_{J \backslash I}, \vec{k}_{I}\right)\right|\right. \\
& \left.+\sum_{l=1}^{\underline{n}_{J \backslash I}-j}\left|\hat{R}_{l, \vec{n}_{I}-j}^{\left(r_{2}+1\right)}\left(k_{J \backslash I}, \vec{k}_{I}\right)\right|\right] .
\end{aligned}
$$

The contribution to the first term on the right side of (7.14) due to the first term of (7.7) is bounded by

$$
C \bar{n}^{r-3} \sum_{j=0}^{\underline{n}}\left(n_{1}-j+1\right)^{-(d-2) / 2} \leqslant C \bar{n}^{r-3},
$$

as required. Since the choice of $\boldsymbol{x}_{1}$ as the direction in which to perform the expansion was arbitrary, we may assume without loss of generality that $n_{1}$ is not the largest component of $\vec{n}$, so that $n_{1} \leqslant \bar{n}$. In this case, the second term of (7.7) is bounded above by the first term of (7.7), and hence also leads to the bound (7.15).

For the remaining terms, note that for each nonempty $I$ we have $r_{1}+r_{2}=r+1$ and $r_{2}+1=|I|+2 \geqslant 3$. To bound the term on the right side of (7.14) containing $B$, we recall that $c^{(3)}=0$ by definition, use (7.8)-(7.9), and note that $\bar{n}^{s-4}(j+1)^{-(d-4) / 2} \leqslant$ $\bar{n}^{s-3}(j+1)^{-(d-2) / 2}$ since $j \leqslant \underline{n} \leqslant \bar{n}$. The term in (7.14) containing $B$ is therefore bounded above by

$$
C \sum_{I \subset J_{1}: I \neq \emptyset} \sum_{j=0}^{\underline{n}} \bar{n}^{r_{1}-2} \bar{n}^{r_{2}-2}(j+1)^{-(d-2) / 2} \leqslant C \bar{n}^{r-3}
$$

(with $C$ dependent on $r$ ), as required.

We estimate the term in (7.14) containing $R$ according to whether $r_{2}+1=3$ (in which case $|I|=1$ and $r_{1}=r-1$ ) or $r_{2}+1 \geqslant 4$ (in which case $|I| \geqslant 2$ ). When $r_{2}+1=3$, we write $I=\{i\}$, and we use $c^{(3)}=0$ and (7.10) to bound the term containing $R$ by

$$
C \bar{n}^{r-3} \sum_{i \in J_{1}} \sum_{j=0}^{\underline{n}} \sum_{l=1}^{\underline{n}_{J \backslash\left\langle i i^{-j}\right.}}\left(l \vee\left(n_{i}-j\right)+1\right)^{-d / 2} \leqslant C \bar{n}^{r-3},
$$


as required. When $r_{2}+1 \geqslant 4$, by (7.9)-(7.10) the term containing $R$ is bounded above by

$$
C \sum_{I \subset J_{1}:|I| \geqslant 2} \bar{n}^{r_{1}-2} \bar{n}^{r_{2}-3} \sum_{j=0}^{\underline{n}} \sum_{l=1}^{\underline{n}_{J \backslash I}-j}(l+1)^{-(d-4) / 2} \leqslant C \bar{n}^{r-4} \underline{n}_{J \backslash I}^{2-\delta} .
$$

We may again assume, as we did under (7.15), that $n_{1} \leqslant \bar{n}$, so that $\underline{n}_{J \backslash I} \leqslant n_{1} \leqslant \bar{n}$. This gives the desired bound on (7.18) and completes the proof.

\subsection{Diagrammatic bounds}

It remains to prove Lemma 7.1. The proof is in two steps, which will be carried out in this and the next section. In this section, we will show that the left sides of (7.7)-(7.11) can be bounded above by certain diagrams, as we did for $\pi(\boldsymbol{x})$ in Section 4.1. Our main conclusion will be the inequalities (7.21)-(7.25) below. In Section 7.3, we will complete the proof of Lemma 7.1 by estimating these diagrams, using methods analogous to those of Section 4.2.

Throughout this section, we fix $s \geqslant 3$ and drop the corresponding superscript from $A^{(s)}, b^{(s)}, c^{(s)}$ and $d^{(s)}$. In its place, we will instead use superscripts $N$ and $M$ to denote the order to which the expansion has been performed. We will use the $\boldsymbol{x}$ notation for vertices.

The quantities $A$ and $B$ are given by sums over $N$ of $A^{(N)}$ and $B^{(N)}$, with the latter defined by (5.5), (5.13) and (5.25)-(5.26). As was pointed out below (5.26), $B^{(N)}$ is a minor variation on $A^{(N)}$. By definition, $B^{(N)}=b^{(N)}+c^{(N)}$, with $b^{(N)}$ and $c^{(N)}$ forming a partition of the various contributions to $B^{(N)}$. Once we have obtained bounds on $A^{(N)}$, bounds on $b^{(N)}$ and $c^{(N)}$ will follow easily.

The difference between $A^{(N)}$ and $\pi^{(N)}$ is due to fact that the event $G^{\prime \prime}\left(\boldsymbol{v}_{j-1}, \boldsymbol{u}_{j}, \boldsymbol{v}_{j}, \overrightarrow{\boldsymbol{x}}_{I_{j}}\right.$; $\left.\widetilde{C}_{j-1}\right)$, rather than $E^{\prime \prime}\left(\boldsymbol{v}_{j-1}, \boldsymbol{u}_{j}, \boldsymbol{v}_{j} ; \widetilde{C}_{j-1}\right)$, occurs in (5.25), as well as to the extra connections to $\overrightarrow{\boldsymbol{x}}_{I_{0}}$ present in the expectation at level-0. As was observed in (5.17), these differences disappear at any level with $I_{j}=\emptyset$. To understand the effect of a nested expectation containing the event $G^{\prime \prime}\left(\boldsymbol{v}_{j-1}, \boldsymbol{u}_{j}, \boldsymbol{v}_{j}, \overrightarrow{\boldsymbol{x}}_{I_{j}} ; \widetilde{C}_{j-1}\right)$ with $I_{j} \neq \emptyset$, we begin by analysing this event in some detail. As in Section 4.1.1, we will need to consider the set $G^{\prime}\left(\boldsymbol{v}_{j-1}, \boldsymbol{u}_{j}, \overrightarrow{\boldsymbol{x}}_{I_{j}} ; \widetilde{\boldsymbol{C}}_{j-1}\right)$ in conjunction with the event $\left\{\boldsymbol{w}_{j+1} \in \widetilde{C}_{j}\right\}$ that arises from bounding the expectation at level- $(j+1)$.

Recall the decomposition of $G^{\prime}$ into $G_{1}^{\prime}$ and $G_{2}^{\prime}$ in (6.8)-(6.9). We will estimate the contributions due to $G_{1}^{\prime}$ and $G_{2}^{\prime}$ separately. We will use the constructions 1,2 and $E$ defined in Definitions 4.1-4.2, as well as two new constructions $\ell$ and $\widetilde{E}$ defined in Definitions 7.2-7.3. Construction $\ell$ adds a new line to a diagram, while construction $\widetilde{E}$ is a modification of construction $E$.

\subsubsection{The event $G_{1}^{\prime}$ and construction $\ell$}

We begin with the event $G_{1}^{\prime}$, as this event is closest to the event $E$, and involves estimates similar to those used to estimate $\pi$ in Section 4.1. By definition,

$$
\begin{aligned}
& G_{1}^{\prime}\left(\boldsymbol{v}_{j-1}, \boldsymbol{u}_{j}, \overrightarrow{\boldsymbol{x}}_{I_{j}} ; \widetilde{C}_{j-1}\right) \cap\left\{\boldsymbol{w}_{j+1} \in \widetilde{C}_{j}\right\} \\
& \quad \subset E^{\prime}\left(\boldsymbol{v}_{j-1}, \boldsymbol{u}_{j} ; \widetilde{C}_{j-1}\right) \cap\left\{\boldsymbol{v}_{j-1} \rightarrow \boldsymbol{w}_{j+1}\right\} \cap\left\{\boldsymbol{v}_{j-1} \rightarrow \overrightarrow{\boldsymbol{x}}_{I_{j}}\right\} .
\end{aligned}
$$


The BK inequality can be applied to estimate the expectation of the indicator of the right side in a similar fashion to the estimate (4.4), which corresponds to the case $I_{j}=\emptyset$. The difference when $I_{j}$ is nonempty entails adding additional vertices and lines to the diagram on the right side of (4.4), to make additional connections to $\overrightarrow{\boldsymbol{x}}_{I_{j}}$. These connections will be handled using the constructions defined in the following definition.

DEFINITION 7.2. - (a) Given a diagram containing the vertex $\boldsymbol{u}$, construction $\ell_{\boldsymbol{u}}(\boldsymbol{y})$ is the operation in which a line is added from $\boldsymbol{u}$ to $\boldsymbol{y}$ followed by a sum over $\boldsymbol{u}$. Explicitly, this means that we multiply the diagram by $\tau(\boldsymbol{y}-\boldsymbol{u})$ and sum over $\boldsymbol{u}$.

(b) Given a diagram and a line $\lambda$ in the diagram, construction $\ell^{\lambda}(\boldsymbol{y})$ is the diagram obtained by inserting a new vertex $\boldsymbol{u}$ on line $\lambda$, followed by construction $\ell_{\boldsymbol{u}}(\boldsymbol{y})$. Explicitly, this means that the two-point function $\tau(v-w)$ associated with line $\lambda$ is replaced by $\sum_{\boldsymbol{u}} \tau(\boldsymbol{v}-\boldsymbol{u}) \tau(\boldsymbol{u}-\boldsymbol{w}) \tau(\boldsymbol{y}-\boldsymbol{u})$. Construction $\ell(\boldsymbol{y})$ is then defined to be the procedure that applies construction $\ell^{\lambda}(\boldsymbol{y})$ to each line $\lambda$ in a given graph, and then sums the resulting diagrams over the lines $\lambda$ in the graph.

(c) Given a diagram, construction $\ell(\vec{y})$ is the sum of all diagrams obtained by first performing construction $\ell\left(\boldsymbol{y}_{1}\right)$, then performing construction $\ell\left(\boldsymbol{y}_{2}\right)$ to each of the resulting diagrams, and so on in an iterative fashion until the components of $\overrightarrow{\boldsymbol{y}}$ have been exhausted.

The probability of the right side of (7.19) is then bounded above by the result of applying construction $\ell\left(\overrightarrow{\boldsymbol{x}}_{I_{j}}\right)$ to the diagram on the right side of (4.4). This is depicted in Fig. 11. In assembling the diagrams arising from each expectation, we will again shift the connection joining $\boldsymbol{z}_{j}$ to $\boldsymbol{w}_{j+1}$ from level- $j$ to level- $(j+1)$, as in Fig. 6 . This shift is also depicted in Fig. 11. The admissible lines, on which $z_{j}$ may lie, are drawn in bold in Fig. 11. Thus the event $G_{1}^{\prime}$ has the same effect as the event $E^{\prime}$, apart from an additional construction $\ell\left(\overrightarrow{\boldsymbol{x}}_{I_{j}}\right)$. Note that the first line added in applying construction $\ell\left(\overrightarrow{\boldsymbol{x}}_{I_{j}}\right)$ can be attached at $\mathcal{O}\left(n_{1}\right)$ sites, since the time components of each of $\boldsymbol{w}_{j+1}$ and $\boldsymbol{u}_{j}$ are at most $n_{1}$. The remaining added lines can be attached at $\mathcal{O}(\bar{n})$ sites, since a point of attachment can be no later than the time coordinates of the endpoints of the attached line and of the later endpoint of the line to which it is attached.

To summarise, the event $G_{1}^{\prime}$ leads to construction $E$ followed by construction $\ell\left(\overrightarrow{\boldsymbol{x}}_{I_{j}}\right)$. This corresponds to a minor modification of the procedure used in Section 4.1 to estimate $\pi$, where construction $E$ alone was applied.
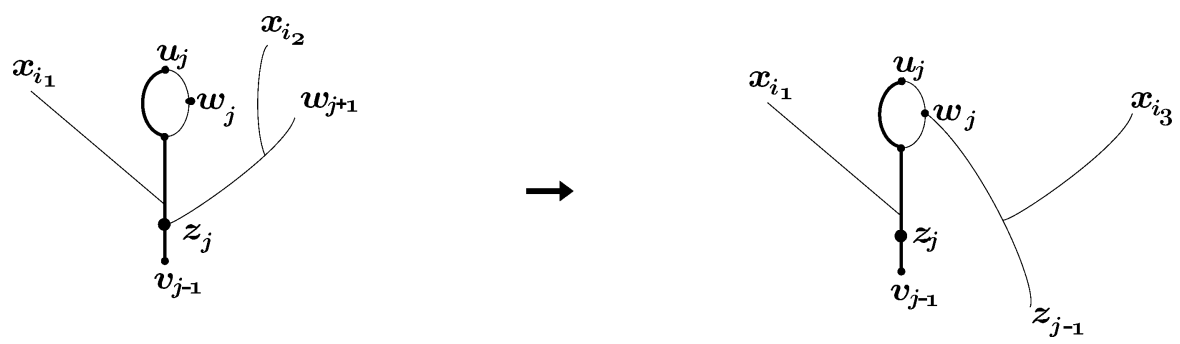

Fig. 11. A bound on the event $G_{1}^{\prime}\left(\boldsymbol{v}_{j-1}, \boldsymbol{u}_{j}, \overrightarrow{\boldsymbol{x}}_{I_{j}} ; \widetilde{C}_{j-1}\right) \cap\left\{\boldsymbol{w}_{j+1} \in \widetilde{C}_{j}\right\}$, showing also the effect of the shifted lines. The vertex $z_{j}$ must lie on a bold line. 
(a)
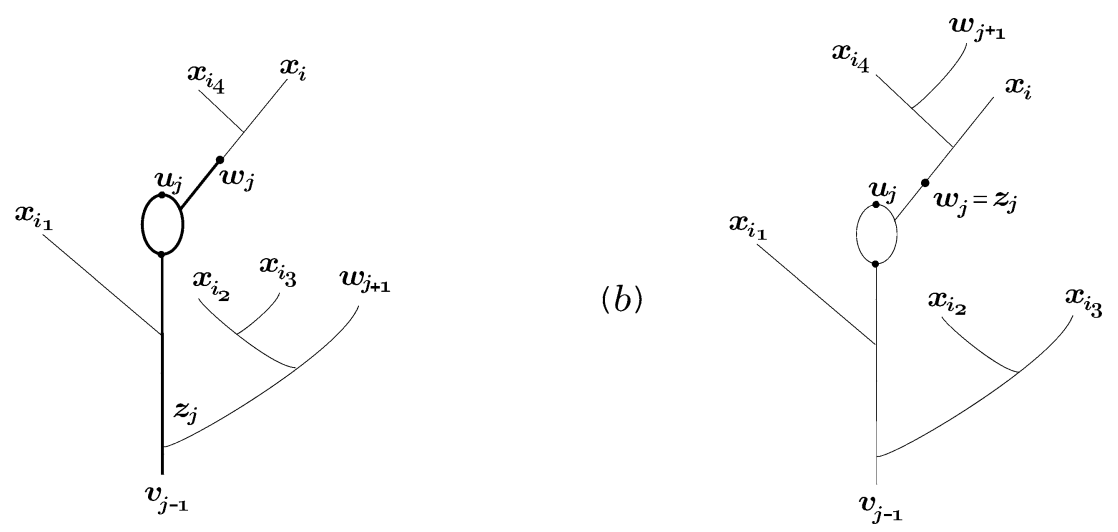

Fig. 12. Connections implied by the event $G_{2}^{\prime}\left(\boldsymbol{v}_{j-1}, \boldsymbol{u}_{j}, \overrightarrow{\boldsymbol{x}}_{I_{j}} ; \widetilde{C}_{j-1}\right) \cap\left\{\boldsymbol{w}_{j+1} \in \widetilde{C}_{j}\right\}$ when (a) $\boldsymbol{w}_{j} \not \rightarrow \boldsymbol{w}_{j+1}$, and (b) $\boldsymbol{w}_{j} \rightarrow \boldsymbol{w}_{j+1}$. In (a), the site $z_{j}$ must lie on a bold line.

\subsubsection{The event $\boldsymbol{G}_{\mathbf{2}}^{\prime}$, the function $\widetilde{\boldsymbol{P}}_{\mathbf{2}}$, and construction $\widetilde{\boldsymbol{E}}$}

Next, we consider $G_{2}^{\prime}\left(\boldsymbol{v}_{j-1}, \boldsymbol{u}_{j}, \overrightarrow{\boldsymbol{x}}_{I_{j}} ; \widetilde{C}_{j-1}\right) \cap\left\{\boldsymbol{w}_{j+1} \in \widetilde{\boldsymbol{C}}_{j}\right\}$. Again we will use diagrammatic constructions, including a new construction $\widetilde{E}$ that is a modification of construction $E$. By definition, the event $G_{2}^{\prime}\left(\boldsymbol{v}_{j-1}, \boldsymbol{u}_{j}, \overrightarrow{\boldsymbol{x}}_{I_{j}} ; \widetilde{C}_{j-1}\right) \cap\left\{\boldsymbol{w}_{j+1} \in \widetilde{C}_{j}\right\}$ is a subset of the event that (i) $\boldsymbol{v}_{j-1} \rightarrow \boldsymbol{u}_{j}$, (ii) the bottom of the last sausage for this connection is connected to an $\boldsymbol{x}_{i}$ via a site $\boldsymbol{w}_{j} \in \widetilde{C}_{j-1}$, (iii) $\boldsymbol{v}_{j-1} \rightarrow \boldsymbol{w}_{j+1}$, and (iv) $\boldsymbol{v}_{j-1} \rightarrow \overrightarrow{\boldsymbol{x}}_{I_{j} \backslash\{i\}}$. Examples of the disjoint connections implied by this are depicted schematically in Fig. 12. A site $z_{j}$ is identified as follows, according to whether or not $\boldsymbol{w}_{j} \rightarrow \boldsymbol{w}_{j+1}$. If $\boldsymbol{w}_{j} \not \boldsymbol{\boldsymbol { w } _ { j + 1 }}$, then we choose $\boldsymbol{z}_{j}$ such that $\boldsymbol{v}_{j-1} \rightarrow \boldsymbol{z}_{j}$, either $\boldsymbol{z}_{j} \rightarrow \boldsymbol{w}_{j}$ or $\boldsymbol{w}_{j} \rightarrow \boldsymbol{z}_{j}$, and $\boldsymbol{z}_{j} \rightarrow \boldsymbol{w}_{j+1}$. This is depicted in Fig. 12(a). The bold lines in Fig. 12(a) are the admissible lines, on which $\boldsymbol{z}_{j}$ must lie when $\boldsymbol{w}_{j} \not \rightarrow \boldsymbol{w}_{j+1}$. If, on the other hand, $\boldsymbol{w}_{j} \rightarrow \boldsymbol{w}_{j+1}$, then we set $\boldsymbol{z}_{j}=\boldsymbol{w}_{j}$. This is depicted in Fig. 12(b).

The line joining $\boldsymbol{z}_{j}$ to $\boldsymbol{w}_{j+1}$ (together with any branches to points $\boldsymbol{x}_{k}$ ) will be shifted to the next expectation, as in Section 4.1.1. We will distinguish the two cases (a) $\boldsymbol{w}_{j} \nrightarrow \rightarrow \boldsymbol{w}_{j+1}$ and (b) $\boldsymbol{w}_{j} \rightarrow \boldsymbol{w}_{j+1}$ depicted in Fig. 12. In case (a), a line will be present from $\boldsymbol{w}_{j}$ to the site $\boldsymbol{x}_{i}$ identified in item (ii) of the previous paragraph, but we will regard this line as to be added via a later application of construction $\ell_{\boldsymbol{w}_{j}}\left(\boldsymbol{x}_{i}\right)$. In case (b), the shifted line is required to have a branch to the site $\boldsymbol{x}_{i}$, but we will regard this branch as to be added via a later application of construction $\ell\left(\boldsymbol{x}_{i}\right)$. The procedure can be described in terms of the function $\widetilde{P}_{2}\left(\boldsymbol{u}_{j-1}, \boldsymbol{z}_{j-1}, \boldsymbol{u}_{j}, \boldsymbol{z}_{j}, \boldsymbol{w}_{j}\right)$ defined in Fig. 13. When using $\widetilde{P}_{2}\left(\boldsymbol{u}_{j-1}, \boldsymbol{z}_{j-1}, \boldsymbol{u}_{j}, \boldsymbol{z}_{j}, \boldsymbol{w}_{j}\right)$, the following rules summarise the above discussion and must be followed: (i) construction $\ell_{\boldsymbol{w}_{j}}\left(\boldsymbol{x}_{i}\right)$ must be applied to the first term, and (ii) construction $\ell\left(\boldsymbol{x}_{i}\right)$ must be applied to the line joining $\boldsymbol{w}_{j}=\boldsymbol{z}_{j}$ to $\boldsymbol{w}_{j+1}$ that was shifted from the second term. The function $\widetilde{P}_{2}\left(\boldsymbol{u}_{j-1}, \boldsymbol{z}_{j-1}, \boldsymbol{u}_{j}, \boldsymbol{z}_{j}, \boldsymbol{w}_{j}\right)$ then replaces the function $P_{2}\left(\boldsymbol{u}_{j-1}, z_{j-1}, \boldsymbol{u}_{j}, \boldsymbol{z}_{j}\right)$ of Section 4.1.1, in an expectation containing $G_{2}$. Construction $\ell\left(\overrightarrow{\boldsymbol{x}}_{I_{j} \backslash\{i\}}\right)$ must also be applied appropriately in conjunction with $\widetilde{P}_{2}$. Finally, a sum is performed over $i \in I_{j}$. We summarise the above with construction $\widetilde{E}$, defined as follows. 


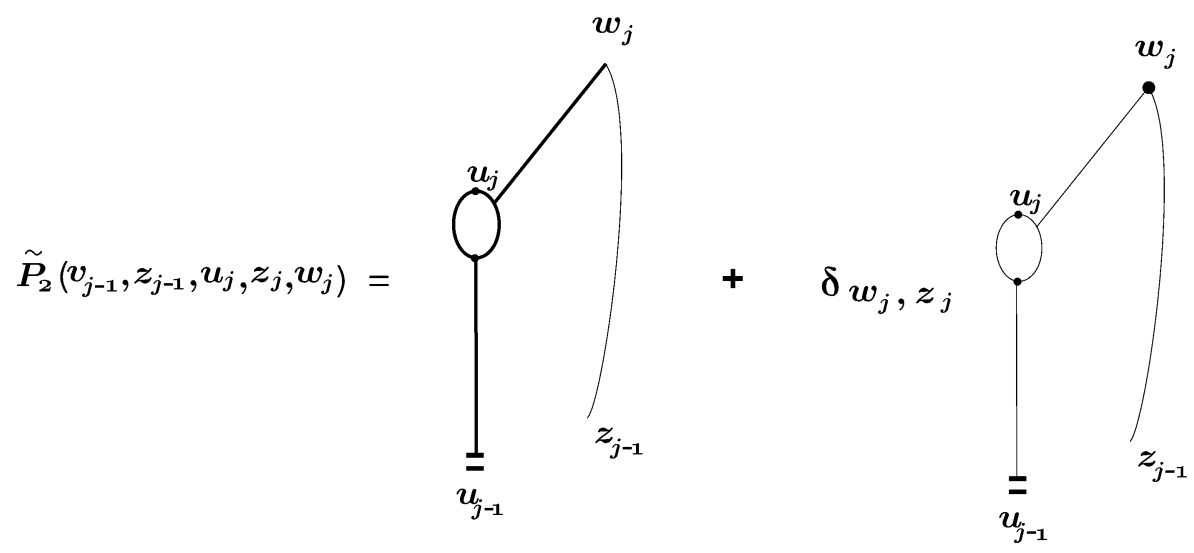

Fig. 13. Definition of $\widetilde{P}_{2}\left(\boldsymbol{u}_{j-1}, \boldsymbol{z}_{j-1}, \boldsymbol{u}_{j}, \boldsymbol{z}_{j}, \boldsymbol{w}_{j}\right)$. In the first term, a sum is implied over insertion of $z_{j}$ on any of the bold (admissible) lines.

DEFINITION 7.3. - Given a diagram containing vertex $\boldsymbol{u}_{j-1}$ and containing a certain set of admissible lines, construction $\widetilde{E}_{\boldsymbol{u}_{j-1}}\left(\boldsymbol{u}_{j}, \boldsymbol{w}_{j}\right)$ produces the new diagram defined as follows. First, construction $1\left(z_{j-1}\right)$ is performed on an admissible line. The diagram is then multiplied by

$$
\sum_{\boldsymbol{v}_{j-1}, \boldsymbol{t}, \boldsymbol{y}} p_{c} D\left(\boldsymbol{v}_{j-1}-\boldsymbol{u}_{j-1}\right) \tau\left(\boldsymbol{t}-\boldsymbol{v}_{j-1}\right) \tau\left(\boldsymbol{u}_{j}-\boldsymbol{t}\right) \tau(\boldsymbol{y}-\boldsymbol{t}) \tau\left(\boldsymbol{u}_{j}-\boldsymbol{y}\right) \tau\left(\boldsymbol{w}_{j}-\boldsymbol{y}\right) \tau\left(\boldsymbol{w}_{j}-\boldsymbol{z}_{j-1}\right)
$$

and summed over $z_{j-1}$ and over the admissible lines of the original diagram (see the first term of Fig. 13 for a depiction of (7.20)). The result is a sum of diagrams. An admissible line of a resulting diagram is any line in (7.20) except $\tau\left(\boldsymbol{w}_{j}-\boldsymbol{z}_{j-1}\right)$. With an abuse of terminology, we also include the site $\boldsymbol{w}_{j}$ itself as one of the admissible "lines". Following construction $\widetilde{E}_{\boldsymbol{u}_{j-1}}\left(\boldsymbol{u}_{j}, \boldsymbol{w}_{j}\right)$, the constructions outlined in rules (i) and (ii) above should be carried out, with rule (ii) invoked when $w_{j}$ is used as an admissible "line" in a further construction $E$ or $\widetilde{E}$. If there is no such further construction (so no further shifted line), then rule (i) applies also in this case.

For later use, we note that construction $\widetilde{E}_{\boldsymbol{u}_{j-1}}\left(\boldsymbol{u}_{j}, \boldsymbol{w}_{j}\right)$ is equivalent to application of construction $2_{\boldsymbol{u}_{j-1}}^{(1)}\left(\boldsymbol{w}_{j}\right)$, followed by construction $1(\boldsymbol{y})$ applied on the line from $\boldsymbol{v}_{j-1}$ to $\boldsymbol{w}_{j}$, followed by construction $2_{\boldsymbol{y}}^{(0)}\left(\boldsymbol{u}_{j}\right)$ with the line from $\boldsymbol{v}_{j-1}$ to $\boldsymbol{y}$ serving as the unique admissible line.

To summarise, the event $G_{2}^{\prime}$ leads to construction $\widetilde{E}$ followed by construction $\ell_{\boldsymbol{w}_{j}}\left(\boldsymbol{x}_{i}\right)$ in case (a), and $\ell\left(\boldsymbol{x}_{i}\right)$ applied to the line from $\boldsymbol{w}_{j}=\boldsymbol{z}_{j}$ in case (b), completed with construction $\ell\left(\overrightarrow{\boldsymbol{x}}_{I_{j} \backslash\{i\}}\right)$. Finally, a sum is performed over $i \in I_{j}$.

\subsubsection{Bounds on $A, b, c$}

We now define the diagrams that serve as upper bounds for $A, b, c$. Given a nonempty subset $\gamma \subset\{0, \ldots, N\}$, consider a nested expectation contributing to (5.25) in which the event $G$ appears at level- $j$ with $I_{j} \neq \varnothing$ in the expectations indexed by the set $\gamma$, and $E$ appears in the remaining expectations. In the convolution of $P_{1}, P_{2}$ and $P_{3}$ defining $P^{(N)}$ 
$(a)$

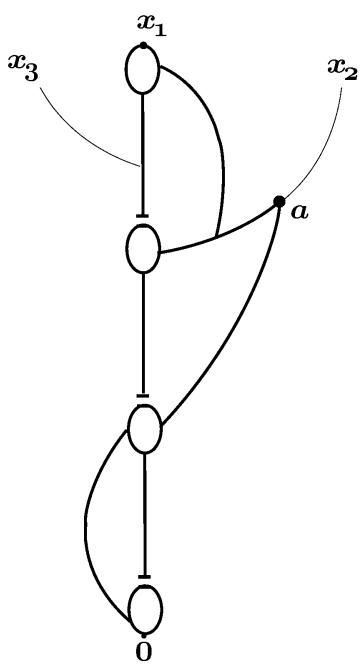

(b)

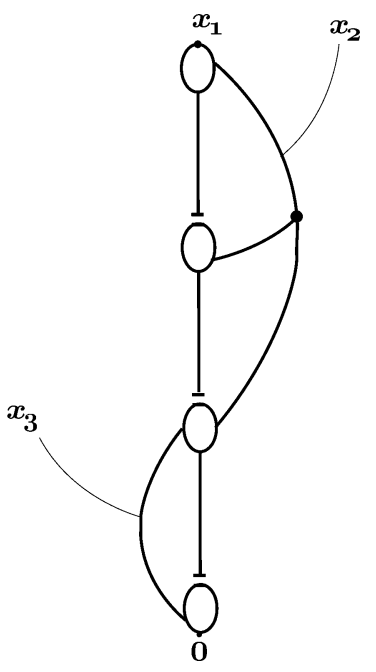

Fig. 14. Examples of diagrams contributing to $P^{(3), 1}\left(\boldsymbol{x}_{1}, \boldsymbol{x}_{2}, \boldsymbol{x}_{3}\right)$, with the lines in (a) $S^{(3)}\left(\boldsymbol{x}_{1}, \boldsymbol{a}\right)$ and (b) $S^{(3)}\left(\boldsymbol{x}_{1}\right)$ drawn in bold.

in (4.9), we replace $P_{2}$ by $P_{2}+\widetilde{P}_{2}$ at level-s for each $s \in \gamma$. (This applies for $s \neq 0, N$; levels- 0 and $N$ require minor modifications very briefly indicated below.) Starting from the bubble, any of these diagrams can be obtained by application of constructions $E$ and $\widetilde{E}$. We refer to the sum of resulting diagrams as $\sum_{\alpha \subset \gamma} S^{(N)}\left(\boldsymbol{x}_{1}, \overrightarrow{\boldsymbol{a}}_{\alpha}\right)$, where $\alpha$ denotes the subset of $\gamma$ for which the term $\widetilde{P}_{2}$ was used and for which $\boldsymbol{w}_{j}$ was not used as an admissible "line" (and hence has not been summed over), $\overrightarrow{\boldsymbol{a}}_{\alpha}$ is the set of $\boldsymbol{w}_{j}$ that have not been summed, and $S^{(N)}\left(\boldsymbol{x}_{1}, \overrightarrow{\boldsymbol{a}}_{\alpha}\right)$ represents the sum of diagrams arising with these constraints. We then apply the constructions $\ell\left(\vec{x}_{I_{j}}\right)$ to the diagrams contributing to $S^{(N)}\left(\boldsymbol{x}_{1}, \overrightarrow{\boldsymbol{a}}_{\alpha}\right)$, adding the $\boldsymbol{x}_{j}$ 's associated to each expectation to the appropriate lines, and paying attention to the rules (i) and (ii) associated with construction $\widetilde{E}$. In the special case where $I_{0}$ is nonempty, we apply construction $\ell\left(\overrightarrow{\boldsymbol{x}}_{I_{0}}\right)$ appropriately. If $I_{N}$ is nonempty, then we omit the vertex $\boldsymbol{z}_{N}$ from the level- $N$ construction. The result is a sum of diagrams which we denote by $P^{(N), j}\left(\overrightarrow{\boldsymbol{x}}_{J}\right)$, with $j=1$ arising from the case $|\gamma|=1$, and with $j=2$ arising from $|\gamma| \geqslant 2$. Examples are depicted in Fig. 14. Fig. 14(a) should be compared to Fig. 12(a) and the first term of Fig. 13, while Fig. 14(b) should be compared to Fig. 12(b) and the second term of Fig. 13.

The above discussion then implies that

$$
A^{(N)}\left(\overrightarrow{\boldsymbol{x}}_{J}\right) \leqslant P^{(N), 1}\left(\overrightarrow{\boldsymbol{x}}_{J}\right)+P^{(N), 2}\left(\overrightarrow{\boldsymbol{x}}_{J}\right) \quad(N \geqslant 0) .
$$

It then follows from the simple relation between $B^{(N)}$ and $A^{(N)}$ described under (5.26), together with the decomposition $B^{(N)}=b^{(N)}+c^{(N)}$ discussed around (6.2), that

$$
\begin{array}{ll}
b^{(N)}\left(\overrightarrow{\boldsymbol{x}}_{J}\right) \leqslant \sum_{\boldsymbol{u}} p_{c} D\left(\boldsymbol{x}_{1}-\boldsymbol{u}\right) P^{(N), 1}\left(\boldsymbol{u}, \overrightarrow{\boldsymbol{x}}_{J \backslash\{1\}}\right) & (N \geqslant 0), \\
c^{(N)}\left(\overrightarrow{\boldsymbol{x}}_{J}\right) \leqslant \sum_{\boldsymbol{u}} p_{c} D\left(\boldsymbol{x}_{1}-\boldsymbol{u}\right) P^{(N), 2}\left(\boldsymbol{u}, \overrightarrow{\boldsymbol{x}}_{J \backslash\{1\}}\right) & (N \geqslant 1) .
\end{array}
$$




\subsubsection{Bounds on $C$ and $d$}

The diagrammatic bounds on $C$ and $d$ involve estimation of the terms $C^{(N, M)}$ and $d^{(N, M)}$ defined in (6.17)-(6.20). For this, we first bound the event $H_{j}$ above by an event expressing the existence of various disjoint connections, then use Fubini's Theorem to restore the original order of the expectations, and finally use the BK inequality. The process is tedious and we simply state the results rather than including all the details.

We begin with $C^{(N, M)}\left(\boldsymbol{v}_{1}, \boldsymbol{v}_{2}\right)$. This involves a doubly nested expectation in which all expectations contain an event $E^{\prime}$, except level- $(j, 0)$ where the event $H_{j}$ appears. There are two contributions to the event $H_{j}$ defined in (6.6), corresponding to the events $G_{1}$ and $G_{2}$ of (6.8)-(6.9).

Consider first the contribution to the case $M=0$ due to $G_{1}$. In this case, $v_{2}$ is the later endpoint of a bond $\left(\boldsymbol{u}_{2}, \boldsymbol{v}_{2}\right)$ which served as a cutting bond as defined above (6.10). This contribution can be bounded above by the diagrams obtained from $\sum_{\boldsymbol{u}_{1}} P^{(N)}\left(\boldsymbol{u}_{1}\right) p_{c} D\left(\boldsymbol{v}_{1}-\boldsymbol{u}_{1}\right)$ as follows. First, for each line $\lambda$ in $P^{(N)}\left(\boldsymbol{u}_{1}\right)$, construction $1^{\lambda}(\boldsymbol{w})$ is performed. Then construction $2_{w}^{(0)}\left(\boldsymbol{u}_{2}\right)$ is applied, with a finite set of admissible lines in applying this construction. The result is then multiplied by $p_{c} D\left(\boldsymbol{v}_{2}-\boldsymbol{u}_{2}\right)$ and summed over $\boldsymbol{u}_{2}$. See Fig. 10 . When $M \geqslant 1$, the contribution due to $G_{1}$ involves application of construction $1^{\lambda}(\boldsymbol{w})$, then construction $2_{\boldsymbol{w}}^{(0)}\left(\boldsymbol{a}_{0}\right)$, then $M$ construction $E$ 's ending at the vertex $\boldsymbol{u}_{2}$, followed by multiplication by $p_{c} D\left(\boldsymbol{v}_{2}-\boldsymbol{u}_{2}\right)$ and summation over $\boldsymbol{u}_{2}$.

An upper bound for the contribution to $C^{(N, M)}\left(\boldsymbol{v}_{1}, \boldsymbol{v}_{2}\right)$ due to the contribution to $H_{j}$ from $G_{2}$ is obtained as follows. For $M=0$, the upper bound on $b^{(N)}$ applies after the following modifications. Firstly, the construction $\ell_{w}\left(\boldsymbol{x}_{2}\right)$ or $\ell\left(\boldsymbol{x}_{2}\right)$ arising from rule (i) or (ii) is replaced by a construction $2_{w}^{(0)}\left(\boldsymbol{u}_{2}\right)$ (this entails an additional construction $1(\boldsymbol{w})$ when construction $\ell\left(\boldsymbol{x}_{2}\right)$ is involved). This effectively replaces a "tail" to $\boldsymbol{x}_{2}$ by a double connection. See Fig. 10. For $M \geqslant 1$, further application of $M$ construction $E$ 's is required, in a similar fashion to the upper bound on the contribution due to $G_{1}$. Finally, an additional factor $\sum_{\boldsymbol{u}_{2}} p_{c} D\left(\boldsymbol{v}_{2}-\boldsymbol{u}_{2}\right)$ is required.

Denoting the sum of all diagrams arising as above by $Q^{(N, M)}$, we have

$$
\left|C^{(N, M)}\left(\boldsymbol{v}_{1}, \boldsymbol{v}_{2}\right)\right| \leqslant Q^{(N, M)}\left(\boldsymbol{v}_{1}, \boldsymbol{v}_{2}\right)
$$

Examples of diagrams contributing to $Q^{(3,2)}\left(\boldsymbol{v}_{1}, \boldsymbol{v}_{2}\right)$ are depicted in Fig. 15.

For the bounds on $d$, it is convenient to denote by $\widetilde{Q}^{(N, M)}\left(\boldsymbol{v}_{1}, \boldsymbol{u}_{2}\right)$ the diagrams arising in $Q^{(N, M)}\left(\boldsymbol{v}_{1}, \boldsymbol{v}_{2}\right)$ before multiplication by $p_{c} D\left(\boldsymbol{v}_{2}-\boldsymbol{u}_{2}\right)$ and summation over $\boldsymbol{u}_{2}$. For $s=3$, an upper bound for $d^{(N, M)}\left(\boldsymbol{v}, \boldsymbol{x}_{2}\right)$ is simply $\widetilde{Q}^{(N, M)}\left(\boldsymbol{v}, \boldsymbol{x}_{2}\right)$. For $s \geqslant 4$, an upper bound on $d^{(N, M)}\left(\boldsymbol{v}, \overrightarrow{\boldsymbol{x}}_{I}\right)$ is obtained by applying a construction $\ell\left(\boldsymbol{x}_{i}\right)$ to $\widetilde{Q}^{(N, M)}\left(\boldsymbol{v}, \boldsymbol{u}_{2}\right)$, then construction $\ell_{\boldsymbol{u}_{2}}\left(\boldsymbol{x}_{j}\right)$, followed by construction $\ell\left(\overrightarrow{\boldsymbol{x}}_{I \backslash\{i, j\}}\right)$ (and summation over $i, j \in I$ ). Denoting the result of these constructions by $\widetilde{Q}^{(N, M)}\left(\boldsymbol{v} ; \overrightarrow{\boldsymbol{x}}_{I}\right)$, we have

$$
d^{(N, M)}\left(\boldsymbol{v}, \overrightarrow{\boldsymbol{x}}_{I}\right) \leqslant \widetilde{Q}^{(N, M)}\left(\boldsymbol{v} ; \overrightarrow{\boldsymbol{x}}_{I}\right) \quad(s \geqslant 4) .
$$


(a)

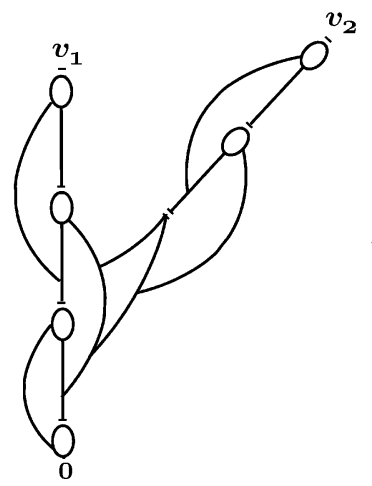

(b)

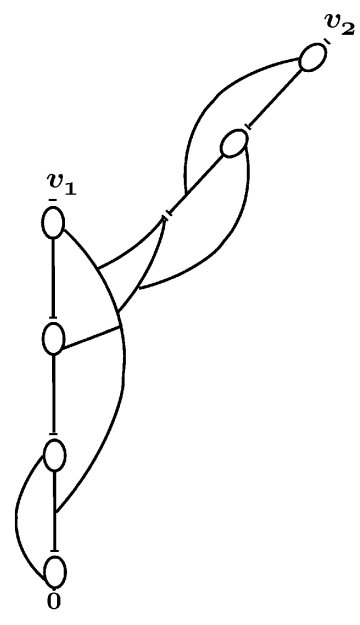

(c)

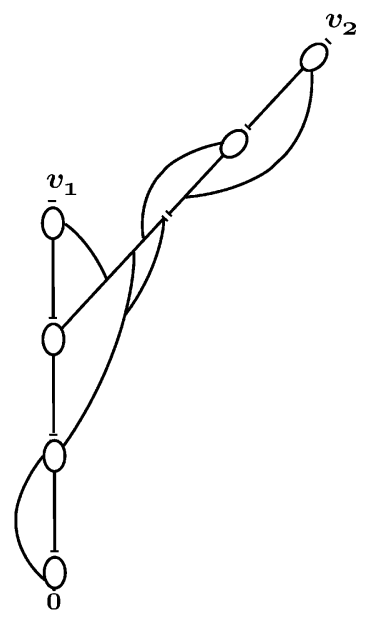

Fig. 15. Examples of diagrams contributing to $Q^{(3,2)}\left(\boldsymbol{v}_{1}, \boldsymbol{v}_{2}\right)$.

\subsection{Proof of Lemma 7.1}

We begin by showing that Lemma 7.1 is a consequence of the following lemma. We fix $s$ and omit the superscript $(s)$ from the notation. Recall that $\vec{n}_{I}$ is the vector with components $n_{i}, i \in I$.

LEMmA 7.4. - Fix $d>4, p=p_{c}, q \in\{0,2\}, s \geqslant 3, \vec{n}=\left(n_{1}, \ldots, n_{s-1}\right)$ with each $n_{i}>0$, and let $\bar{n}$ denote the second largest component of $\vec{n}$. There exist constants $L_{0}=L_{0}(d)$ and $C=C(s, d)$ such that the following bounds hold for $L \geqslant L_{0}$ and $M, N \geqslant 0$ :

$$
\begin{array}{r}
\sum_{\vec{x}_{J}} P_{\vec{n}_{J}}^{(N), j}\left(\vec{x}_{J}\right) \leqslant(C \beta)^{N} \bar{n}^{s-2-j}\left(n_{1}+1\right)^{-(d-2 j) / 2} \quad(j=1,2), \\
\sum_{x_{1}, x_{2}}\left|x_{i}\right|^{q} Q_{n_{1}, n_{2}}^{(N, M)}\left(x_{1}, x_{2}\right) \leqslant \frac{(C \beta)^{1 \vee(N+M)} \sigma^{q} n_{i}^{q / 2}}{\left(n_{1} \vee n_{2}+1\right)^{d / 2}} \quad\left(\left(n_{1}, n_{2}\right) \neq(1,1)\right), \\
\sum_{x_{1}, \vec{x}} \widetilde{Q}_{n_{1}, \vec{n}_{I}}^{(N, M)}\left(x_{1}, \vec{x}_{I}\right) \leqslant \begin{cases}(C \beta)^{N+M}\left(n_{1} \vee n_{2}+1\right)^{-d / 2} & (s=3), \\
(C \beta)^{N+M} \bar{n}^{s-4}\left(n_{1}+1\right)^{-(d-4) / 2} & (s \geqslant 4) .\end{cases}
\end{array}
$$

Proof of Lemma 7.1 assuming Lemma 7.4. - The bounds (7.7)-(7.9) on A, $b$ and $c$ follow immediately from (7.21)-(7.23) and (7.26). The bounds (7.10)-(7.11) on $d$ and $C$ follow immediately from (7.24)-(7.25) and (7.27)-(7.28).

The remainder of this section is devoted to the proof of Lemma 7.4. First we will prove (7.26) assuming that

$$
\sum_{x_{1}, \vec{a}_{\alpha}} S_{n_{1}, \vec{l}_{\alpha}}^{(N)}\left(x_{1}, \vec{a}_{\alpha}\right) \leqslant \frac{(C \beta)^{N}}{\left(n_{1} \vee l^{\prime}+1\right)^{d / 2}},
$$


where $l^{\prime}=\max _{i \in \alpha} l_{i}$ and where $S^{(N)}$ was defined in Section 7.2.3. Then we will prove (7.29). Finally, we will prove (7.27) and (7.28).

To proceed, we will need to know the effect of applying construction $\ell_{\boldsymbol{w}}(\boldsymbol{x})$ or construction $\ell(\boldsymbol{x})$ to a diagram. When construction $\ell_{\boldsymbol{w}}(\boldsymbol{x})$ is applied, followed by summation over $\boldsymbol{x}$, by Theorem 1.1(a) the resulting diagram is bounded by a constant multiple of a bound on the original diagram. By definition, construction $\ell(\boldsymbol{x})$ corresponds to an application of construction $1(\boldsymbol{u})$ followed by construction $\ell_{\boldsymbol{u}}(\boldsymbol{x})$. When followed by summation over $\boldsymbol{x}$, the effect of the latter on a diagrammatic bound is merely to multiply by a constant. By Lemma 4.6, the effect of the former on a diagrammatic bound is multiplication by $c n$, where $n$ is the minimum of the temporal component of $\boldsymbol{x}$ and the largest time coordinate of any vertex in the original diagram. (The sum over the spatial component of $\boldsymbol{u}$ has no effect, and the sum over the temporal component yields at most $c n$.) In our applications, this $n$ is at most $\bar{n}$, which is the second largest of the $n_{i}$. Thus, the net effect on a diagrammatic bound of construction $\ell(\boldsymbol{x})$ followed by summation over $\boldsymbol{x}$ is multiplication by $c n$. The constants $c$ in this discussion can depend on the number of lines in the original diagram, and hence can depend on $N$, but this dependence is polynomial and can be bounded by $C^{N}$ for some $C$.

Proof of (7.26) assuming (7.29). -

The bound on $P^{(N), 1}$. The construction of $P_{\vec{n}_{J}}^{(N), 1}\left(\vec{x}_{J}\right)$ starts from the diagrams contributing to $\sum_{\gamma:|\gamma|=1} \sum_{\alpha: \alpha \subset \gamma} \sum_{x_{1}, \vec{a}_{\alpha}} S_{n_{1}, \vec{l}_{\alpha}}^{(N)}\left(x_{1}, \vec{a}_{\alpha}\right)$. Here, $\alpha=\emptyset$ or $|\alpha|=1$.

When $\alpha=\emptyset$, construction $\ell\left(\overrightarrow{\boldsymbol{x}}_{J_{1}}\right)$ is applied to $\sum_{x_{1}} S_{n_{1}}^{(N)}\left(x_{1}\right)$. Assuming (7.29), the latter is bounded above by $(C \beta)^{N}\left(n_{1}+1\right)^{-d / 2}$. The effect of the first construction $\ell\left(\boldsymbol{x}_{i}\right)$ is to add to the estimate a factor $c\left(n_{1}+1\right)$, while each of the remaining $s-3$ produces a factor $c \bar{n}$. Altogether, this produces a factor $\bar{n}^{s-3}\left(n_{1}+1\right)$. Combined with $(C \beta)^{N}\left(n_{1}+1\right)^{-d / 2}$, this agrees with (7.26). As observed above, there are $N$-dependent factors arising from the constructions $\ell\left(\boldsymbol{x}_{i}\right)$, but these can be absorbed into the factor $C^{N}$ of (7.29).

When $|\alpha|=1$, one construction $\ell_{(a, l)}\left(\boldsymbol{x}_{i}\right)$ is applied, followed by $s-3$ applications of construction $\ell\left(\boldsymbol{x}_{j}\right)$. These constructions are applied to $\sum_{x_{1}, a} S_{n_{1}, l}^{(N)}\left(x_{1}, a\right)$, which is assumed to be bounded above by $(C \beta)^{N}\left(n_{1} \vee l+1\right)^{-d / 2}$. The time variable $l$ must be summed out after application of construction $\ell_{(a, l)}\left(\boldsymbol{x}_{1}\right)$, giving rise to a bound

$$
\sum_{l=0}^{\infty} \frac{(C \beta)^{N}}{\left(n_{1} \vee l+1\right)^{d / 2}} \leqslant \frac{(C \beta)^{N}}{\left(n_{1}+1\right)^{(d-2) / 2}}
$$

The effect of the remaining $s-3$ applications of construction $\ell\left(\boldsymbol{x}_{j}\right)$ is an additional factor $\bar{n}^{s-3}$. This gives the bound of (7.26) in this case.

The bound on $P^{(N), 2}$. When $|\gamma| \geqslant 2,|\alpha|$ may take on any value in $\{0, \ldots, N\}$, since events $G_{1}$ do not contribute to $\alpha$ and since it is possible that any $\boldsymbol{w}_{j}$ appearing due to events $G_{2}$ at levels-0 to $(N-1)$ may be used as admissible vertices at the next level, removing them from the list $\overrightarrow{\boldsymbol{a}}_{\alpha}$.

Consider first the case $|\alpha| \geqslant 2$. In this case, we proceed as above, applying construction $\ell_{\boldsymbol{a}}\left(\boldsymbol{x}_{i}\right)|\alpha|$ times (once for each component $\boldsymbol{a}$ of $\overrightarrow{\boldsymbol{a}}_{\alpha}$ ), followed by $s-2-|\alpha|$ 
applications of construction $\ell\left(\boldsymbol{x}_{j}\right)$. For the applications of construction $\ell_{\boldsymbol{a}}\left(\boldsymbol{x}_{i}\right)$, we choose the two largest time components from among these $\boldsymbol{x}_{i}$ 's, say $n_{i_{1}} \geqslant n_{i_{2}}$, and we apply these two constructions $\ell_{\boldsymbol{a}}\left(\boldsymbol{x}_{i_{j}}\right)$ first. Let $l_{\alpha}$ denote the temporal component of $\boldsymbol{a}_{\alpha}$ and let $l_{i}^{\prime}=\max _{k \in \alpha \backslash\{i\}} l_{k}$ and $l_{i, j}^{\prime}=\max _{k \in \alpha \backslash\{i, j\}} l_{k}$. Assuming (7.29), and using our observations above regarding the effect of construction $\ell_{\boldsymbol{a}}(\boldsymbol{x})$, the application of construction $\ell_{\boldsymbol{a}}\left(\boldsymbol{x}_{i_{1}}\right)$ leads to an upper bound

$$
\sum_{l_{i_{1}}=0}^{\infty} \frac{(C \beta)^{N}}{\left(n_{1} \vee l_{i_{1}}^{\prime} \vee l_{i_{1}}+1\right)^{d / 2}} \leqslant \frac{(C \beta)^{N}}{\left(n_{1} \vee l_{i_{1}}^{\prime}+1\right)^{(d-2) / 2}}
$$

Similarly, the application of construction $\ell_{\boldsymbol{a}}\left(\boldsymbol{x}_{i_{2}}\right)$ produces an upper bound

$$
\frac{(C \beta)^{N}}{\left(n_{1} \vee l_{i_{1}, i_{2}}^{\prime}+1\right)^{(d-4) / 2}} \leqslant \frac{(C \beta)^{N}}{\left(n_{1}+1\right)^{(d-4) / 2}} .
$$

The remaining $|\alpha|-2$ applications of construction $\ell_{\boldsymbol{a}}\left(\boldsymbol{x}_{i}\right)$ each give a factor of at most $\bar{n}$ from the summation over the time variable of $\boldsymbol{a}_{\alpha}$ (which is the number of possible temporal locations of $\boldsymbol{a}_{\alpha}-$ it is to avoid producing a factor $\max _{i} n_{i}$ here that we dealt with $n_{i_{1}}$ and $n_{i_{2}}$ above). Finally, the $s-2-|\alpha|$ applications of construction $\ell\left(\boldsymbol{x}_{j}\right)$ each produce an additional factor $\bar{n}$. The result is a bound

$$
\bar{n}^{s-2-|\alpha|} \bar{n}^{|\alpha|-2} \frac{(C \beta)^{N}}{\left(n_{1}+1\right)^{(d-4) / 2}},
$$

in agreement with (7.26).

This leaves the cases $|\alpha|=0,1$. In these cases, we first perform $2-|\alpha|$ constructions $1\left(\boldsymbol{a}_{i}\right)$ on diagram lines belonging to expectations that do not contain any $\boldsymbol{a}_{\alpha}$ (so no restriction when $\alpha$ is empty). This gives a factor $\mathcal{O}\left(n_{1}+1\right)^{2-|\alpha|}$, as this is the number of temporal locations at which the vertices $\boldsymbol{a}_{i}$ may be placed. We then perform 2 constructions $\ell_{\boldsymbol{a}}\left(\boldsymbol{x}_{i}\right)$ at the sites $\boldsymbol{a}_{\alpha}$ and $\boldsymbol{a}_{i}$ (so only at the two sites $\boldsymbol{a}_{i}$ when $\alpha$ is empty). Assuming (7.29) and proceeding as in (7.31)-(7.32), this leads to an upper bound, at this stage, of

$$
\left(n_{1}+1\right)^{2-|\alpha|} \frac{(C \beta)^{N}}{\left(n_{1}+1\right)^{(d-2|\alpha|) / 2}}=\frac{(C \beta)^{N}}{\left(n_{1}+1\right)^{(d-4) / 2}} .
$$

Next, we perform $s-4$ constructions $\ell\left(\boldsymbol{x}_{j}\right)$, producing an additional factor $\bar{n}^{s-4}$. This again gives a bound of the form (7.26).

Proof of (7.29). - We will prove (7.29) by induction on $N$. The induction hypothesis is that

$$
\sum_{x_{1}, \vec{a}_{\alpha}} S_{n_{1}, \vec{l}_{\alpha}}^{(N-1)}\left(x_{1}, \vec{a}_{\alpha}\right) \leqslant \frac{(C \beta)^{N-1}}{\left(n_{1} \vee l^{\prime}+1\right)^{d / 2}},
$$

with $l^{\prime}=\max _{i \in \alpha} l_{i}$. We also assume, as part of the induction hypothesis, that the bound (7.35) is obtained by applying $l^{\infty}$ and $l^{1}$ norms to diagram lines. The induction is started by noting that $S^{(0)}$ is the bubble diagram with $\alpha=\emptyset$, from which (7.35) follows from 
Theorem 1.1(a, c) by applying the $l^{\infty}$ norm to one line of the bubble and the $l^{1}$ norm to the other.

To advance the induction, we note that in constructing the diagrams $S^{(N)}$ from $S^{(N-1)}$, we apply either construction $E$ or $\widetilde{E}$ to $S^{(N-1)}$. When $S^{(N-1)}$ had its last level added via construction $\widetilde{E}$, there are two cases according to whether the next construction uses an admissible line or the admissible vertex at the level- $(N-1)$; see Fig. 13.

We first consider the case where construction $E$ is applied to level- $(N-1)$, using an admissible line rather than an admissible vertex from level- $(N-1)$. Construction $E$ is defined by (4.16) and (4.14). From (4.14), we conclude that it suffices to show that constructions $2^{(i)}$ inductively preserve the bound (7.35), provided a factor $\beta^{i}$ is produced. For this, we argue in a similar fashion to the proof of Lemma 4.6(b). Application of construction $2_{(y, m)}^{(0)}\left(x_{1}, n_{1}\right)$ to $\sum_{\vec{a}_{\alpha}} S_{m, \vec{l}_{\alpha}}^{(N-1)}\left(y, \vec{a}_{\alpha}\right)$ gives an upper bound

$$
\sum_{m=0}^{n_{1}} \sum_{j=0}^{n_{1} \wedge\left(m \vee l^{\prime}\right)} \frac{(C \beta)^{N-1}}{\left(m \vee l^{\prime}+1\right)^{d / 2}} \frac{C}{\left(n_{1}-(m \wedge j)+1\right)^{d / 2}} .
$$

Here, we have associated the $l^{\infty}$ norm to the longest of the two lines ending at $\left(x_{1}, n_{1}\right)$ that were added by construction $2^{(0)}$, and associated the $l^{1}$ norm to the other. The upper bound $n_{1} \wedge\left(m \vee l^{\prime}\right)$ occurs for the sum over $j$ for the following reasons. Firstly, $j \leqslant n_{1}$ by definition of construction $2^{(0)}$. Secondly, if an admissible line from an $\widetilde{E}$ at level$(N-1)$ is used then $j \leqslant l^{\prime}$ if $l^{\prime} \geqslant m$ and otherwise $j \leqslant m$, and $j \leqslant m$ if an admissible line from an $E$ at level- $(N-1)$ is used.

Since $\left(m \vee l^{\prime}\right)+\left(n_{1}-(m \wedge j)\right) \geqslant n_{1} \vee l^{\prime},(7.36)$ is bounded above by

$$
\frac{2^{d / 2} C^{N} \beta^{N-1}}{\left(n_{1} \vee l^{\prime}+1\right)^{d / 2}} \sum_{m=0}^{n_{1}} \sum_{j=0}^{n_{1} \wedge\left(m \vee l^{\prime}\right)}\left[\frac{1}{\left(m \vee l^{\prime}+1\right)^{d / 2}}+\frac{1}{\left(n_{1}-(m \wedge j)+1\right)^{d / 2}}\right] .
$$

For the first term in (7.37), we divide the sum according to whether $j \leqslant m$ or $j>m$. When $j \leqslant m$, the sum is bounded above by $\sum_{m=0}^{\infty} \sum_{j=0}^{m}(m+1)^{-d / 2}<\infty$. When $j>m$ we use $m \leqslant j \leqslant l^{\prime}$ to bound the sum by $\left(l^{\prime}+1\right)^{-d / 2} \sum_{j, m=0}^{l^{\prime}} 1 \leqslant\left(l^{\prime}+1\right)^{-(d-4) / 2} \leqslant 1$. The second term in (7.37) can be bounded using $\sum_{j, m=0}^{\infty}(m \vee j+1)^{-d / 2}<\infty$. This shows that construction $2^{(0)}$ inductively preserves the bound (7.35), apart from a missing factor $\beta$. Similarly, construction $2^{(1)}$ inductively preserves (7.35), but here it can be checked that a factor $\beta$ will be produced (along the lines of the discussion below (4.32)). Thus we have advanced the induction in this case.

Now suppose, on the other hand, that construction $E$ is applied to level- $(N-1)$, that there is an admissible vertex at level- $(N-1)$, and that construction $E$ makes use of this admissible vertex. Again, we use the fact that construction $E$ is given by construction $2^{(1)}$ followed by construction $2^{(0)}$. Recall the definition of $l_{i}^{\prime}$ above (7.31). Letting $l_{i}$ denote the time coordinate of the admissible vertex where construction $E$ is applied, we obtain the upper bound

$$
\sum_{m=0}^{n_{1}} \sum_{l_{i}=0}^{n_{1}} \frac{(C \beta)^{N-1}}{\left(m \vee l_{i}^{\prime} \vee l_{i}+1\right)^{d / 2}} \frac{C \beta}{\left(n_{1}-\left(m \wedge l_{i}\right)+1\right)^{d / 2}}
$$


for an application of construction $2^{(1)}$ (the additional factor $\beta$ on the right side is as discussed in the previous paragraph). By considering separately the cases $m \leqslant l_{i}$ and $l_{i} \leqslant m,(7.38)$ with the factor $(C \beta)^{N}$ omitted is bounded above by

$$
\begin{aligned}
& \sum_{0 \leqslant m \leqslant l_{i} \leqslant n_{1}} \frac{1}{\left(l_{i}^{\prime} \vee l_{i}+1\right)^{d / 2}} \frac{1}{\left(n_{1}-m+1\right)^{d / 2}} \\
& \quad+\sum_{0 \leqslant l_{i} \leqslant m \leqslant n_{1}} \frac{1}{\left(m \vee l_{i}^{\prime}+1\right)^{d / 2}} \frac{1}{\left(n_{1}-l_{i}+1\right)^{d / 2}} .
\end{aligned}
$$

The two terms in (7.39) are identical, so we consider only the second term. When $l_{i}^{\prime} \leqslant n_{1}$, the second term can be bounded by

$$
\sum_{0 \leqslant l_{i} \leqslant m \leqslant n_{1}} \frac{1}{(m+1)^{d / 2}} \frac{1}{\left(n_{1}-l_{i}+1\right)^{d / 2}} \leqslant \frac{C}{\left(n_{1}+1\right)^{d / 2}}=\frac{C}{\left(n_{1} \vee l_{i}^{\prime}+1\right)^{d / 2}},
$$

using (4.26). On the other hand, when $n_{1} \leqslant l_{i}^{\prime}$, we have instead the upper bound

$$
\frac{1}{\left(l_{i}^{\prime}+1\right)^{d / 2}} \sum_{0 \leqslant l_{i} \leqslant m \leqslant n_{1}} \frac{1}{\left(n_{1}-l_{i}+1\right)^{d / 2}} \leqslant \frac{C}{\left(l_{i}^{\prime}+1\right)^{d / 2}}=\frac{C}{\left(n_{1} \vee l_{i}^{\prime}+1\right)^{d / 2}} .
$$

This gives a bound of the desired form. Finally, application of construction $2^{(0)}$ preserves the bound, as in the previous case. Note that the upper bound on $S^{(N)}$ is in terms of a set $\alpha$ that has been reduced in size by one element from the $\alpha$ for $S^{(N-1)}$, as is appropriate in this case.

Next, we advance the inductive bound in the case where construction $\widetilde{E}$ is applied to a diagram in $S_{m, \vec{l}_{\alpha}}^{(N-1)}\left(y, \vec{a}_{\alpha}\right)$. As was pointed out below Definition 7.3, construction $\widetilde{E}_{\boldsymbol{y}}\left(\boldsymbol{x}_{1}, \boldsymbol{a}\right)$ is equivalent to application of construction $2_{\boldsymbol{y}}^{(1)}(\boldsymbol{a})$ (where $\boldsymbol{a}$ is the new vertex in $\alpha$ for level- $N$ ), followed by construction 1(t) applied on the line from $\boldsymbol{y}^{\prime}$ (the upper vertex on the pivotal bond from $\boldsymbol{y}$ ) to $\boldsymbol{a}$, followed by construction $2_{t}^{(0)}\left(\boldsymbol{x}_{1}\right)$ with the line from $y^{\prime}$ to $t$ serving as the unique admissible line. The effect of the construction $2_{y}^{(1)}(\boldsymbol{a})$ is identical to the effect of construction $E$ explained above, and produces the inductive bound produced by construction $E$. With summation over space but not time, construction $1(t)$ then produces a diagram that obeys the inductive bound with the set $\alpha$ enhanced to include $\boldsymbol{t}$. But then construction $2_{t}^{(0)}\left(\boldsymbol{x}_{1}\right)$ is again identical in its effect to the application of construction $E$ discussed above, except no additional factor of $\beta$ occurs. This completes the advancement of the induction for construction $\widetilde{E}$, and hence completes the advancement of the induction. This completes the proof of (7.29).

Proof of (7.27). - We discuss only $q=0$, since $q=2$ can be handled as in the proof of Lemma 4.5. The diagrams $Q^{(N, M)}$ were defined in Section 7.2.4. There are two contributions to $Q^{(N, M)}$, depending on whether $G_{1}$ or $G_{2}$ is used at level- $(N, 0)$. When $G_{1}$ is used, the diagrams are produced from $P_{n_{1}}^{(N)}\left(x_{1}\right)$ as described in Section 7.2.4, by first applying construction 1 , which effectively changes the bound from $(C \beta)^{N}\left(n_{1}+1\right)^{-d / 2}$ to $(C \beta)^{N}\left(n_{1} \vee n_{2}+1\right)^{-d / 2}$, and then applying construction $2^{(0)}$, followed by $M$ applications of construction $E$. The inductive proof of (7.35) shows that these last constructions do 
not affect the bound, apart from producing the necessary factor $(C \beta)^{M}$, as in (7.37)(7.38).

When $G_{2}$ is used, consider first the case where construction $\ell_{\boldsymbol{w}_{j}}\left(\boldsymbol{x}_{2}\right)$ is replaced by construction $2^{(0)}$ as explained above (7.24). In this case, the removal of the line due to construction $\ell_{\boldsymbol{w}_{j}}\left(\boldsymbol{x}_{2}\right)$ produces a contribution to $S^{(N)}\left(\boldsymbol{x}_{1}, \boldsymbol{a}_{1}\right)$, which obeys the bound of (7.35). Subsequent applications of construction $2^{(0)}$ and construction $E$ then lead to the desired bound, as in the proof of (7.26). This leaves the case where construction $\ell\left(\boldsymbol{x}_{2}\right)$ is replaced by a combination of constructions 1 and 2 , which is bounded exactly as in the discussion of $G_{1}$ above.

When $M=N=0$ and $\left(n_{1}, n_{2}\right) \neq(1,1)$, at least one diagram line is more than a single vertex, and this can easily be used to extract a factor $\beta$.

Proof of (7.28). - The omission of multiplication by $p_{c} D\left(\boldsymbol{v}_{2}-\boldsymbol{u}_{2}\right)$ has no significant effect on the bounds. For $s=3$, the desired result follows from (7.27). For $s \geqslant 4$, we begin with the bound $(C \beta)^{N+M}\left(n_{1} \vee m_{2}+1\right)^{-d / 2}$ on $\sum_{x_{1}, u_{2}} \widetilde{Q}_{n_{1}, m_{2}}^{(N, M)}\left(x_{1}, u_{2}\right)$. Application of construction $\ell\left(\boldsymbol{x}_{i}\right)$ gives rise to an additional factor $\left(n_{1} \vee m_{2}+1\right)$. Application of construction $\ell_{\boldsymbol{u}_{2}}\left(\boldsymbol{x}_{j}\right)$ gives rise to the bound

$$
\sum_{m_{2}=0}^{\infty} \frac{(C \beta)^{N+M}}{\left(n_{1} \vee m_{2}+1\right)^{(d-2) / 2}} \leqslant \frac{(C \beta)^{N+M}}{\left(n_{1}+1\right)^{(d-4) / 2}}
$$

Finally, the remaining $s-4$ applications of construction $\ell\left(\boldsymbol{x}_{k}\right)$ produce a factor $\bar{n}^{s-4}$, which gives the desired bound (7.28).

\section{Acknowledgements}

We extend sincere thanks to Takashi Hara for his significant involvement in part of this work, and, in particular, for important contributions to the first proof of our main results, using the magnetic field methods of [22] in place of the methods of Sections 5 and 6. We are also grateful to Eric Derbez for his involvement during the early stages of the project.

The work of GS was supported in part by NSERC of Canada. The work of both authors was carried out in part at Microsoft Research, the Fields Institute and McMaster University. We thank Achim Klenke, Jean-François Le Gall, Ed Perkins and Akira Sakai for useful conversations.

\section{REFERENCES}

[1] R.J. Adler, Superprocess local and intersection local times and their corresponding particle pictures, in: E. Çinlar, K.L. Chung, M.J. Sharpe (Eds.), Seminar on Stochastic Processes 1992, Birkhäuser, Boston, 1993, pp. 1-42.

[2] M. Aizenman, D.J. Barsky, Sharpness of the phase transition in percolation models, Comm. Math. Phys. 108 (1987) 489-526.

[3] M. Aizenman, C.M. Newman, Tree graph inequalities and critical behavior in percolation models, J. Stat. Phys. 36 (1984) 107-143. 
[4] D. Aldous, Tree-based models for random distribution of mass, J. Stat. Phys. 73 (1993) 625-641.

[5] M.T. Barlow, E.A. Perkins, On the filtration of historical Brownian motion, Ann. Probab. 22 (1994) 1273-1294.

[6] C. Bezuidenhout, G. Grimmett, The critical contact process dies out, Ann. Probab. 18 (1990) 1462-1482.

[7] E. Bolthausen, C. Ritzmann, A central limit theorem for convolution equations and weakly self-avoiding walks, Ann. Probab., to appear.

[8] D.C. Brydges, T. Spencer, Self-avoiding walk in 5 or more dimensions, Comm. Math. Phys. 97 (1985) 125-148.

[9] J.T. Cox, R. Durrett, E.A. Perkins, Rescaled voter models converge to super-Brownian motion, Ann. Probab. 28 (2000) 185-234.

[10] T. Cox, R. Durrett, E.A. Perkins, Rescaled particle systems converging to super-Brownian motion, in: M. Bramson, R. Durrett (Eds.), Perplexing Problems in Probability: Festschrift in Honor of Harry Kesten, Birkhäuser, Basel, 1999.

[11] D.A. Dawson, Measure-Valued Markov Processes, in: Ecole d'Eté de Probabilités de SaintFlour 1991, in: Lecture Notes in Mathematics, Vol. 1541, Springer, Berlin, 1993.

[12] E. Derbez, G. Slade, Lattice trees and super-Brownian motion, Canad. Math. Bull. 40 (1997) 19-38.

[13] E. Derbez, G. Slade, The scaling limit of lattice trees in high dimensions, Comm. Math. Phys. 193 (1998) 69-104.

[14] R. Durrett, E.A. Perkins, Rescaled contact processes converge to super-Brownian motion in two or more dimensions, Probab. Theory Related Fields 114 (1999) 309-399.

[15] E.B. Dynkin, Representation for functionals of superprocesses by multiple stochastic integrals, with applications to self-intersection local times, Astérisque 157-158 (1988) $147-171$.

[16] E.B. Dynkin, An Introduction to Branching Measure-Valued Processes, American Mathematical Society, Providence, RI, 1994.

[17] A.M. Etheridge, An Introduction to Superprocesses, American Mathematical Society, Providence, RI, 2000.

[18] G. Grimmett, Percolation, 2nd Edition, Springer, Berlin, 1999.

[19] G. Grimmett, P. Hiemer, Directed percolation and random walk, in: V. Sidoravicius (Ed.), In and Out of Equilibrium, Birkhäuser, Boston, 2002, pp. 273-297.

[20] T. Hara, G. Slade, Mean-field critical behaviour for percolation in high dimensions, Comm. Math. Phys. 128 (1990) 333-391.

[21] T. Hara, G. Slade, The number and size of branched polymers in high dimensions, J. Stat. Phys. 67 (1992) 1009-1038.

[22] T. Hara, G. Slade, The scaling limit of the incipient infinite cluster in high-dimensional percolation. I. Critical exponents, J. Stat. Phys. 99 (2000) 1075-1168.

[23] T. Hara, G. Slade, The scaling limit of the incipient infinite cluster in high-dimensional percolation. II. Integrated super-Brownian excursion, J. Math. Phys. 41 (2000) 1244-1293.

[24] R. van der Hofstad, F. den Hollander, G. Slade, Construction of the incipient infinite cluster for spread-out oriented percolation above $4+1$ dimensions, Comm. Math. Phys. 231 (2002) 435-461.

[25] R. van der Hofstad, F. den Hollander, G. Slade, A new inductive approach to the lace expansion for self-avoiding walks, Probab. Theory Related Fields 111 (1998) 253-286.

[26] R. van der Hofstad, G. Slade, The lace expansion on a tree with application to networks of self-avoiding walks, Adv. Appl. Math., to appear. 
[27] R. van der Hofstad, G. Slade, A generalised inductive approach to the lace expansion, Probab. Theory Related Fields 122 (2002) 389-430.

[28] J.-F. Le Gall, Spatial Branching Processes, Random Snakes, and Partial Differential Equations, Birkhäuser, Basel, 1999.

[29] N. Madras, G. Slade, The Self-Avoiding Walk, Birkhäuser, Boston, 1993.

[30] M.V. Menshikov, Coincidence of critical points in percolation problems, Soviet Math. Dokl. 33 (1986) 856-859.

[31] B.G. Nguyen, W.-S. Yang, Triangle condition for oriented percolation in high dimensions, Ann. Probab. 21 (1993) 1809-1844.

[32] B.G. Nguyen, W.S. Yang, Gaussian limit for critical oriented percolation in high dimensions, J. Stat. Phys. 78 (1995) 841-876.

[33] S.P. Obukhov, The problem of directed percolation, Phys. 101A (1980) 145-155.

[34] E. Perkins, Dawson-Watanabe superprocesses and measure-valued diffusions, in: P.L. Bernard (Ed.), Lectures on Probability Theory and Statistics. Ecole d'Eté de Probabilités de Saint-Flour XXIX-1999, in: Lecture Notes in Mathematics, Vol. 1781, Springer, Berlin, 2002, pp. 125-329.

[35] A. Sakai, Mean-field critical behavior for the contact process, J. Stat. Phys. 104 (2001) 111-143.

[36] A. Sakai, Hyperscaling inequalities for the contact process and oriented percolation, J. Stat. Phys. 106 (2002) 201-211.

[37] G. Slade, Lattice trees, percolation and super-Brownian motion, in: M. Bramson, R. Durrett (Eds.), Perplexing Problems in Probability: Festschrift in Honor of Harry Kesten, Birkhäuser, Basel, 1999.

[38] G. Slade, Scaling limits and super-Brownian motion, Notices Amer. Math. Soc. 49 (9) (2002) 1056-1067. 